UNITED STATES DEPARTMENT OF THE INTERIOR GEOLOGICAL SURVEY

MASSACHUSETTS HYDROLOGIC-DATA REPORT No. 14

\title{
HYDROLOGIC DATA OF THE NEPONSET AND WEYMOUTH RIVER BASINS, MASSACHUSETTS
}

BY

R. A. BRACKLEY, WILLIAM B. FLECK AND RICHARD E. WILLEY

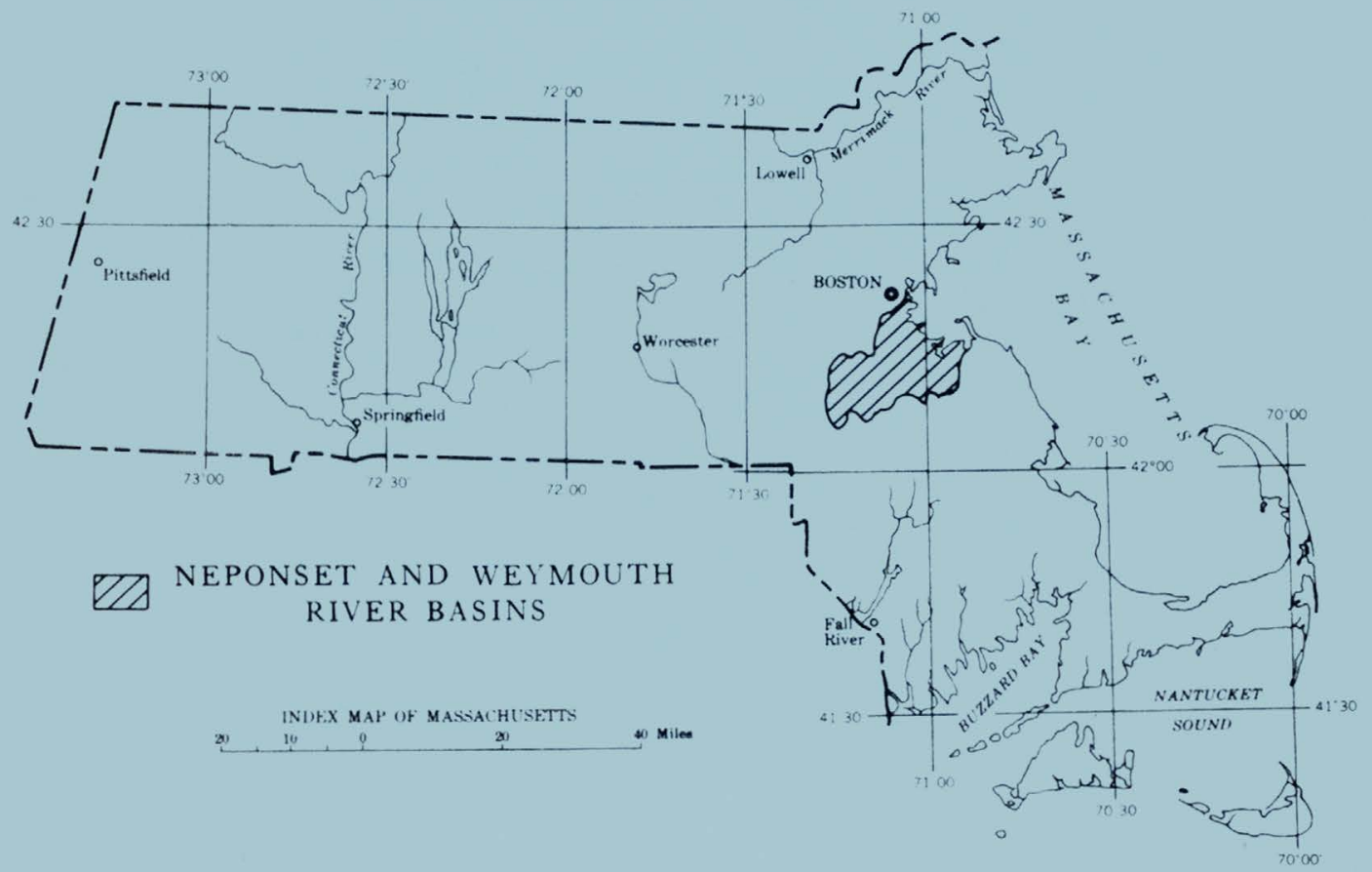

PREPARED IN COOPERATION WITH

THE COMMONWEALTH OF MASSACHUSETTS

WATER RESOURCES COMMISSION 


\section{UNITED STATES \\ DEPARTMENT OF THE INTERIOR \\ Geological Survey}

HYDROLOGIC DATA OF THE NEPONSET AND
WEYMOUTH RIVER BASINS, MASSACHUSETTS

By

R. A. Brackley, William B. Fleck, and Richard E. Willey

Massachusetts Hydrologic-Data Report No. 14

Records of surface-water discharges, selected wells and borings, and chemical analyses of water in the

Neponset and Weymouth River basins, Massachusetts

Prepared in cooperation with

THE COMMONWEALTH OF MASSACHUSETTS, WATER RESOURCES COMMISSION

Boston, Massachusetts

1973

OPEN-FILE REPORT 

Sources of information-_-

Definition of terms-_-

Selected equivalents-_-

Selected references-_-_____

ILLUSTRATIONS

Plate is in pocket

Plate 1. Map of the Neponset and Weymouth River basins, showing sites of hydrologic data.

Figure 1. Monthly mean discharge at stream-gaging stations and monthend water level in selected wells in the Neponset and Weymouth River basins, 1965-68-.--.--

\section{TABLES}

Table 1. Description of selected wells and borings-----

2. Logs of selected wells and borings-_-

3. Chemical analyses of ground water-

4. Stream sites and discharge measurements-_-_-

5. Chemical analyses of surface water-...-

6. List of basic-data reports for Massachusetts and 
, 
HYDROLOGIC DATA OF THE NEPONSET AND

WEYMOUTH RIVER BASINS, MASSACHUSETTS

By

R. A. Brackley, William B. Fleck, and Richard E. Willey

INTRODUCTION

The Neponset, Weymouth Fore, and Weymouth Back River basins occupy an area of 183 square miles in eastern Massachusetts south of Boston and Braintree, Brockton, Canton, Dedham, Dover, Foxborough, Hingham, Holbrook, Medfield, Milton, Norwood, Quincy, Randolph, Rockland, Sharon, Stoughton, Walpole, Westwood, and Weymouth.

Hydrologic data presented in this report were collected during an investigation of the water resources in the areas of these basins that are upstream from tide effect or heavy urbanization. This investigation was conducted by the U.S. Geological Survey in cooperation with the Massachusetts Water Resources Commission. The data are released in order to make available to the public basic hydrologic and related information that will facilitate the planning of water-resources development and will complement an interpretive report, "Hydrology and water resources of the Neponset and Weymouth River basins, Massachusetts" (HA-484).

The well and boring data contained herein were selected from a larger group of data in order to minimize redundancy of information for intensely drilled areas. All of the data are on file and available for inspection at the office of the U.S. Geological Survey, Water Resources Division, Boston, Massachusetts. 


\section{SOURCES OF INFORMATION}

In addition to streamflow, geologic, ground water, and chemical water-quality data obtained in the field by personnel of the Geological Survey, information on many wells and borings has been supplied by municipal and state agencies. Also, Metcalf and Eddy, Whitman and Howard, Weston and Sampson, consulting engineering firms in Boston, kindly furnished additional data on wells and borings. Further, R.E. Chapman Co., Oakdale, Mass.; Layne-New England Co., Arlington, Mass.; and D.L. Maher Co., North Reading, Mass., drilling companies, generously supplied logs and other records of wells.

The authors thank all those who supplied data and those who allowed personnel of the Geological Survey to install equipment and to collect data on their property.

\section{DEFINITION OF TERMS}

Definition of terms related to streamflow, water quality, and other hydrologic data, as used in this report, are defined as follows:

Color is expressed in units of the platinum-cobalt scale proposed by Hazen (1892, p. 427-428). A unit of color is produced by 1 milligram per liter of platinum in the form of the chloroplatinate ion.

The extent to which water is colored by material in solution is reported as part of the water analysis because a significant color in water may indicate the presence of organic material that may have some bearing on the dissolved-solids content.

Cubic foot per second (cfs) is the rate of discharge representing a volume of 1 cubic foot passing a given point during $I$ second and is equivalent to 7.48 gallons per second, 448.8 gallons per minute, or 646,317 gallons per day.

Discharge is the volume of water that passes a given point at a particular instant of time.

Drainage area of a stream at a specified location is that area, measured in a horizontal plane, enclosed by a topographic divide from which direct surface runoff from precipitation normally drains by gravity into the stream above the specified point. Figures of drainage area given herein include all closed basins, or noncontributing areas, within the area unless otherwise noted.

Gaging station is a particular site on a stream where systematic observations of gage height or discharge are obtained. When used in connection with a discharge record, the term is applied only to those gaging stations where a continuous record of discharge is obtained.

Hardness of water is a physical-chemical characteristic attributable to the presence of alkaline earths (principally calcium and magnesium) and is expressed as equivalent calcium carbonate $\left(\mathrm{CaCO}_{3}\right)$. 
Hardpan is a term commonly applied by New England drillers to a glacial deposit that resists penetration by light drilling equipment. The material is commonly till.

Micrograms per liter $(\mu g / I, U G / L)$ is a precise unit for expressing the concentration of chemical constituents in solution. One thousand micrograms per liter is equivalent to 1 milligram per liter. See below.

Milligrams per liter $(\mathrm{mg} / \mathrm{I}, \mathrm{MG} / \mathrm{L})$ is a unit for expressing the concentration of chemical constituents in solution. Milligrams per liter represents the weight of solute per unit volume of water. Milligrams or micrograms per liter may be converted to milliequivalents (one thousandth of a gram-equivalent weight of a constituent) per liter by multiplying by the factors in the table below. Concentration of suspended sediment expressed in milligrams per liter is based on the weight of sediment in a liter of water-sediment mixture.

\begin{tabular}{|c|c|c|c|}
\hline \multicolumn{3}{|c|}{ Multiply } & Multiply \\
\hline Ion & by & Ion & by \\
\hline$\overline{\mathrm{Al} \text { uminum }(\mathrm{Al}}{ }^{\mathrm{T}}$ & 0.11119 & Lead $\left(\mathrm{Pb}^{+2}\right)^{*}$ & 0.0096 \\
\hline Bicarbonate $\left(\mathrm{HCO}_{3}^{-1}\right) \ldots$ & .01639 & Lithium $\left(\mathrm{Li}^{+1}\right) *$. & .1441 \\
\hline Calcium $\left(\mathrm{Ca}^{+2}\right) \ldots$ & .04990 & Magnesium $\left(\mathrm{Mg}^{+2}\right)$ & .082 \\
\hline Carbonate $\left(\mathrm{CO}_{3}^{-2}\right)$ & .03333 & Manganese $\left(\mathrm{Mn}^{+2}\right) *$. & .036 \\
\hline Chloride $\left(\mathrm{Cl}^{-1}\right) \ldots$ & .02821 & Nitrate $\left(\mathrm{NO}_{3}^{-1}\right) \ldots$ & .016 \\
\hline Chromium $\left(\mathrm{Cr}^{+6}\right)^{*} \ldots \ldots \ldots$ & .11539 & Potassium $\left(\mathrm{K}^{+1}\right) \ldots$ & .025 \\
\hline Cobalt $\left(\mathrm{Co}^{+2}\right) * \ldots$ & .03394 & Sodium $\left(\mathrm{Na}^{+1}\right) \ldots .$. & .0435 \\
\hline Copper $\left(\mathrm{Cu}^{+2}\right) * \ldots$ & .03148 & Strontium $\left(\mathrm{Sr}^{+2}\right) * \ldots .$. & .0228 \\
\hline Fluoride $\left(\mathrm{F}^{-1}\right)$ & .05264 & Sulfate $\left(\mathrm{SO}_{4}^{-2}\right) \ldots$ & .0208 \\
\hline $\operatorname{Iron}\left(\mathrm{Fe}^{+3}\right)^{*} \ldots \ldots \ldots \ldots \ldots$ & .05372 & Zinc $\left(\mathrm{Zn}^{+2}\right) * \ldots \ldots \ldots$ & .03060 \\
\hline
\end{tabular}

*Constituent reported in micrograms per liter; multiply by factor and divide results by 1,000 .

$\mathrm{pH}$ is a symbol denoting the relative concentration of hydrogen ions in a solution; $\mathrm{pH}$ values range from 0 to 14 --the lower the value, the more acid is the solution; i.e., the more hydrogen ions it contains.

Refusal is a drilling term indicating the depth of a drill hole at which further penetration is impossible or impractical with the equipment being used.

Specific conductance is a measure of the ability of a water to conduct an electrical current and is expressed in micromhos per centimeter at $25^{\circ} \mathrm{C}$. Because the specific conductance is related to the number and specific chemical types of ions in solution, it can be used for approximating the dissolved-solids content in the water. Commonly, the amount of dissolved solids (in milligrams per liter) is about 65 percent of the specific conductance (in micromhos). This relation is not constant from stream to stream or from well to well, and it may even vary at a sampling site with changes in the composition of the water. 
Temperature. To convert temperature data shown in degrees Celsius (centigrade, ${ }^{\circ} \mathrm{C}$ ) to degrees Fahrenheit $\left({ }^{\circ} \mathrm{F}\right)$, see following table:

\begin{tabular}{|c|c|c|c|c|c|c|c|}
\hline${ }^{\circ} \mathrm{C}$ & $o_{F}$ & ${ }^{\circ} \mathrm{C}$ & ${ }^{\circ} F$ & ${ }^{\circ} \mathrm{C}$ & ${ }^{\circ} \mathrm{F}$ & ${ }^{\circ} \mathrm{C}$ & ${ }^{\circ} \mathrm{F}$ \\
\hline 0.0 & 32 & 10.0 & 50 & 20.0 & 68 & 30.0 & 86 \\
\hline .5 & 33 & 10.5 & 51 & 20.5 & 69 & 30.5 & 87 \\
\hline 1.0 & 34 & 11.0 & 52 & 21.0 & 70 & 31.0 & 88 \\
\hline 1.5 & 35 & 11.5 & 53 & 21.5 & 71 & 31.5 & 89 \\
\hline 2.0 & 36 & 12.0 & 54 & 22.0 & 72 & 32.0 & 90 \\
\hline 3.0 & 37 & 13.0 & 55 & 23.0 & 72 & 33.0 & 91 \\
\hline 3.5 & 38 & 13.5 & 56 & 23.5 & 74 & 33.5 & 92 \\
\hline 4.0 & 39 & 14.0 & 57 & 24.0 & 75 & 34.0 & 93 \\
\hline 4.5 & 40 & 14.5 & 58 & 24.5 & 76 & 34.5 & 94 \\
\hline 5.0 & 41 & 15.0 & 59 & 25.0 & 77 & 35.0 & 95 \\
\hline 5.5 & 42 & 15.5 & 60 & 25.5 & 78 & 35.5 & 96 \\
\hline 6.0 & 43 & 16.0 & 61 & 26.0 & 79 & 36.0 & 97 \\
\hline 6.5 & 44 & 16.5 & 62 & 26.5 & 80 & 36.5 & 98 \\
\hline 7.0 & 45 & 17.0 & 63 & 27.0 & 81 & 37.0 & 99 \\
\hline 8.0 & 46 & 18.0 & 64 & 28.0 & 82 & 28.0 & 100 \\
\hline 8.5 & 47 & 18.5 & 65 & 28.5 & 83 & 38.5 & 101 \\
\hline 9.0 & 48 & 19.0 & 66 & 29.0 & 84 & 39.0 & 102 \\
\hline 9.5 & 49 & 19.5 & 67 & 29.5 & 85 & 39.5 & 103 \\
\hline
\end{tabular}

Till is a geologic term for a glacial deposit of predominantly unsorted, unstratified material ranging in size from boulders to clay, commonly so compact that it is difficult to penetrate with light drilling equipment.

\section{SELECTED EQUIVALENTS}

1 cubic foot per second $=7.48$ gallons per second 449 gallons per minute 0.646 million gallons per day 86,400 cubic feet per day

1 million gallons per day $=1.55$ cubic feet per second 92.8 cubic feet per minute 0.134 million cubic feet per day 694 gallons per minute

1 milligram per liter $=1$ part per million 
Brown, Eugene, Skougstad, M.W., and Fishman, M.J., 1970, Methods for collection and analysis of water samples for dissolved minerals and gases: U.S. Geol. Survey Techniques of Water-Resources Inv., book 5, chap. Al, $160 \mathrm{p}$.

Carter, R.W., and Davidian, Jacob, 1968, General procedure for gaging streams: U.S. Geol. Survey Techniques of Water-Resources Inv., book 3, chap. A6, $13 \mathrm{p}$.

Colby, B.R., 1963, Fluvial sediments--a summary of source, transportation deposition, and measurement of sediment discharge: U.S. Geol. Survey Bull. 1181-A, $47 \mathrm{p}$.

Corbett, D.M., and others, 1943, reprinted 1957, Stream-gaging procedure a manual describing methods and practices of the Geological Survey: U.S. Geol. Survey Water-Supply Paper 888, $245 \mathrm{p}$.

Hazen, Allen, 1892, A new color standard for natural waters: Am. Chem. Jour., v. 12, p. $427-428$.

Hem, J.D., 1970, Study and interpretation of the chemical characteristics of natural water, 2d ed.: U.S. Geol. Survey Water-Supply Paper 1473, 363 p.

Langbein, W.B., and Iseri, K.T., 1960, General introduction and hydrologic definitions: U.S. Geol. Survey Water-Supply Paper 154l-A, 29 p.

Rainwater, F.H., and Thatcher, L.L., 1960, Methods for collection and analysis of water samples: U.S. Geol. Survey Water-Supply Paper 1454 , $301 \mathrm{p}$.

U.S. Geological Survey, 1954, Compilation of records of surface waters of the United States through September 1950, pt. 1-A, North Atlantic slope basins, Maine to Connecticut: U.S. Geol. Survey Water-Supply Paper 1301, $380 \mathrm{p}$.

1964, Compilation of records of surface waters of the United States October 1950 to September 1960, pt. 1-A, North Atlantic slope basins, Maine to Connecticut: U.S. Geol. Survey Water-Supply Paper 1721, 317 p.

1966-73, Water resources data for Massachusetts, New Hampshire, $\overline{\text { Rhode }}$ Island, Vermont, 1965-71: Boston, Mass., Water Resources Div., issued annually.

1969, Surface water supply of the United States 1961-65, pt. I, v. I, North Atlantic slope basins, Maine to Connecticut: U.S. Geol. Survey Water-Supply Paper 1901, $1027 \mathrm{p}$. 


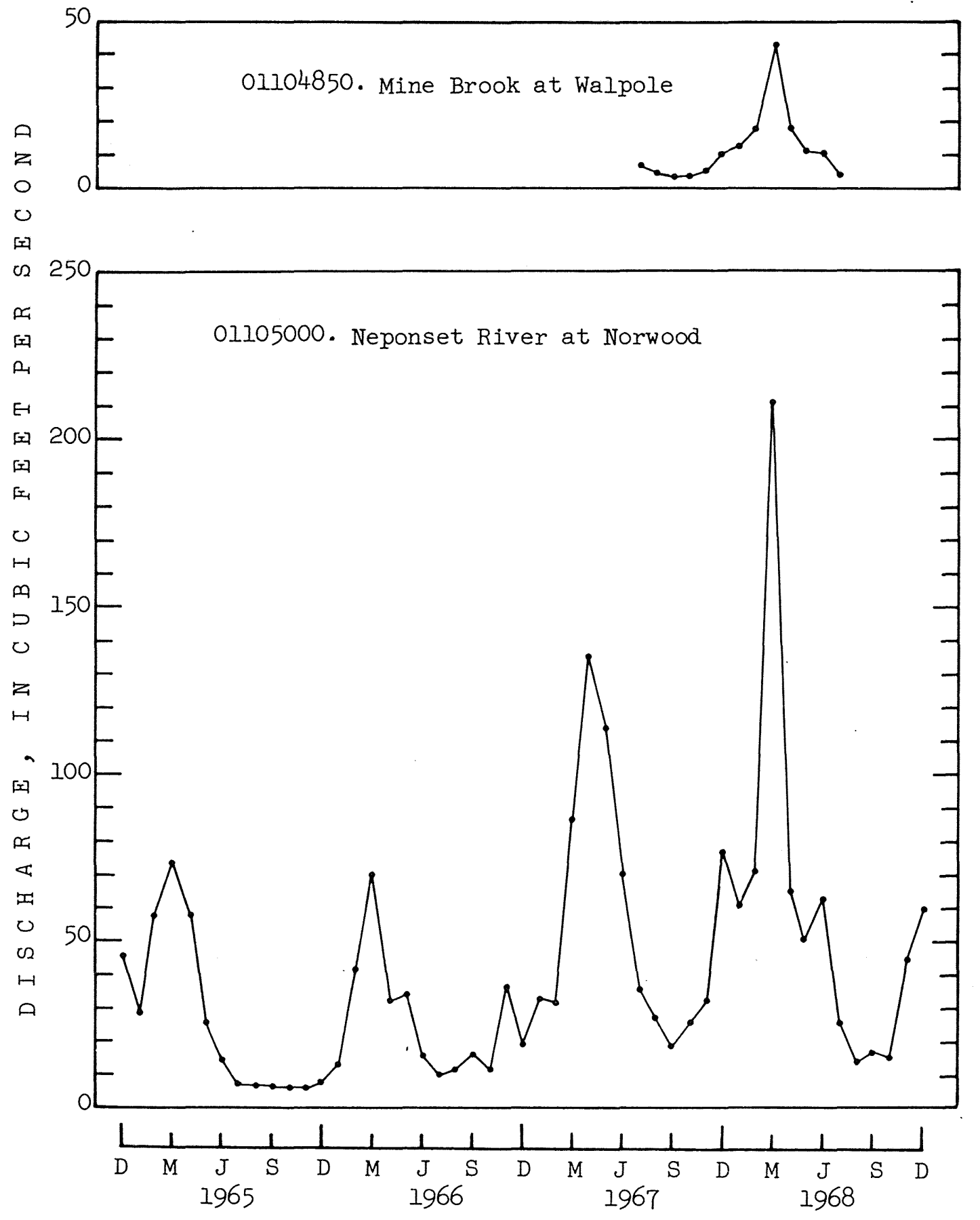

Figure 1.--Monthly mean discharge at stream-gaging stations and monthend water level in selected wells in the Neponset and Weymouth River basins, 1965-68 


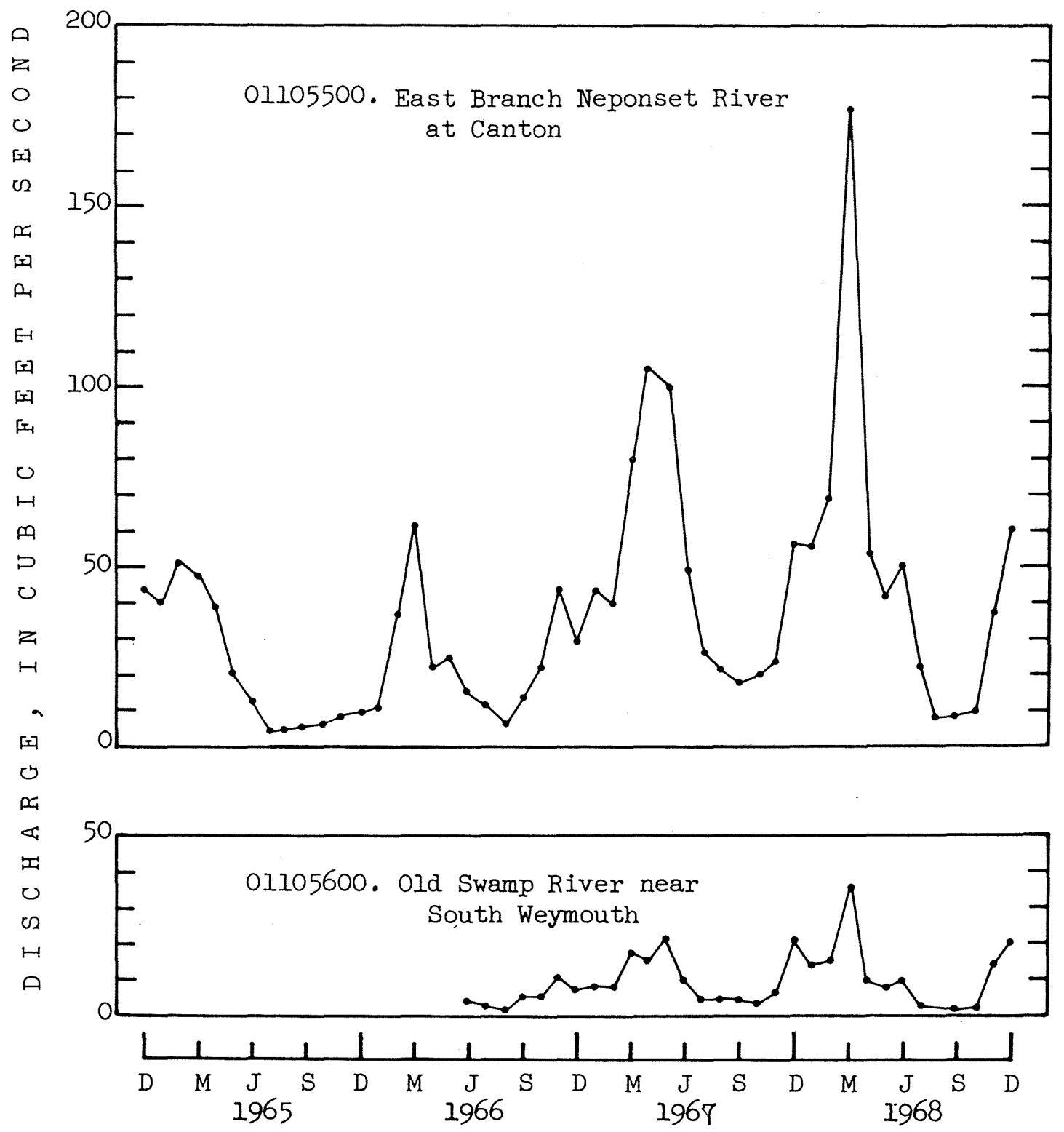

Figure 1.--Monthly mean discharge at stream-gaging stations and monthend water level in selected wells in the Neponset and Weymouth River basins, 1965-68.--Continued 


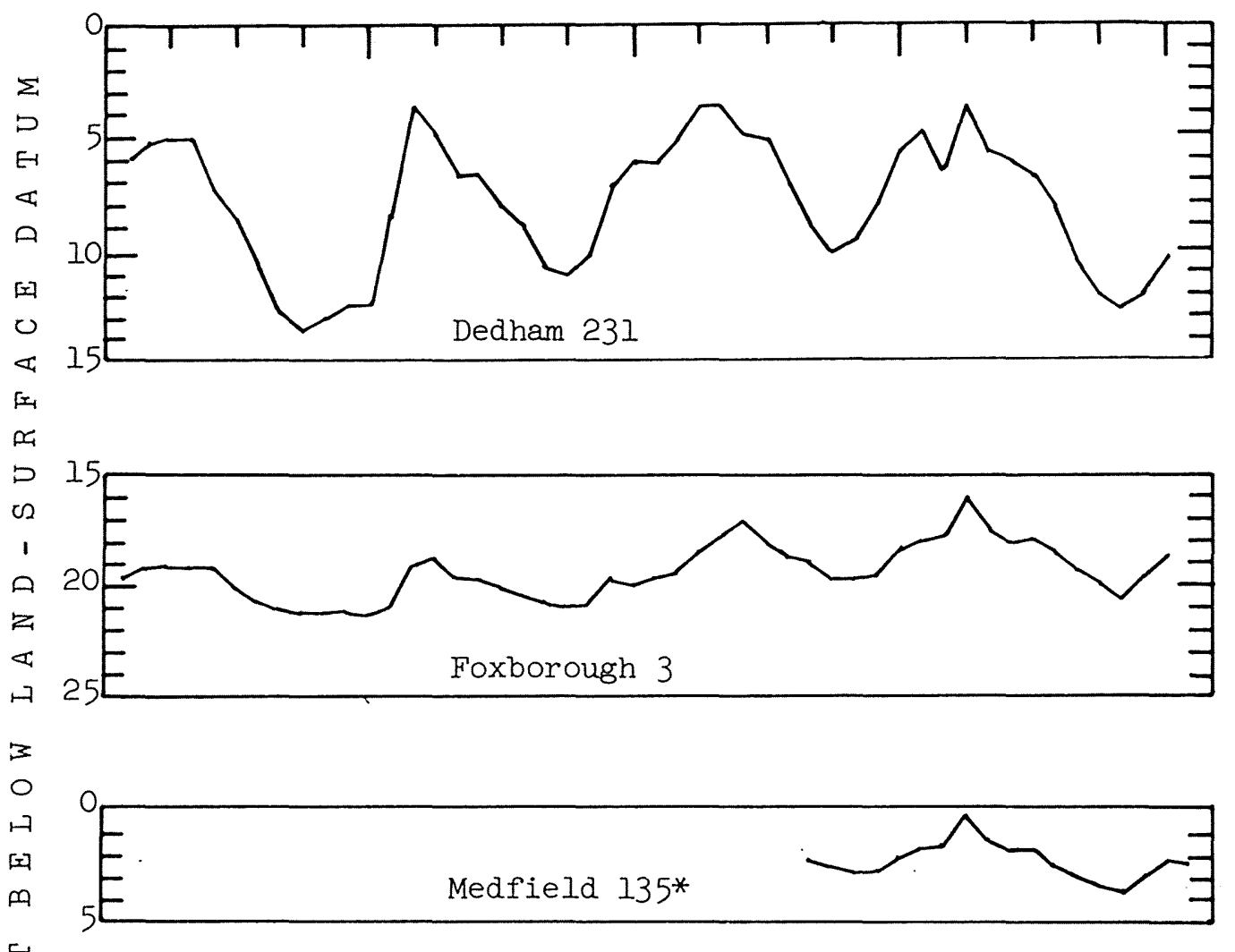

[a]

国

[r.

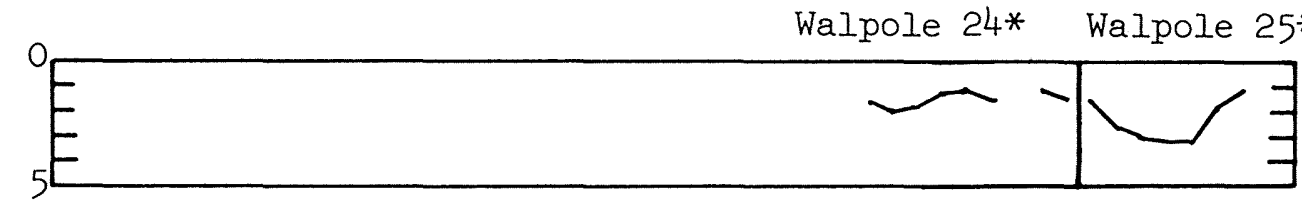

$\rightarrow$

보

$>$

国

$\mapsto$

$\alpha_{4}$

[x]

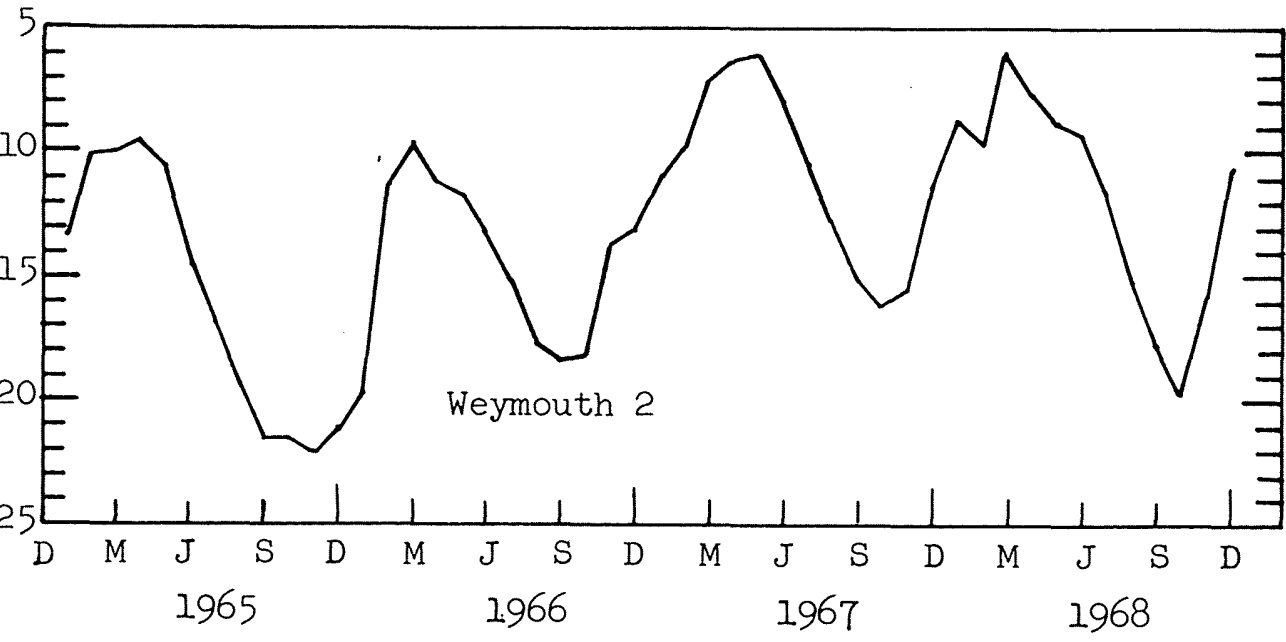

* Well equipped with continuous water-stage recorder.

+ Water level adjusted for difference between land-surface elevations at Walpole 24 and Walpole 25. Water level affected, at times, by ground-water pumpage.

Figure 1.--Monthly mean discharge at stream-gaging stations and monthend water level in selected wells in the Neponset and Weymouth River basins, 1965-68.--Continued 


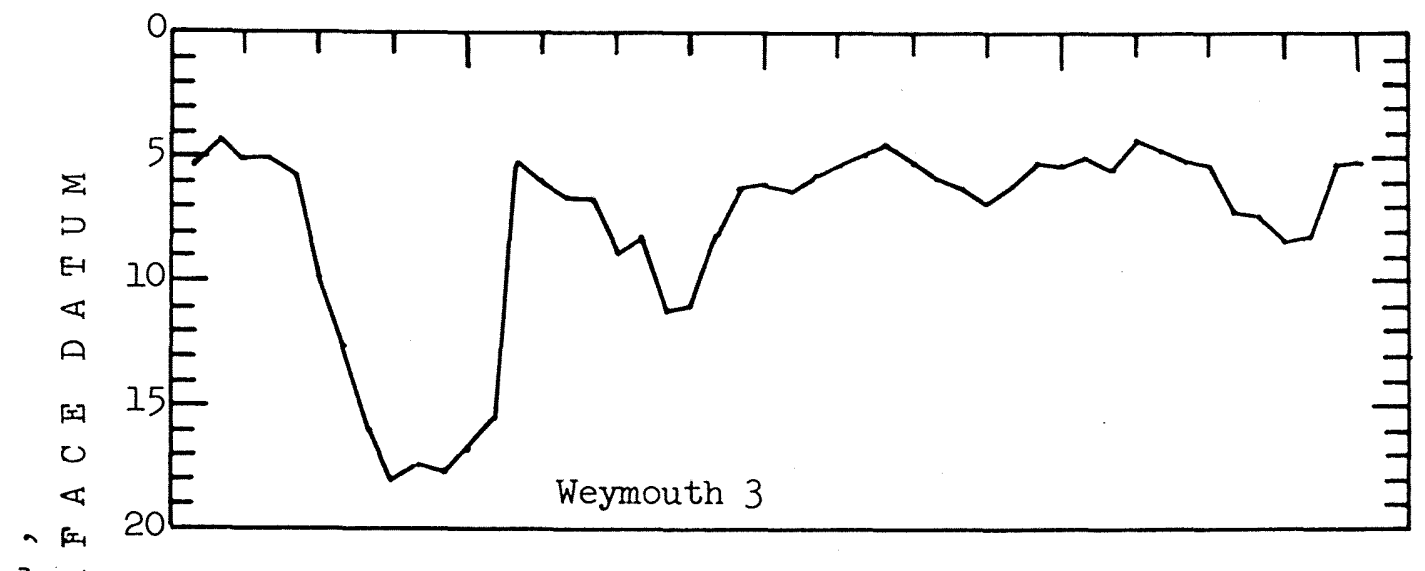

다 $p$

$P \quad 0$

되 1

$\mapsto \cap$

质

田

되 17

43

30
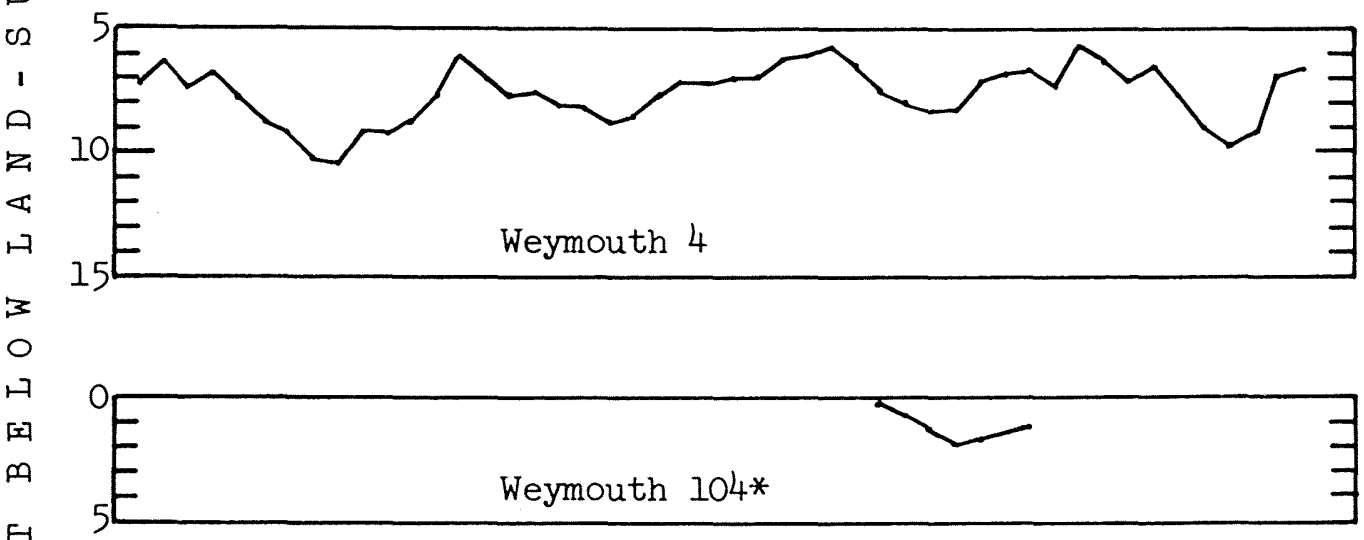

도

[ㄷ

Fr

年

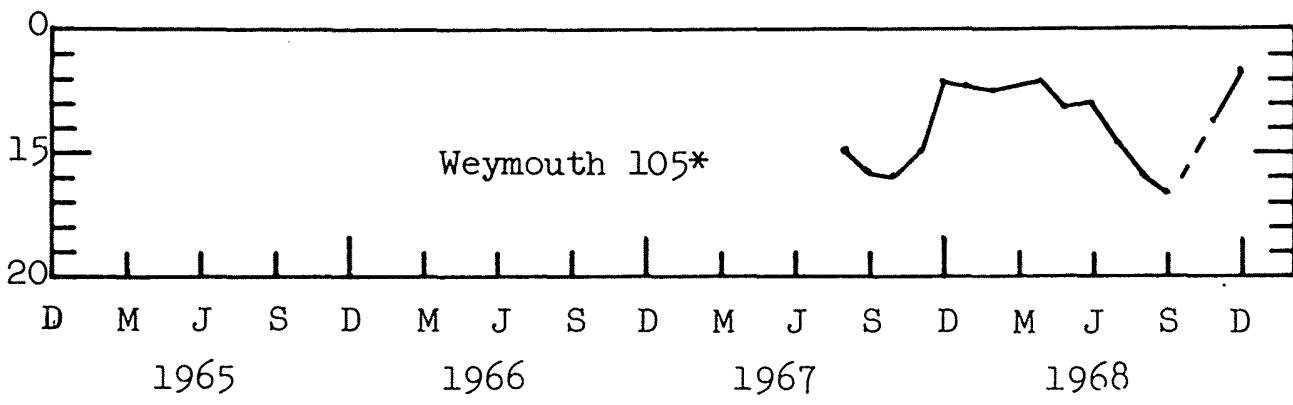

* Well equipped with continuous water-stage recorder.

Figure 1.--Monthly mean discharge at stream-gaging stations and monthend water level in selected wells in the Neponset and Weymouth River basins, 1965-68.--Continued 
LOCAL WELL NUMBER: LETTER PREFIX INDICATES--A, U.S. GEOLOGICAL SURVEY AUGER BORING; B, BRIDGE BORING; R, ROADWAY BORING; $W$, WELL OR TEST WELL (THE "W" IS OMITTED FROM PLATE I TO CONSERVE SPACE); $X$, MISCELLANEOUS TEST BORING.

LATITUDE-LONGITUDE: NUMBER FOLLOHING DECIMAL POINT INDICATES NUMBER OF WELLS OR BORINGS IN A 1 -SECOND GRID.

ALTITUDE OF LAND-SURFACE DATUM: ALTITUDES ARE EXPRESSED IN FEET ABOVE HEAN SEA LEVEL; THOSE PRECEDED BY a MINUS SIGN ARE BELOW MEAN SEA LEVEL.

METHOD DRILLED: A, AIR-ROTARY; B, BORED OR AUGERED; C, CABLE TOOL; D, DUG; H, HYDRAULIC-ROTARY; J, JETTED; P, AIRPERCUSSION; R, REVERSE-ROTARY; T, TRENCHED; $V$, DRIVEN; W, DRIVE-WASH.

WELL FINISH: C, POROUS CONCRETE; F, GRAVEL WALL WITH PERFORATED OR SLOTTED CASING; G, GRAVEL WALL WITH COMMERCIAL SCREEN H, HORIZONTAL GALLERY OR COLLECTOR; 0 , OPEN END; P, PERFORATED OR SLOTTED CASING; S, SCREEN; T, SAND POINT; W, WALLED OR SHORED; $X$, OPEN HOLE IN AQUIFER (GENERALLY CASED TO AQUIFER).

WELL DEPTH: DEPTH OF FINISHED WELL, IN FEET BELOW LAND SURFACE.

WELL USE: A, ANODE; D, DRAINAGE; G, SEISMIC HOLE; H, HEAT RESERVOIR; O, OBSERVATION; P, OIL OR GAS; R, RECHARGE; T, TEST; $U$, UNUSED; $W$, WATER WITHDRAWAL; $X$, WASTE DISPOSAL; $Z$, DESTROYED.

WATER-BEARING MATERIAL: PRINCIPAL WATER-BEARING ZONE.
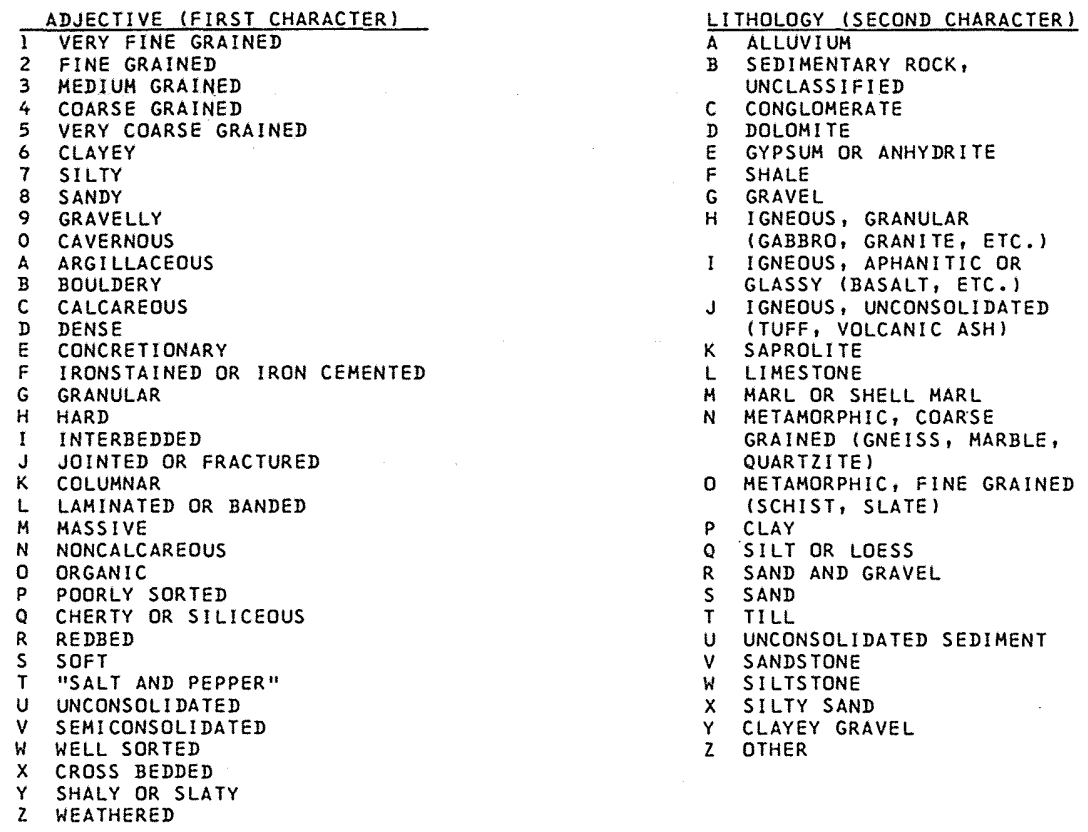

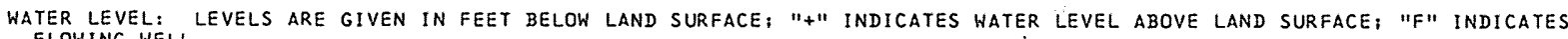
FLOWING WELL.

WATER USE: A, AIR CONDITIONING; B, BOTTLING; C, COMMERCIAL; D, DEWATERING; E, POWER GENERATION; F, FIRE PROTECTION; $H$, DOMESTIC; 1 , IRRIGATION; M, MEDICINAL; $N$, INDUSTRIAL (INCLUDES MINING); P, PUBLIC SUPPLY; R, RECREATION; S, STOCK; $T$, INSTITUTIONAL; $U$, UNUSED; $V$, REPRESSURIZATION; $W$, RECHARGE; $X$, DESALINATION--PUBLIC SUPPLIES; Y, DESALINATION--OTHER SUPPLIES.

PUMPAGE/YIELD: IN GALLONS PER MINUTE (GPM).

PUMPAGE/DRAWDOWN: THE DIFFERENCE BETWEEN STATIC WATER LEVEL AND PUMPING LEVEL.

PUMPAGE/TIME: THE FOLLOWING CODES ARE USED FOR PUMPING PERIODS OF LESS THAN 1 HOUR: A, THROUGH 15 MINUTES; B, 16 TO 30 MINUTES; $C, 31$ TO 45 MINUTES; $D, 46$ TO 59 MINUTES.

LOG: D, DRILLER'S LOG; E, ELECTRIC LOG; G, GEOLOGIST'S LOG AVAILABLE IN TABLE 2.

OW: TYPE OF CHEMICAL ANALYSIS AVAILABLE IN TABLE 3. C, COMPLETE; J, CONDUCTANCE AND CHLORIDE; K, CONDUCTANCE;

L, CHLORIDE; M, MULTIPLE (INCLUDES ONE COMPLETE AND ONE OR MORE PARTIAL); P, PARTIAL. 


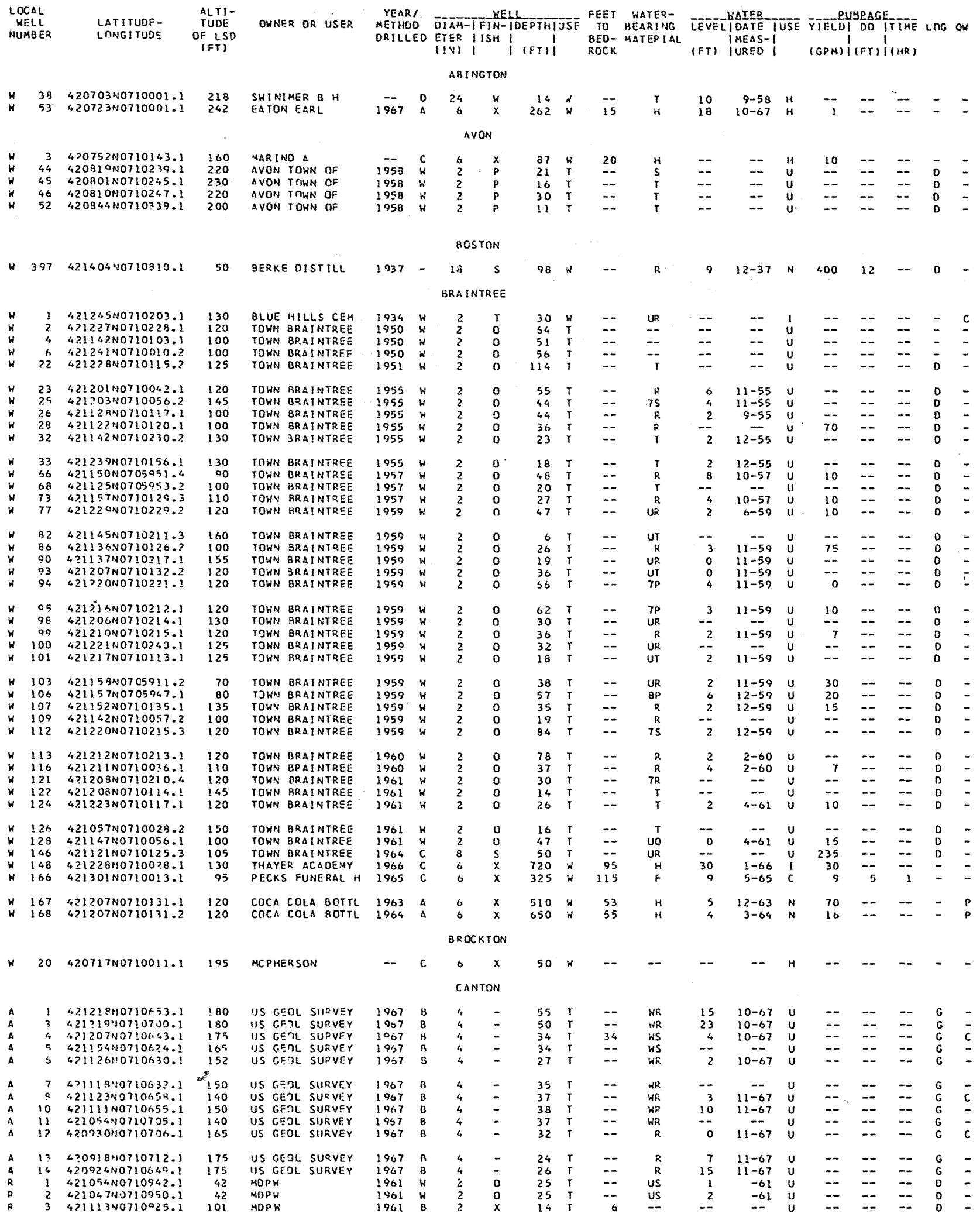




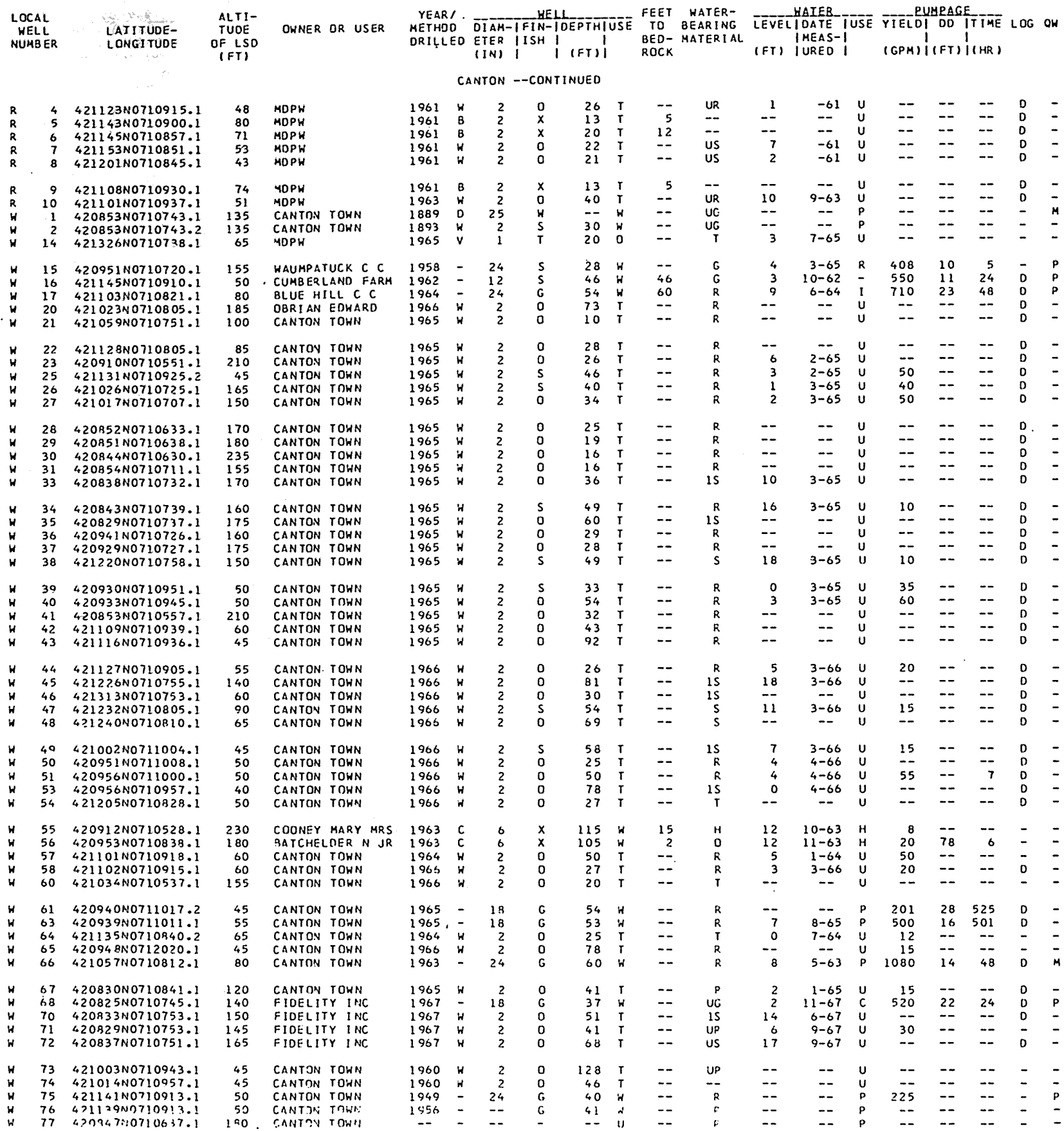

\begin{tabular}{|c|c|c|c|c|c|c|c|c|c|c|c|c|c|c|c|c|c|c|c|}
\hline$w$ & 17 & $\angle 213401071195 ! \ldots$ & 100 & SDLTZ AF THUS $C$ & 1040 & c & 90 & $x$ & 30 & 2 & 18 & $H$ & 26 & $7-47$ & $H$ & 120 & 2 & 1 & D \\
\hline & 14 & 42133840711042.1 & 125 & BRUCE ROONALO OR & 1040 & c & 6 & $x$ & 235 & * & -- & $H$ & 40 & $7-47$ & H & 15 & -- & -- & - \\
\hline$W^{\prime}$ & 15 & $421351 N 0711100.1$ & 120 & DUINLAN FOANC IS & lo3s & $\mathrm{v}$ & 1 & $n$ & 30 & 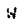 & -- & 2 & -- & $\ldots$ & c & - & -- & -- & 0 \\
\hline$W$ & $2 ? 1$ & $4 ? 1250$ NO712000.! & 65 & US GEOt SUFVEY & :9k4 & 3 & a. & $s$ & 22 & 2 & -- & 0 & 12 & $11-64$ & $u$ & -- & -- & -- & G \\
\hline & 2.32 & 421301110713920.1 & 49 & DEDHIY WATFO 5.$)$ & $10 \times 3$ & $n$ & 2 & 0 & hS & $i$ & -- & UP & -- & -- & $u$ & - & -- & -- & 0 \\
\hline & 263 & $421717 N 0710857$. & 45 & DEDHA' WATCR CO & 1963 & $w$ & 2 & 0 & 34 & $T$ & -- & up & - & -- & u & -- & -- & -- & 0 \\
\hline & 264 & $421420 \times(71022 ! .1$ & 145 & SULI IVAN J D & 1952 & c & 6 & $x$ & 170 & $\dot{n}$ & 14 & -- & 14 & $11-52$ & H & 10 & - & -- & - \\
\hline
\end{tabular}




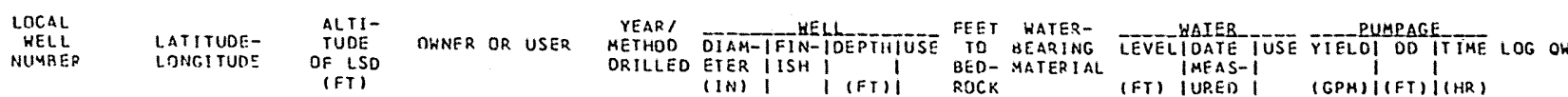
DOVER DOVER HATER CO -- $2 S$

$3 \quad 420432 N 0711512.12290$ $\begin{array}{lll}0 & 420442 N 0711418.1 & 275 \\ 0 & 430440 N 0711419.1 & 275\end{array}$ $\begin{array}{lll}11 & 470445 N 07114 ? ! 11 & 275 \\ 20 & 420534 \times 07114 ! 10.1 & 270\end{array}$ $21420531 N 0711410.1 \quad 270$ $\begin{array}{lll}22 & 420525 N 0711419.1 & 270 \\ 24 & 420531 N 0711511.1 & 270\end{array}$ $\begin{array}{lll}24 & 420531 N 0711511.1 & 270 \\ 40 & 420439 N 0711423.1 & 280 \\ 47 & 420445 N 0711421.2 & 270\end{array}$ al 4?0452N0711415.1 275 $67420425 N 0711506.1 \quad 385$ $\begin{array}{lll}68 & 420450 N 0711437.1 & 275 \\ 70 & 420425 N 0711526.1 & 286\end{array}$ $71420427 N 0711530.1 \quad 290$ $\begin{array}{lll}73 & 420479 \times 07115 ? 8.1 & 290 \\ 75 & 420437 \times 1071153 ? .1 & 300\end{array}$

2
16
16
16
2

$\begin{array}{rrrr}\text { B } & \text { I } & 21351407054 \geqslant 1.1 & 35 \\ \text { W } & 7 ? 095040705507.1 & 135\end{array}$ $\begin{array}{llll}W & 80 & 421315 N 0705410.1 & 81 \\ H & 81 & 421231 N 070543.1 & 42\end{array}$ $H \quad 105 \quad 421343407654 ! 7.120$

H $105 \quad 4 ? 13 ! 7 \times 10705426.1$ $H \quad 167$
$H \quad 169$
$H$ $H \quad 169$
$H \quad 1821240+13705454.1$
$H$

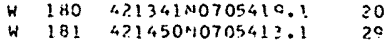
H $187 \quad 47004940705447.1 \quad 168$

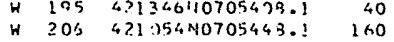

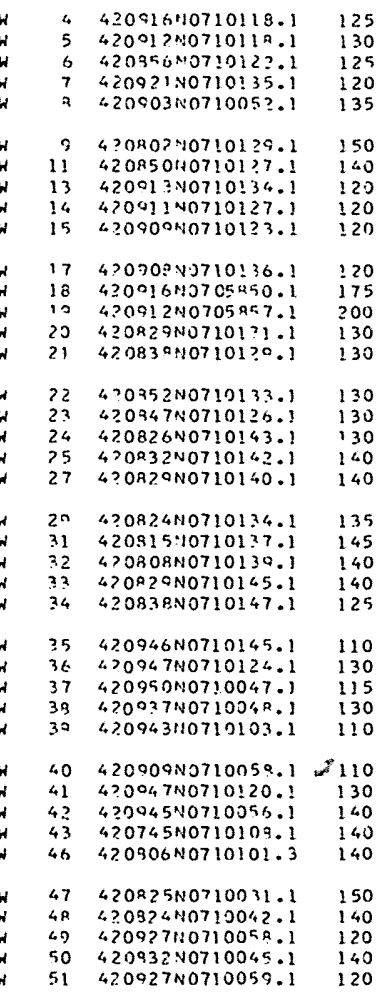
HINTHA RAT CO $\begin{array}{ll}1935 & v \\ 1954 & 0 \\ 1954 & w \\ 1957 & w \\ 1957 & w \\ 1966 & -1 \\ 1966 & w \\ 1957 & 4 \\ 1957 & w \\ 1964 & = \\ 1965 & - \\ 1960 & c\end{array}$

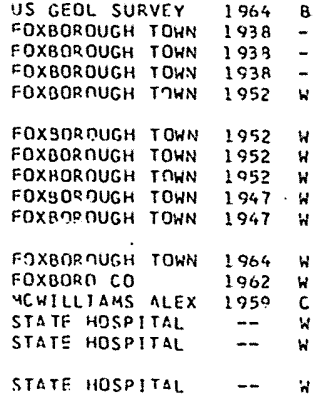$$
\begin{array}{ll}
2 & 0 \\
2 & 0 \\
2 & n \\
2 & n
\end{array}
$$$$
2
$$

STATF HOSPITAL

STATE HOSPITAL -..

${ }_{40 \mathrm{NW}}$

$$
\begin{array}{rrrr}
32 & U & -- & \text { IS } \\
46 & H & -- & \text { UR } \\
40 & H & -- & \text { UR } \\
42 & W & -- & \text { UR } \\
35 & T & -- & \text { UR } \\
24 & Y & -- & \text { UR } \\
26 & T & -- & \text { UR } \\
25 & T & -- & R \\
31 & T & -- & \text { UR } \\
61 & T & -- & \text { UR } \\
43 & T & -- & R \\
32 & T & -- & R \\
130 & H & 26 & H \\
10 & T & -- & 6 S \\
7 & T & -- & 7 R \\
16 & T & -- & T
\end{array}
$$

HINGHAM HIHCHAY WAT COD MINSHAM WAT CO HINGHAM HAT CO
HINGHA' WAT CO HINGHA'S WAT CO HINGHAY WAT COO $\begin{array}{ll}\text { CANERON ROY B } & 1964 \\ \text { HOOPEY DONALD } & 1965 \\ \text { HICKEY EDHARD Y } & 1960\end{array}$

$\begin{array}{rl}1 & 0 \\ 30 & 0 \\ 2 & 0 \\ 2 & 0 \\ 2 & 0 \\ z & 0 \\ 2 & 0 \\ 2 & 0 \\ 2 & 0 \\ 2 & 0\end{array}$

$\begin{array}{llll}14 & T & -- & -- \\ 13 & U & -- & R \\ 32 & T & -- & -- \\ 26 & T & -- & 35\end{array}$

$\begin{array}{llll}20 & T & -- & 3 S \\ 20 & T & -- & 9 S\end{array}$

$\begin{array}{llll}36 & T & -- & 3 r \\ 46 & T & -- & 2 R\end{array}$

$\begin{array}{llll}48 & T & - & 2 R \\ 20 & T & -- & 28\end{array}$

\begin{tabular}{|c|c|c|c|c|c|c|c|c|c|c|c|c|c|c|c|c|c|}
\hline IALLGROOK & TCWN & 1954 & $w$ & 2 & 0 & 29 & T & -- & UT & 5 & $9-54$ & w & -- & -- & -- & D & - \\
\hline HOLRRDOK & TOHN & 1954 & w & ? & 0 & 27 & $T$ & - & UR & 8 & $9-54$ & $u$ & 25 & $-\cdots$ & -- & 0 & - \\
\hline HOLEO.NOK & TOHN & 1954 & $w$ & 2 & 0 & 27 & T & -- & UK & 2 & $10-54$ & $u$ & -- & -- & -- & 0 & - \\
\hline HXLBRDOK & In:HN & 1954 & $\star$ & 2 & i) & 12 & $T$ & -- & -- & -- & -- & $u$ & -- & -- & -- & 0 & - \\
\hline HOLRROกK & TOWN & 1954 & $w$ & 2 & 0 & 34 & r & -- & $\dot{O} R$ & -- & - & $u$ & -- & -- & - & D & - \\
\hline HoL.90 3nK & TCWN & 1957 & H & ? & 0 & 22 & $T$ & -- & -- & -- & -- & $u$ & 20 & -- & -- & - & - \\
\hline $4018000 \mathrm{~K}$ & TOWN & 1957 & - & -- & ;, & 33 & $w$ & -- & $\mathrm{R}$ & -- & -- & $p$ & 250 & -- & -- & - & - \\
\hline HDLBRกาK & TOWN & 1954 & 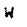 & 2 & 0 & 34 & $r$ & -- & $6 R$ & 3 & $10-54$ & $u$ & 20 & -- & -- & D & - \\
\hline HULBRDOK & TIN & 1954 & H & 2 & 0 & 69 & $r$ & -- & 65 & 4 & $10-54$ & u & -- & -- & -- & 0 & - \\
\hline HกLBRกOK & TOWN & 1954 & $w$ & 2 & 0 & 53 & $\mathrm{~T}$ & -- & of & 0 & $10-54$ & u & 5 & -- & - & $D$ & - \\
\hline $4 \cap L B=\pi \cap K$ & TCWN & 1954 & $w$ & 2 & 0 & 31 & $T$ & -- & UP & 3 & $10-54$ & $u$ & -- & -- & -- & D & - \\
\hline HOLBRTOK & TOWN & 1954 & $\because$ & 2 & 0 & 43 & $\mathrm{i}$ & -- & UP & 1 & $10-54$ & u & -- & -- & -- & D & - \\
\hline HOLGROOK & TONN & 1954 & H & 2 & $n$ & 21 & $T$ & -- & ur & -- & -- & u & -- & -- & -- & 0 & - \\
\hline HOLLBRכOK & THWN & 1964 & $h^{\prime \prime}$ & 2 & 0 & 39 & $T$ & -- & US & 1 & $4-64$ & u & -- & -- & -- & D & - \\
\hline HOLHROOK & TUNN & 1064 & $w$ & 2 & 0 & 27 & $T$ & -- & us & 2 & $4-64$ & u & -- & -- & -- & D & - \\
\hline HกLBRกOK & $T C W V$ & 1958 & - & 24 & G & $6 !$ & $w$ & -- & h & -- & -- & $\mathbf{p}$ & 700 & -- & -- & - & $p$ \\
\hline HOLARADK & rinn & 1.957 & - & -- & 6 & 53 & $w$ & -- & R & -- & -- & $p$ & 300 & -- & -- & - & - \\
\hline HOL IROOK & I CWN & 1964 & in & 2 & 0 & 19 & $T$ & - & is & -- & -- & u & - & -- & -- & 0 & - \\
\hline HOLBROOK & TOWN & 1364 & 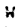 & 2 & 1) & 37 & $T$ & -- & UR & 9 & $4-64$ & $u$ & -- & -- & -- & 0 & - \\
\hline HกLBRกOK & TOWN & $196 t_{t}$ & i & 2 & 0 & 44 & $T$ & -- & s & -- & -- & u & -- & -- & -- & D & - \\
\hline HOLHEOOK & T NWN & 1964 & H & 2 & 0 & 29 & $\mathrm{~T}$ & -- & is & -- & -- & $u$ & -- & -- & -- & 0 & - \\
\hline HOLEROOK & TOWN & 1964 & H & 2 & c & 33 & $\mathrm{r}$ & -- & is & -- & - & $u$ & -- & -- & -- & 0 & - \\
\hline HOLBROOK & TOWN & 1064 & $\mathrm{H}$ & 2 & 0 & 51 & T & -- & us & 3 & $5-64$ & $u$ & -- & -- & -- & D & - \\
\hline 'HOLAFOOK & TOHN & 1964 & H & 2 & 0 & 50 & $T$ & - & us & 2 & $5-64$ & u & -- & -- & -- & 0 & - \\
\hline HOLARDOK & TCHN & 1964 & $w$ & 2 & 0 & 25 & $T$ & -- & is & 0 & $5-64$ & u & $\cdots$ & $\cdots$ & -- & 0 & - \\
\hline HOLBROOK & TOKN & 1964 & $\omega$ & 2 & 0 & 36 & $\mathrm{r}$ & - & 15 & 0 & $5-64$ & $u$ & -- & -- & -- & D & - \\
\hline HOLBRDOK & TOWN & 1964 & $w$ & 2 & 0 & 65 & T & -- & os & 9 & $5-64$ & $u$ & -- & -- & -- & D & - \\
\hline HOLBRDOK & TOWN & 1964 & W & 2 & 0 & 18 & $\mathrm{~T}$ & -- & UT & -- & -- & $u$ & -- & -- & -- & 0 & - \\
\hline HOLARNOK & TOWN & 1964 & 4 & 2 & 0 & 24 & $\mathrm{~T}$ & -- & UT & -- & - & u & -- & -- & -- & D & - \\
\hline HOLBRONK & TOWN & 1964 & in & 2 & 0 & 33 & $\mathrm{~T}$ & -- & UR & 3 & $5-64$ & $u$ & 25 & -- & 2 & D & - \\
\hline HOLBRONK & Town & 1964 & $W$ & 2 & 0 & 40 & $\mathrm{~T}$ & -- & is & 8 & $5-64$ & $u$ & -- & -- & -- & D & - \\
\hline HOLBROOK & TOWN & -- & $w$ & 2 & 0 & 41 & $\mathrm{~T}$ & -- & is & 0 & $5-64$ & $\mathrm{u}$ & -- & -- & -- & 0 & - \\
\hline HOLBRONK & TOWN & 1964 & $w$ & 2 & 0 & 41 & $\mathrm{~T}$ & - & us & -- & - & u & -- & -- & -- & D & - \\
\hline HOLBRONK & TRNN & 1964 & $w$ & 2 & 0 & 32 & $T$ & -- & US & 5 & $6-64$ & $u$ & -- & -- & -- & D & - \\
\hline HOLBR ODK & TOWN & 1965 & H & 2 & 0 & 42 & in & -- & $R$ & 2 & $12-65$ & $P$ & 60 & -- & 8 & 0 & $p$ \\
\hline HOLRROIIK & TCWN & 1957 & $w$ & 2 & 0 & 41 & $\mathrm{r}$ & -- & UR & 5 & $1-57$ & $u$ & 10 & -- & -- & 0 & - \\
\hline HOLBPDOK & T OWN & 1957 & 4 & 2 & 0 & 20 & $T$ & - & UT & - & -- & $u$ & -- & -- & -- & D & 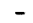 \\
\hline HOLAROOK & TOWN & 1957 & in & 2 & 0 & 26 & $\mathbf{T}$ & - & UP & -- & -- & $u$ & -- & -- & -- & 0 & - \\
\hline HOLBROOK & TOHN & 1957 & $*$ & 2 & 0 & 42 & $T$ & -- & UR & 5 & $1-57$ & u & 5 & -- & -- & 0 & - \\
\hline HOLRROOK & TOWN & 1957 & i & 2 & 0 & 27 & $\mathrm{~T}$ & -- & UP. & -- & -- & u & -- & -- & -- & $D$ & \\
\hline
\end{tabular}

$\begin{array}{rrrr}45 & T & - & R \\ 15 & -- & 3 S\end{array}$

$140 \mathrm{H} \quad 14 \quad \mathrm{H}$

$$
\begin{aligned}
& \begin{array}{rrrrrrrr}
21 & 11-64 & U & -- & -- & -- & G & - \\
0 & -38 & p & 400 & -- & -- & - & M \\
0 & -38 & P & 300 & -- & -- & - & p \\
0 & -38 & P & 300 & -- & -- & - & p \\
-- & -- & U & -- & -- & -- & D & -
\end{array} \\
& \begin{array}{llllllll}
-- & - & U & -- & -- & -- & 0 & - \\
- & -- & U & -- & -- & - & 0 & 0
\end{array}
\end{aligned}
$$

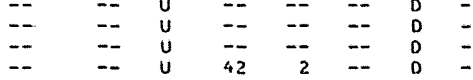

$$
\begin{aligned}
& \text { - } \quad \text { - } \\
& \begin{array}{ccccccc}
-- & u & 40 & - & 168 & - & - \\
8-62 & u & -- & -- & -- & 0 & -
\end{array} \\
& \begin{array}{ccccccc}
12-59 & H & 1 & =- & =- & 0 & - \\
-- & U & -- & =- & = & 0 & = \\
-- & u & -- & -- & -- & 0 & -
\end{array} \\
& =: \quad:=:=:
\end{aligned}
$$




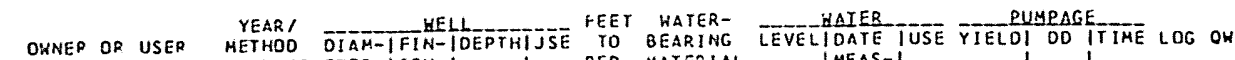

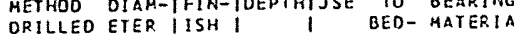

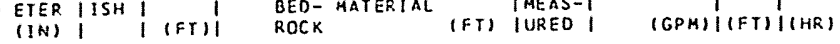

HOLBROOK --CONTINUED
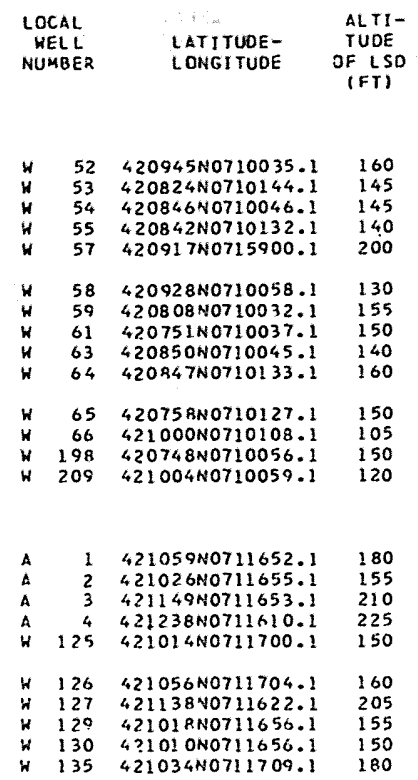

HOLBROOK TOKN HOLBROOK TCWN HOLBROOK TOHN HOLBROOK TOHN HOLBROOK TOHN HDLERODK TCWN HOLBROOK TOHN HOLBROOK TCHN HOLBROOK TOWN tocosoox roun HOLBROOK TOWN HOLBROOK TOHN $\begin{array}{lll}1957 & H & 2 \\ 1957 & H & 2 \\ 1958 & H & 2 \\ 1959 & H & 2 \\ 1959 & H & 2 \\ 1959 & H & 2 \\ 1959 & H & 2 \\ 1959 & H & 2 \\ 1959 & H & 2 \\ 1959 & H & 2 \\ 1961 & H & 2 \\ 1963 & K & 2 \\ 1959 & 4 & 2 \\ 1930 & H & 42\end{array}$ MEDF IELO

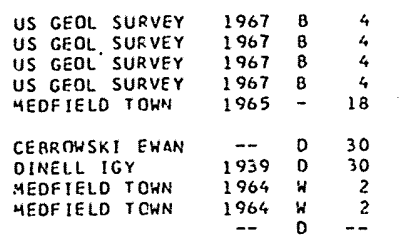

HORWOOD

US GEOL SURVEY US GEOL SURVEY US GEXL SUREEY US GEOL SURVEY
US GENL SUF VEY

US GFOL SURVEY US GEOL SURVEY US GEOL SURVEY NDRHOON TOKN

MORWODO TOWN NORWTOD TOWH NOPHOOD TOWN NORHOOO TOWN GELSO $P$ NORHOOD TOWN NORHOOD TOWN NORHOOD TOWN

NORHOON TOKM NORHIDDO TOHN NORWINOO TOWN NORHIOD TOWN NOR HOOD TOHN NORHODO TOWN NOP.KNOD TOWM NORHDOD TOWN
NORHODO TOHN NORHODO TDHN A AND P CO STAR MARKET
NIRHOOD TOWN

H $45421235 N 0711056$. 46
$4721208 N 0711098.1$
$421226 N 0711103.1$

$\begin{array}{lll}1967 & B & 4 \\ 1967 & B & 4 \\ 1966 & B & 4 \\ 1966 & B & 4 \\ 1966 & 8 & 4 \\ 1966 & 8 & 4 \\ 1966 & B & 4 \\ 1966 & A & 4 \\ 1939 & H & 2 \\ 1939 & H & 2 \\ 1939 & H & 2 \\ 1939 & H & 2 \\ 1939 & H & 2 \\ 1930 & H & 2 \\ 1939 & H & 2\end{array}$

$\begin{array}{rrr}1939 & 0 & 30 \\ 1939 & W & 2 \\ 1939 & W & 2 \\ 1937 & W & 2\end{array}$

1944

1944

1944

$1920 . \mathrm{H}$

1920
1920

$1925 \mathrm{~W}$

$1925 \mathrm{H}$

$1949-246$

$\begin{array}{rrrr}1949 & - & 24 & 6 \\ 1912 & - & -2 & 0 \\ 19 & - & 5\end{array}$

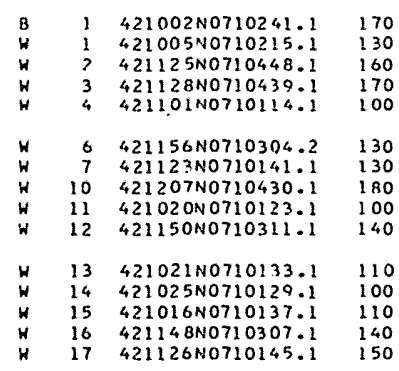

MOPW

RANODLPH TCEN RANOOLPH TOWN RANDOLPH YOHN
RANDOLPH TONN RANOOLPH TONN RANDOLPH TOWN RANODLPH TOHN RANDDLPH TOWN
P.ANONLPH TOWN RANDDLPH TOHN RANOOLPH TOHN RANDOLPH TOHN RANDOLPH TCWN
RANDOLPH TOHA

\begin{tabular}{|c|c|c|c|}
\hline 22 & $\mathrm{~T}$ & -- & ur \\
\hline 46 & $\mathrm{~T}$ & -- & 15 \\
\hline & r & -- & UR \\
\hline 24 & T & & UT \\
\hline 23 & $\boldsymbol{T}$ & - & R \\
\hline & I & 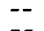 & ${ }^{R}$ \\
\hline 18 & T & -- & UT \\
\hline 37 & r & -. & UR \\
\hline 25 & $T$ & -- & -- \\
\hline 2 & Y & $\therefore$ & $\overline{7 s}$ \\
\hline & H & -- & -- \\
\hline
\end{tabular}

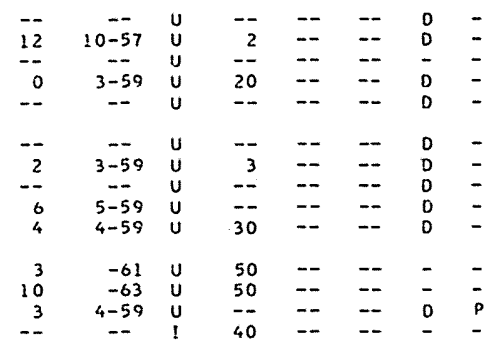

$\begin{array}{lllr}11 & T & -- & P R \\ 45 & Y & -- & 5 S \\ 44 & Y & -- & - \\ 58 & Y & -- & R \\ 60 & W & -- & U P\end{array}$

$\begin{array}{rrrrrrrr}5 & 11-67 & U & -- & -- & -- & G & C \\ 5 & 11-67 & U & -- & -- & -- & G & - \\ 12 & 11-67 & U & -- & -- & -- & G & - \\ 10 & 11-67 & U & -- & -- & -- & G & \bar{D} \\ 2 & 5-65 & P & 900 & 31 & 168 & D & D\end{array}$

$\begin{array}{cccc}44 & W & - & - \\ 14 & H & - & - \\ 40 & T & - & 3 R \\ 58 & T & - & 2 R\end{array}$

$\begin{array}{llll}40 & T & - & 3 R \\ 58 & T & - & 2 R \\ -- & 0 & -- & U R\end{array}$

$\begin{array}{ll}9-67 & 1 \\ 9-67 & 4 \\ 8-64 & \\ 9-64 & \\ 9-67 & \end{array}$

$\begin{array}{rrrrr}-- & -- & -- & - & c \\ -- & -- & -- & - & c \\ 60 & 1 & 3 & 0 & - \\ 60 & 2 & 3 & 0 & - \\ -- & -- & -- & - & -\end{array}$

$\begin{array}{llll}62 & T & -- & \text { WR } \\ 43 & T & -- & \text { WD } \\ 65 & T & -- & 35 \\ 336 & T & -- & \text { US } \\ 152 & T & -- & \text { US }\end{array}$

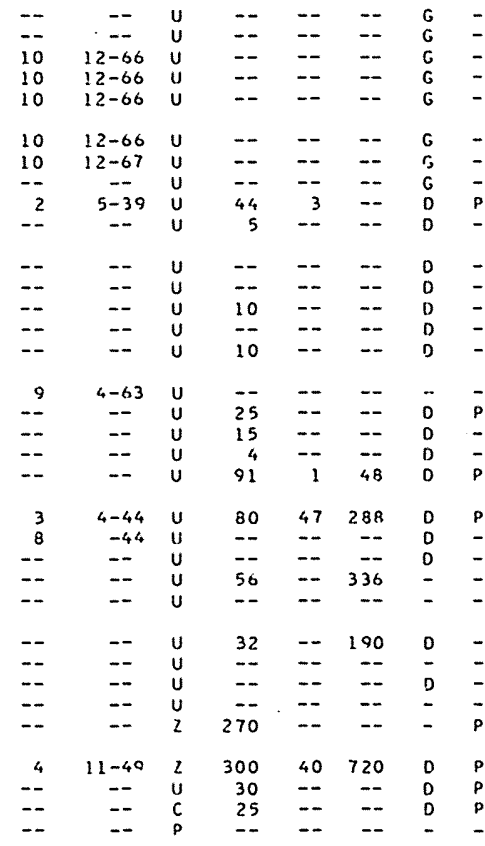

$\begin{array}{ll}87 & 1 \\ 47 & 1 \\ 83 & 1\end{array}$

$\begin{array}{ll}-- & \text { R } \\ - & \text { us } \\ -6 & R^{2}\end{array}$

$\begin{array}{ll}88 & \mathrm{~T} \\ 34 & \mathrm{~T}\end{array}$

88

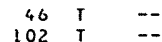

$\begin{array}{rrr}21 & T & 100 \\ 110 & T\end{array}$

$\begin{array}{lll}19 & 4 & --\end{array}$

$\begin{array}{lll}51 & \mathrm{~T} & = \\ 54 & \mathrm{I} & --\end{array}$

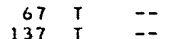

$\begin{array}{ccc}49 & \mathrm{~T} & =- \\ 34 & \mathrm{~T} & --\end{array}$

RANDOL PH

$\begin{array}{lll}40 & T & -- \\ 50 & T & --\end{array}$

$\begin{array}{llll}30 & \mathrm{~T} & -- & 25 \\ 50 & \mathrm{~T} & - & 15\end{array}$

$\begin{array}{llll}90 & \mathrm{H} & -- & -- \\ 50 & \mathrm{H} & -- & --\end{array}$

$\begin{array}{llll}62 & H & -- & -\end{array}$

$\begin{array}{llll}84 & 1 & = & 15 \\ 25 & W & = & \text { UR } \\ 35 & U & -- & \text { UR }\end{array}$

$\begin{array}{llll}1951 & W & 2 & 0 \\ 1957 & W & 2 & 0 \\ 1959 & H & 2 & 0 \\ 1959 & H & 2 & 0 \\ 1959 & H & 2 & 0 \\ 1956 & H & 2 & 0 \\ 1959 & H & 2 & 0 \\ 1959 & H & 2 & 0 \\ 1959 & H & 2 & 0 \\ 1956 & H & 2 & 0 \\ 1959 & H & 2 & 0 \\ 1959 & H & 2 & 0 \\ 1959 & H & 2 & 0 \\ 1956 & W & 2 & 0 \\ 1959 & H & 2 & 0\end{array}$

$\begin{array}{llll}13 & T & -- & T \\ 57 & T & -- & 76 \\ 24 & T & -- & \text { UT } \\ 21 & T & -- & \text { UT } \\ 23 & T & -- & \text { UT } \\ 43 & T & -- & \text { UR } \\ 43 & T & -- & \text { UR } \\ 145 & T & -- & \text { UT } \\ 45 & T & -- & 15 \\ 65 & T & 7 \\ 74 & T & - & 7 P \\ 67 & T & -- & 15 \\ 63 & T & -- & 15 \\ 60 & T & -- & 15 \\ 44 & T & -- & 15 \\ 25 & T & -- & 85\end{array}$

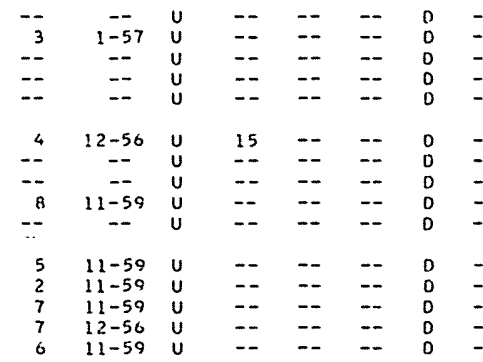


LOCAL
WFLL LATITUCE- TLTILONGITUDE

TIUDE OWNER OR USFP

YEAR'

NUMBER (FT) METHOO OIAM-IFIN-IDEA

DIAM-IFIN-IDEPTHIUSE TO DEARING

IINI I IFTII

DANODLPH TOWN

$1959 \mathrm{~W}$

\author{
RACKLAND
}

$\begin{array}{rll}1 & 420903 N 2705535.1 & 135 \\ 10 & 42091040705507 . ? & 110 \\ 21 & 42091940705504.1 & 119 \\ 97 & 420014 N 07(5510.1 & 127 \\ 2 & 420910 \times 0705552.1 & 151\end{array}$

UIS GEOL SUPVEY 1967 R

$A B ! N G-R \cap C K$ W BO 1953

ABING-D.ACK 'H BO 1953

$4.20917 \times 0705531.1 \quad 140$

VAVAIR STATION 1954

$\begin{array}{lll}420014 N 0705544.1 & 140 \\ 4 & 147\end{array}$

$\begin{array}{ll}420922 N 57055 ? 1.1 & 126 \\ 420930007 C 5510.1 & 118\end{array}$

NAV AIR STATION 1954

$7 \quad 420924 N 07 C 550^{\circ} .1 \quad 118$

NAV AIO STATIOH 1954

NAV AIP STATION 1954

MAV AIR STATION 1956
YAV AIZ SIATIOY 1956

2. $42072 \div 10705459.1 \quad 15 \mathrm{E}$

NAV AIR STATION $1956 \mathrm{~V}$

$\begin{array}{lll}4 & 42003600705502.1 & 170 \\ 10420020 \times 0705457.1 & 168\end{array}$

$\begin{array}{lll}11 & 42004200705650.1 & 174 \\ 12 & 20916 N 0705551.1 & 152\end{array}$

MAV AIR STATION 1956

YAV AIP STATION 1956

NAV AIP STATION 1956

$14 \quad 420027 \times 0705534.1 \quad 139$

NAV AIF STATION $1954 \mathrm{~V}$

$\begin{array}{ll}4 & - \\ 2 & 0 \\ 2 & 0 \\ 2 & 0 \\ 1 & 0 \\ 1 & 0 \\ 1 & 0 \\ 1 & 0 \\ 1 & 0 \\ 1 & 0 \\ 1 & 0 \\ 1 & 0 \\ 1 & 0 \\ 1 & 0 \\ 1 & 0\end{array}$

\begin{tabular}{|c|c|c|c|}
\hline 27 & $T$ & - & $P k$ \\
\hline 29 & $\dot{r}$ & -- & 78 \\
\hline 41 & $\boldsymbol{r}$ & -- & BR \\
\hline so & $Y$ & -- & -- \\
\hline 14 & $\mathrm{~T}$ & -- & -- \\
\hline 20 & $T$ & - & -. \\
\hline 14 & $\tau$ & -- & -- \\
\hline 16 & $\mathrm{I}$ & -- & -- \\
\hline 25 & $r$ & 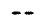 & -- \\
\hline 25 & $\mathrm{~T}$ & -- & -- \\
\hline 6 & $T$ & -- & - \\
\hline 15 & $T$ & -- & -- \\
\hline 11 & $r$ & -- & -- \\
\hline 10 & $T$ & -- & -- \\
\hline 17 & $r$ & -- & -- \\
\hline
\end{tabular}

LEVELIOATE IUSE YIELOI OD ITIME LOG ON (FT) IMEAS-1

(GPM)I(FT)I(HR)

$3420521 \% 0711354.1261$ NOPH

$4 \quad 420623 \times 0711350.1252 \quad$ MOPH

542071900711351.1242 4DPW

$\begin{array}{llll}6 & 420716 \times 0711360.1 & 244 & 42 P_{4} \\ 7 & 420730 N 0711342.1 & 268 & M D P H\end{array}$

$7420526 N 0711354.1248$ 4OPW

R 420630N0711354.1 267 MDPW

9420634 N071135?.1 277 40P

$\begin{array}{llll}10 & 42064 ? 20711357.1 & 219 & 49 P \mathrm{PH} \\ 11 & 42054200711354.1 & 283 & 40 \mathrm{PH}\end{array}$

$12420704: 10711 ? 52.1 \quad 272 \quad 40 \mathrm{PH}$ 42055 An 0710C31.1 300

420554 N0711!31.

$820555 N 0711117.1$
$19 \quad 4205390710957.1$

$20420540.10711014 .1 \quad 260$

$21 \quad 42073310711233.1 \quad 370$

$22 \quad 420709$ N0711133.1 205

33 4207?ON0711350.2 230

$34 \quad 420842.10711155 .1 \quad 212$

$25 \quad 4 ? 0710 N 0711246.1265$

37 420410NOT11355.1 275

$30470717 \times 0711405.1235$

$41 \quad 420 \times 52 N 0711112.1 \quad 210$

$45 \quad 420735 N 0711254.1 \quad 445$

50 420732N0711105.1 220

420854 NOTI1039.

$53 \quad 420358 N 07110 ? ? .1$

55420517 N0711405.1

$5 B$ 420725MO7111?1.1

59
$77 \quad 40725 N 0710842.1$

$78 \quad 420554 N 0711216.1$

$70420650 N 0711113.1$

$8040749 N 0711054.1$

8) $420752 N C 71105 ! 11$

87420715 N0711113.1

$87420531 N 0711025.1$

$101420817 N 0711031.1$

$122420700 N 0711133.2$
RODMAN JOSEPH

EGGEOS RUBY

SORR THCMAS

YORSE DOBERT

WHITE FRFD A

SHAR ON TOHN

NICKERSON T

SP nW' $E$

DE WHUR ST

$\begin{array}{lllr}\text { ROMANSKE } & 1941 & \mathrm{~V} & 2 \\ \text { CRAHLYY HILLIAM } & 1961 & 0 & 42 \\ \text { MFTAL BFILOHS C } & 1962 & \end{array}$

SHARDA TOHN

KENDALL HRS

SHAFON TOWN

SHARON TDWN
SHARON MEY PARK

KNOLLWOND CEM

KULEBABA JOHN

HNHE HILFORD H

HEALEY GEORGE
SHARON TOWN

SHARON TOWN

SHARDN TRHN

SHARON TOWN

SHARON TOHN

SHARON TOWN

SHARON TOWN

SHARON TOWN

SHARON TOHN

SHARON TOHN

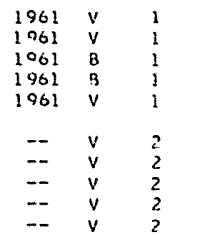

$\begin{array}{rrr}-- & v & 2 \\ -- & v & 2 \\ -- & 0 & -2 \\ -- & v & 2\end{array}$

- 024

$\begin{array}{ccc}-- & v & 1 \\ -- & 0 & 34 \\ 1905 & v & 2\end{array}$

$\begin{array}{ccc}-- & 0 & 12 \\ 1949 & 0 & 30\end{array}$

1899

1962 A

1945

$1957-0) \quad 10$

$\begin{array}{lll}1885 & 0 & 108 \\ 1951 & - & 12\end{array}$

$1950=$

$1954 \quad C$

1964

1966

$1966 \mathrm{H}$

$1966 \mathrm{H}$

$1966 \mathrm{~W}$

1966

1966

1966

1967
$195 !$

20

$\begin{array}{rrrrr}61 & 1 & 61 & 25 & 11 \\ 64 & 1 & -1 & 25 & 0 \\ 32 & 1 & 34 & 5 & 0 \\ 35 & T & 27 & 25 & 8 \\ 74 & T & - & \text { B } & 39\end{array}$

$\begin{array}{llll}32 & \mathrm{~T} & -- & -- \\ 26 & \mathrm{~T} & -- & -- \\ 32 & \mathrm{~T} & -- & --\end{array}$

$\begin{array}{lllllll}20 & 1 & -- & -- & -\end{array}$

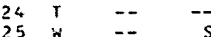

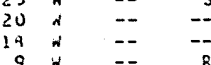

$14 \%$

48
15

$20 \mathrm{~A} \quad--\quad$ U

$\begin{array}{llll}31 & N & -- & \text { UT } \\ 26 & \mathrm{~W} & -- & \text { UR }\end{array}$

$\begin{array}{llll}26 & k & -- & \text { UR } \\ 11 & U & -- & \text { UR }\end{array}$

$\begin{array}{rlll}29 & \mathrm{H} & -1 & \text { UR } \\ 350 & \mathrm{H} & 12 & \end{array}$

$\begin{array}{llll}45 & H & 47 & \text { UP } \\ 22 & U & -- & \text { UT } \\ 86 & H & -- & \text { UR } \\ 18 & 11 & -- & \text { UR }\end{array}$

$\begin{array}{llll}18 & 11 & -- & \text { UR } \\ 43 & 1 & -- & \text { UR }\end{array}$

$57 \quad--\quad$ UR

$\begin{array}{rrrr}209 & w & 14 & --\end{array}$

$\begin{array}{rrrr}150 & H & 9 & -- \\ 39 & \mathrm{~T} & -- & \text { UR }\end{array}$

$\begin{array}{llll}36 & T & -- & \text { UP } \\ 40 & \mathrm{~T} & -- & \text { UP }\end{array}$

$\begin{array}{llll}40 & T & -- & \text { UP } \\ 52 & \mathrm{~T} & -- & \text { UR } \\ 51 & \mathrm{~T} & -- & \text { UR }\end{array}$

$\begin{array}{llll}51 & T & -- & \text { UR } \\ 78 & T & - & \text { UR }\end{array}$

$\begin{array}{llll}18 & T & -- & \text { UR } \\ 38 & \mathrm{~T} & -- & \text { UR }\end{array}$

$\begin{array}{llll}38 & T & -- & \text { UR } \\ 24 & T & -- & \text { UR }\end{array}$

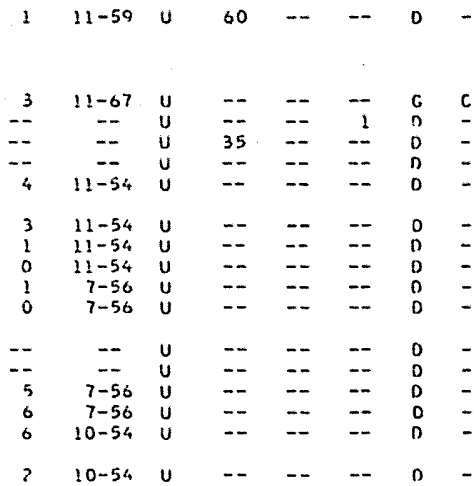

$\begin{array}{llllll}-61 & U & -- & -- & - & 0 \\ -61 & U & -- & -- & -- & 0 \\ -61 & U & -- & -- & -- & 0 \\ -61 & U & -- & -- & -- & 0 \\ -61 & U & -- & -- & -- & 0\end{array}$

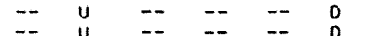

$\begin{array}{llllll}-- & u & -- & -- & -- & 0 \\ -- & u & -- & -- & -- & 0 \\ -- & u & -- & -- & -- & 0 \\ -- & u & -- & -- & -- & 0\end{array}$

$\begin{array}{llllll}-- & U & - & -- & -- & 0\end{array}$

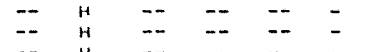

$\begin{array}{llllllllll} & H & - & - & - & - & - & - & - & P\end{array}$

$\begin{array}{lllllll}-- & \text { H } & -- & -- & -- & - & p \\ -- & 4 & -- & -- & -- & - & p\end{array}$

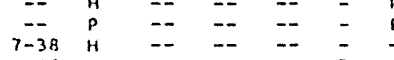

$10 \quad 6-63$

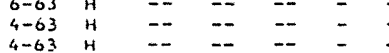

$4-63 \quad H$

$-\quad-20$

$\begin{array}{rrrrrrrr}15 & 9-65 & \text { U } & -- & -- & -- & - & - \\ 3 & 8-57 & p & 1400 & 49 & -- & - & 4 \\ -- & -- & p & -- & -- & - & - & p\end{array}$

$\begin{array}{lllllll}3 & 6-51 & 1 & 232 & 15 & - & -\end{array}$

$\begin{array}{rrrrrrrr}8 & 8-51 & 1 & 505 & 30 & -- & - & - \\ 12 & 3-54 & 4 & 0.5 & -- & -- & - & - \\ 22 & 1-60 & H & 25 & -- & -- & 0 & - \\ 2 & 12-64 & 4 & 0.5 & -- & -- & - & - \\ 0 & 9-66 & 4 & 55 & -- & - & 0 & -\end{array}$

$\begin{array}{lrllllll}2 & 9-66 & u & -- & -- & -- & 0 & - \\ 2 & 10-66 & u & 35 & 4 & 3 & 0 & -\end{array}$

$\begin{array}{llllll}10-66 & U & -- & -- & -- & 0 \\ 10-66 & u & =- & -- & -- & 0\end{array}$

$\begin{array}{lllllll}-\overline{0} & 11-66 & u & -- & -- & -- & \overline{0} \\ 0 & 3-67 & 4 & -- & -- & -- & 0\end{array}$

$\begin{array}{rrrrrrr}0 & 11-66 & U & -- & -- & =- & 0 \\ 6 & 3-67 & U & -- & -- & -- & 0 \\ 2 & 3-67 & U & 50 & =- & -- & 0 \\ 3 & -51 & \mathrm{p} & -- & -- & -- & -\end{array}$

STOUGHTON

* $97 \quad 420740 N 0710817.1 \quad 130$

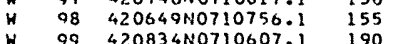

$\begin{array}{rll}499 & 420834 N 0710607.1 & 190 \\ 112 & 42093 B N 0710610.1 & 195 \\ 112 & 420649 N 0710758.1 & 155\end{array}$

W $113 \quad 420654 N 0710748.1 \quad 155$

$W 115$ 42070INOT10752.1

158
180

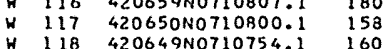

STOUGHTON TOHN
STOUGHTON TOWN
CANTON TOHN
CANTON TOHN
STOUGHTON TOWN
STOUGHTON TOWN
STOUGHTON TOHN
STOUGHTON TOWN
STOUGHTON TOWN
STOUGHTON TOWN

$\begin{array}{llll}1949 & H & 2 & - \\ 1892 & D & -- & H \\ 1894 & D & -- & - \\ 1949 & - & 24 & G \\ 1965 & H & 2 & P \\ 1965 & H & 2 & P \\ 1965 & H & 2 & P \\ 1965 & H & 2 & P \\ 1965 & W & 2 & P \\ 1965 & H & 2 & P\end{array}$

$\begin{array}{llll}62 & W & -- & - \\ -- & W & -- & - \\ -- & W & -- & \text { UR } \\ 43 & W & -- & \text { UR } \\ 37 & T & -- & \text { UR } \\ 33 & T & -- & R \\ 22 & T & -- & -- \\ 64 & T & -- & -- \\ 68 & T & -- & \text { UR } \\ 45 & T & -- & \text { UR }\end{array}$

$\begin{array}{cccccccc}4 & -- & P & 10 & -- & -- & - & M \\ -- & -- & P & 1150 & -- & -- & - & M \\ -- & -- & P & -- & -- & -- & - & M \\ -- & -- & P & -- & -- & -- & - & P \\ 2 & 3-65 & U & 30 & 3 & 4 & D & - \\ 1 & 3-65 & U & 45 & -- & 2 & 0 & - \\ -- & -- & U & -- & -- & -- & - & - \\ - & -- & U & -- & -- & -- & D & - \\ 1 & 3-65 & U & -- & -- & - & D & - \\ 1 & 3-65 & U & 56 & 3 & 2 & 0 & -\end{array}$ 


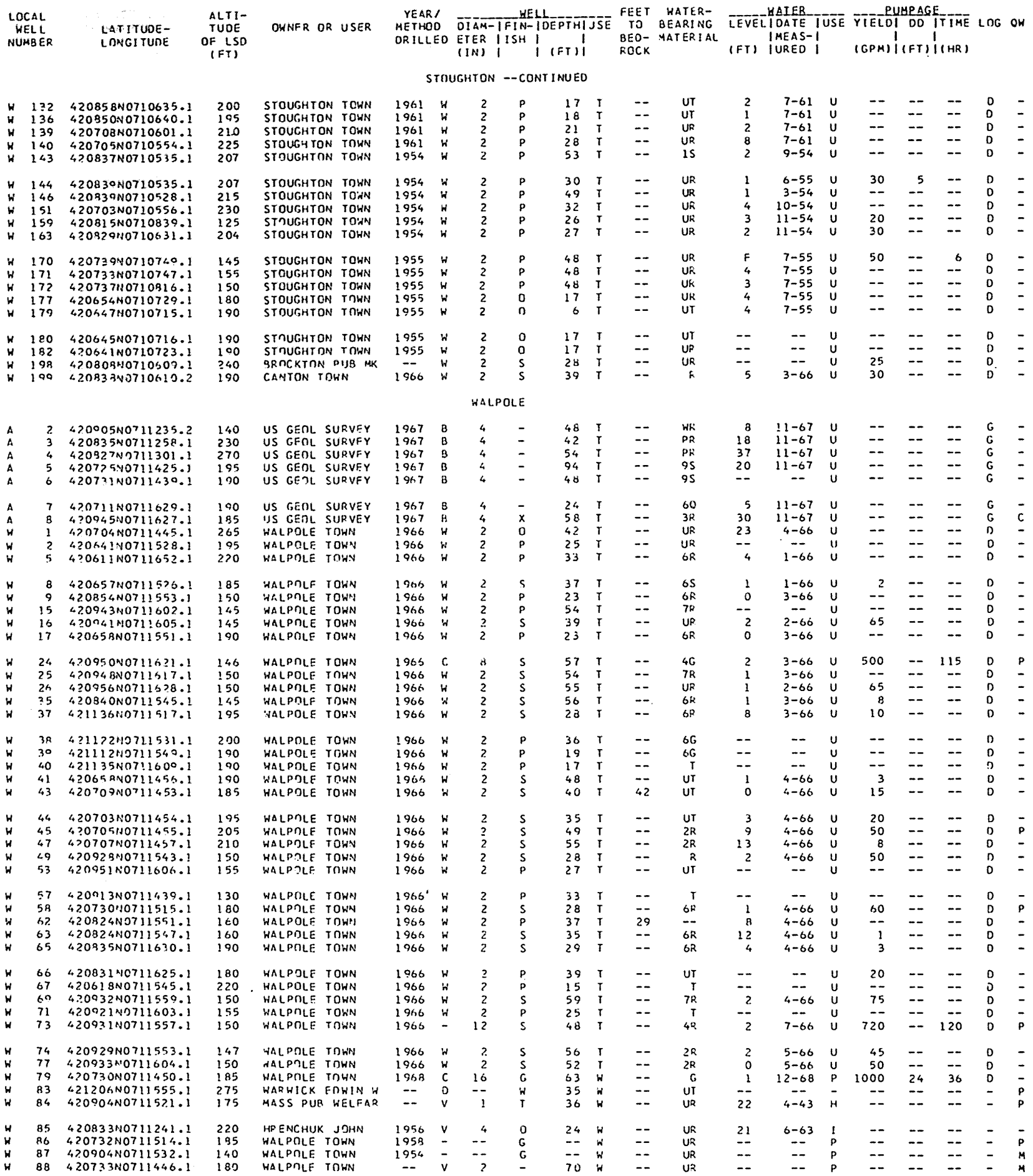




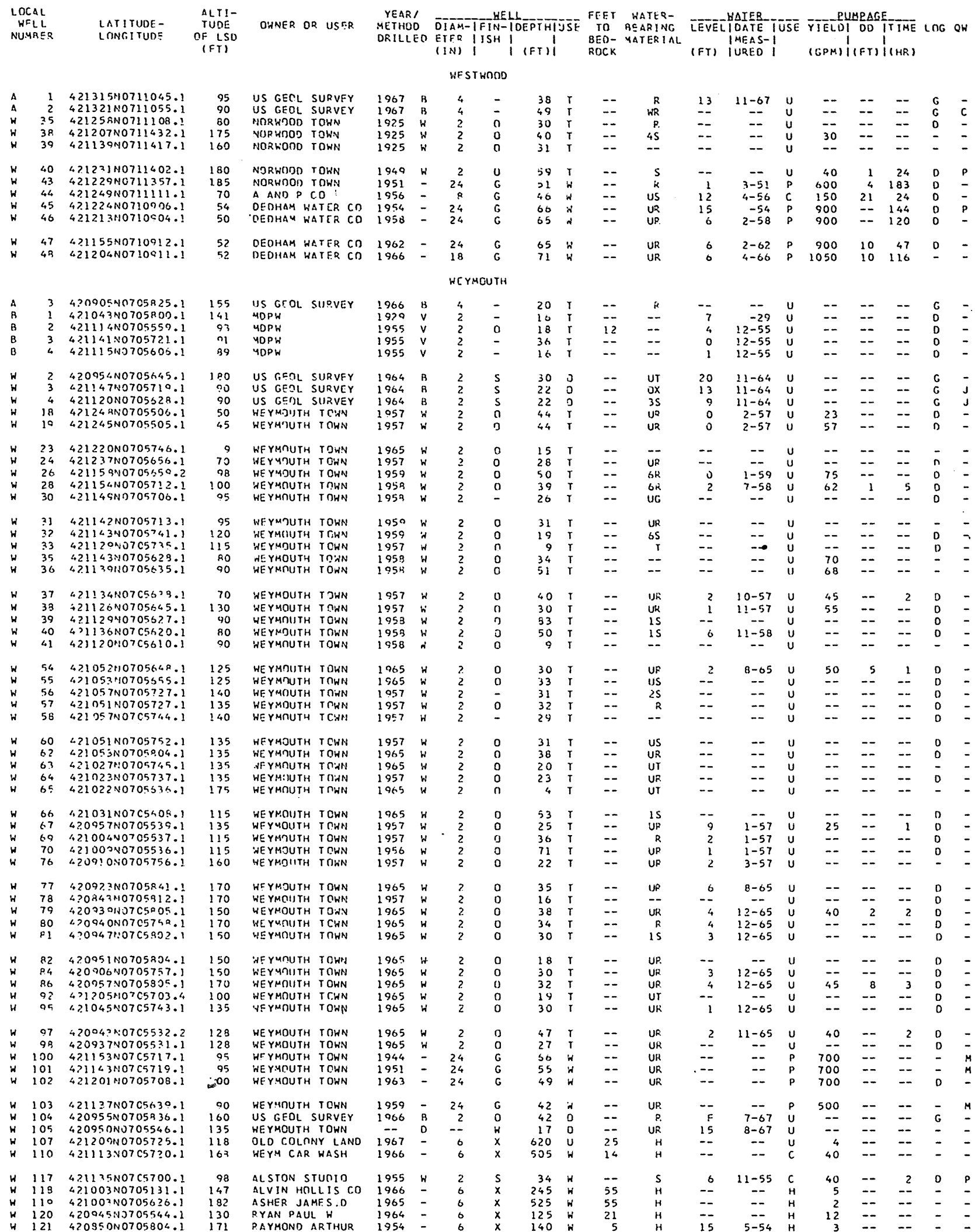


TABLE 1.--DESCRIPTION OF SELECTEO WELLS ANO BORINGS -- CONTINUEO

\begin{tabular}{|c|c|c|c|c|c|c|c|c|c|c|c|c|c|c|c|c|c|c|c|c|c|}
\hline \multicolumn{2}{|c|}{$\begin{array}{l}\text { LOCAL } \\
\text { WFLL } \\
\text { NUMBER }\end{array}$} & \multirow[t]{2}{*}{$\begin{array}{l}\text { LATITUDE- } \\
\text { LONGITUDE }\end{array}$} & \multirow[t]{2}{*}{$\begin{array}{l}\text { ALTI- } \\
\text { TUDE } \\
\text { OF LSO } \\
\text { (FT) }\end{array}$} & \multirow[t]{2}{*}{ OWNER O } & \multirow[t]{2}{*}{ OR USER } & \multicolumn{2}{|c|}{$\begin{array}{l}\text { YEAR/ } \\
\text { METHOD } \\
\text { DRILLED }\end{array}$} & \multicolumn{4}{|c|}{$\begin{array}{l}\text { OIAH-IFIN-IDEPTHIUSE } \\
\text { EIER ISH I IFTI } \\
\text { (IN) I I IFII }\end{array}$} & \multirow[t]{2}{*}{$\begin{array}{l}\text { FEET } \\
\text { TO } \\
B E D- \\
\text { ROCK }\end{array}$} & \multirow[t]{2}{*}{$\begin{array}{l}\text { WATER- } \\
\text { BEARING } \\
\text { HATERIAL }\end{array}$} & \multirow[t]{2}{*}{$\begin{array}{l}\text { LEVEL } \\
\text { (FT) }\end{array}$} & \multicolumn{2}{|c|}{$\begin{array}{l}\text { WAIEB } \\
\text { LOATE IUSE } \\
\text { IHEAS-| } \\
\text { IURED I }\end{array}$} & $\begin{array}{l}\overline{Y I E L D} \\
\text { (GPH) }\end{array}$ & $\begin{array}{l}\text { JPPAC } \\
\text { DO }\end{array}$ & ITIME & \multirow[t]{2}{*}{$\operatorname{LOG}$} & \multirow[t]{2}{*}{ or } \\
\hline \multicolumn{13}{|c|}{ HEYMOUTH - CONTINUED } & & & & & & & & & \\
\hline H & $\begin{array}{l}123 \\
124\end{array}$ & $\begin{array}{l}420934 N 0705615.1 \\
420935 N 0705612.1\end{array}$ & $\begin{array}{l}161 \\
161\end{array}$ & $\begin{array}{l}\text { NAV AIR } \\
\text { NAV AIR }\end{array}$ & $\begin{array}{l}\text { STATION } \\
\text { STATION }\end{array}$ & $\begin{array}{l}1941 \\
1941\end{array}$ & $\begin{array}{l}c \\
c\end{array}$ & $\begin{array}{l}6 \\
6\end{array}$ & $\begin{array}{l}x \\
x\end{array}$ & $\begin{array}{l}50 \\
34\end{array}$ & T & $\begin{array}{l}21 \\
29\end{array}$ & -- & 10 & $11-41$ & $\stackrel{u}{u}$ & 8 & 23 & 3 & $\begin{array}{l}D \\
D\end{array}$ & - \\
\hline W & 126 & $420931 N 0705602.1$ & 160 & NAV AIR & STATION & 1966 & B & 4 & $x$ & 28 & $\mathrm{~T}$ & 18 & -- & -- & -- & $u$ & -- & -- & -. & 0 & - \\
\hline u & 134 & $421033 \times 07 C 5605.1$ & 110 & US GFOL & SURVEY & 1967 & 8 & 2 & $\hat{s}$ & 47 & 0 & -- & PR & 2 & $12-67$ & u & -- & -- & -- & G & - \\
\hline x & 7 & $420947 N 0705705.1$ & 152 & NAV AIR & STATION & 1951 & $H$ & -- & 0 & 30 & $T$ & - & -- & 2 & $3-51$ & $u$ & -- & -- & -- & D & - \\
\hline & 8 & $420950 \mathrm{~N} 0705709.1$ & 15 & NAY AIR & STATION & 95 & $n$ & -- & 0 & 22 & $T$ & -- & -- & 1 & $3-51$ & u & - & -- & - & D & - \\
\hline & 16 & $420955 N 0705636.1$ & 180 & NAV AIR & STATION & 1968 & W & 2 & 0 & 25 & $\mathbf{r}$ & -- & -- & 4 & $5-68$ & $u$ & -- & -- & -- & D & - \\
\hline & 17 & $420948 N 07 C 5620.1$ & 174 & NAV AIR & STATION & 1968 & $H$ & 2 & 0 & 8 & $\mathrm{r}$ & -- & -- & 2 & $5-68$ & $u$ & -- & -- & -- & o & - \\
\hline & 18 & $420940 N 0705609.1$ & 165 & NAV $A I R$ & STATION & 1968 & $w$ & 2 & 0 & 8 & $r$ & -- & -- & 1 & $5-68$ & $u$ & -- & -- & -- & D & - \\
\hline$x$ & 19 & $420938 \times 0705623.1$ & 176 & NAV AIR & STATION & 1968 & $w$ & 2 & 0 & 15 & $\mathrm{~T}$ & -- & -- & 6 & $5-68$ & $u$ & -- & -- & -- & o & - \\
\hline
\end{tabular}


Trble 2.--Logs of selected vells and borings

(Depths are given in feet belov land surface.)

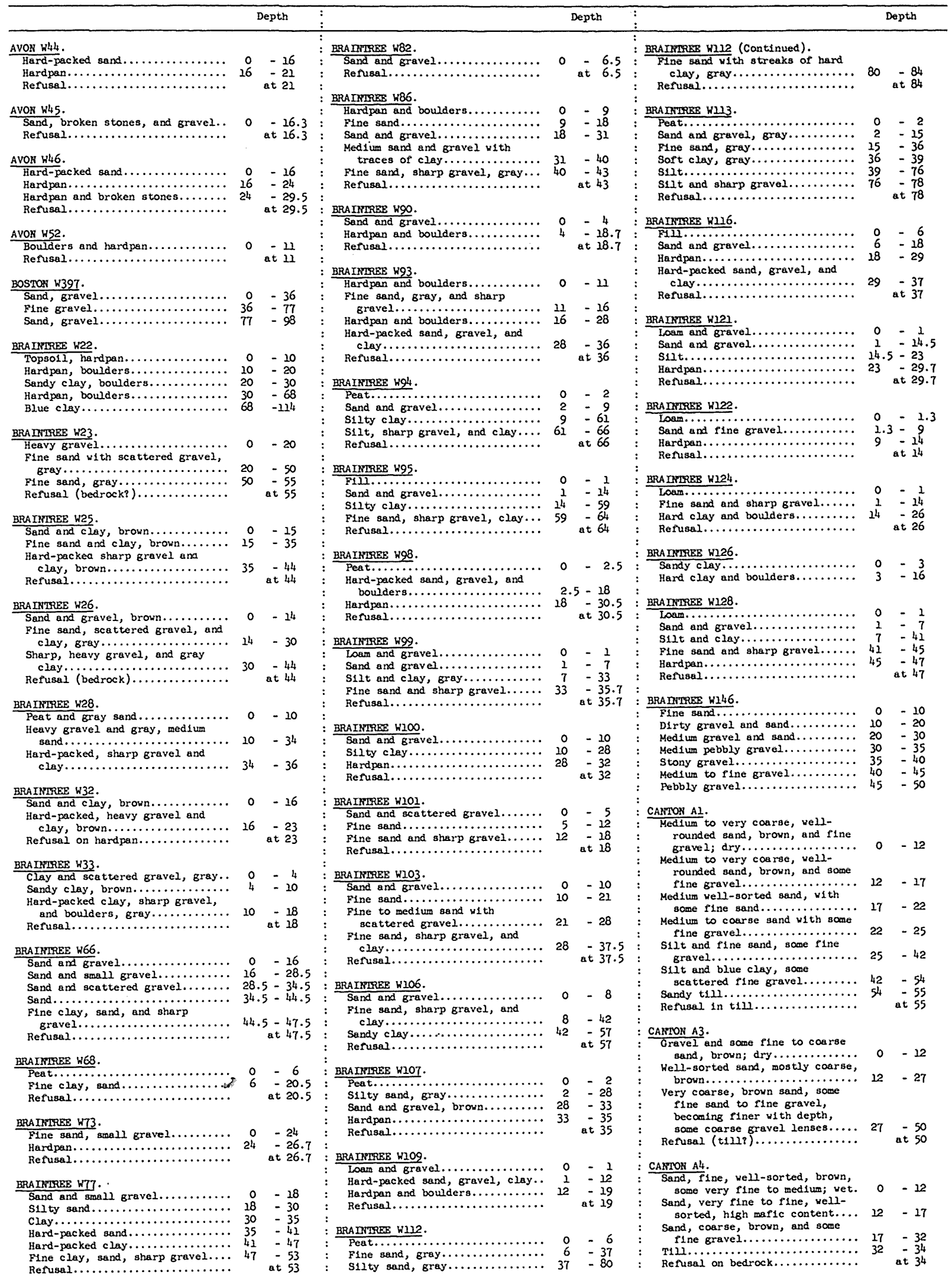


Table 2.--Logs or selected vells and borings (Continued)

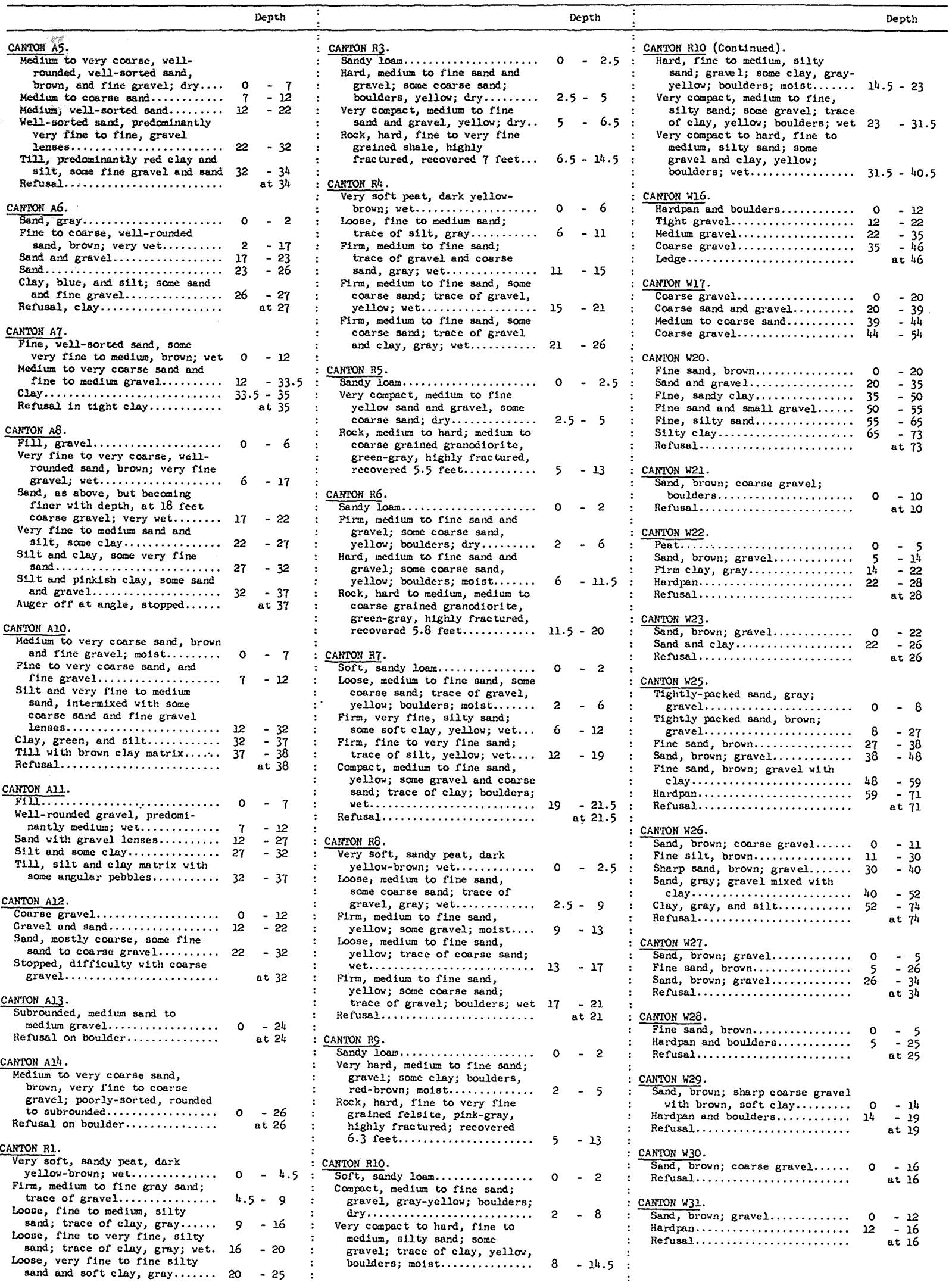


Table 2.--Logs of selected vells and borings (Continued)

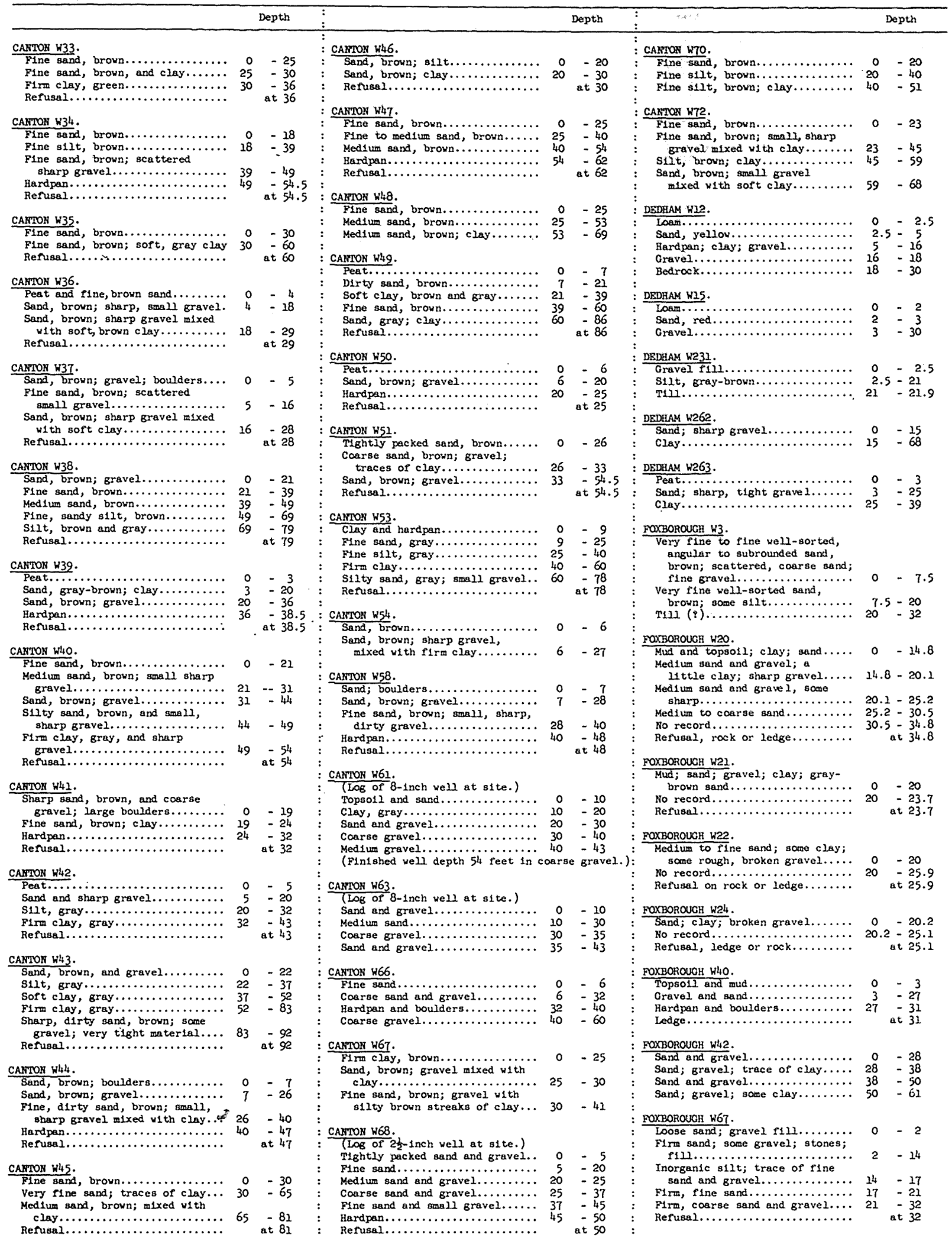


mable 2.--Logs of selected vells and borings (Continued)

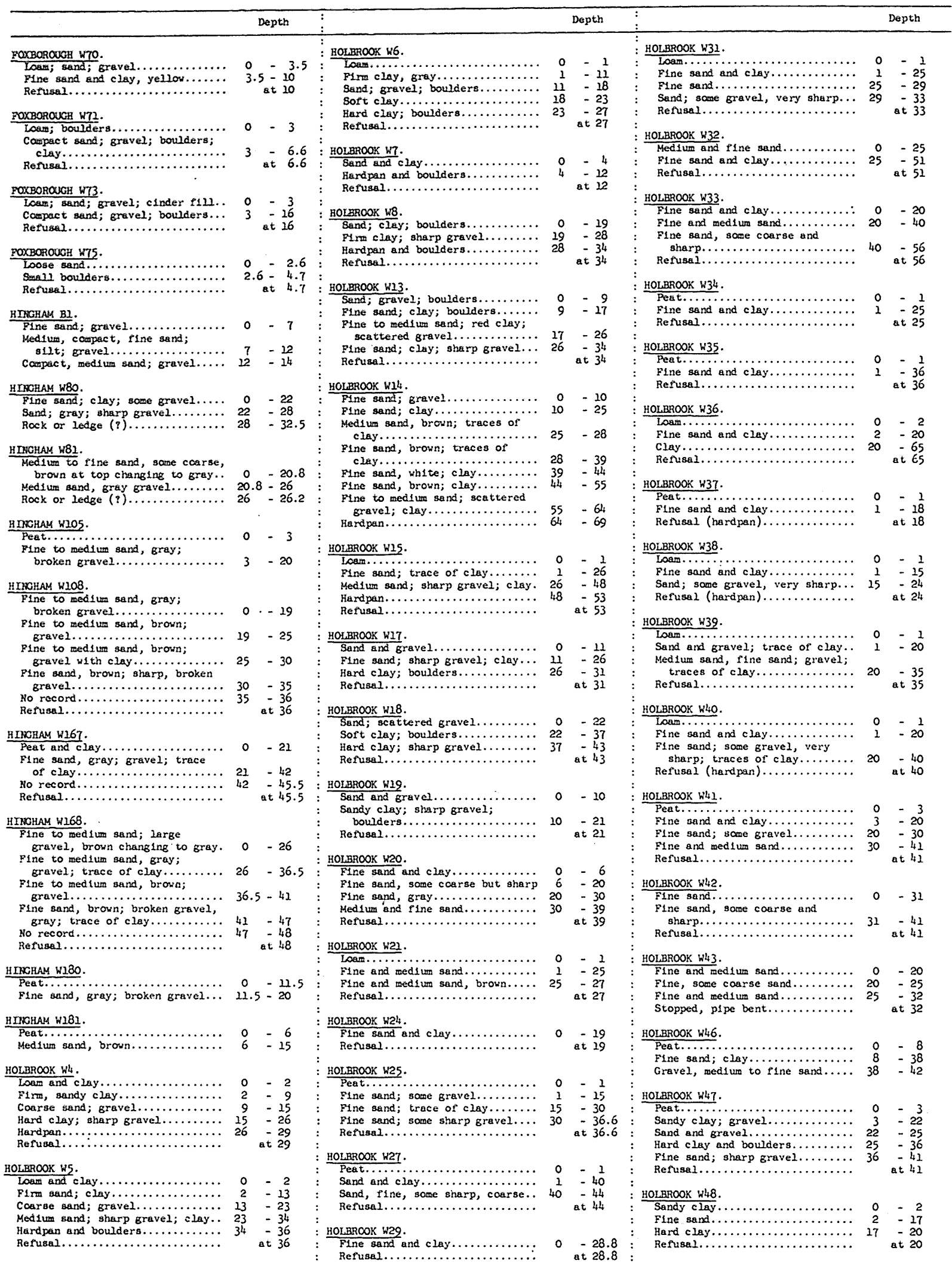


Table 2.--Logs of selected vells and borings (Contimued)

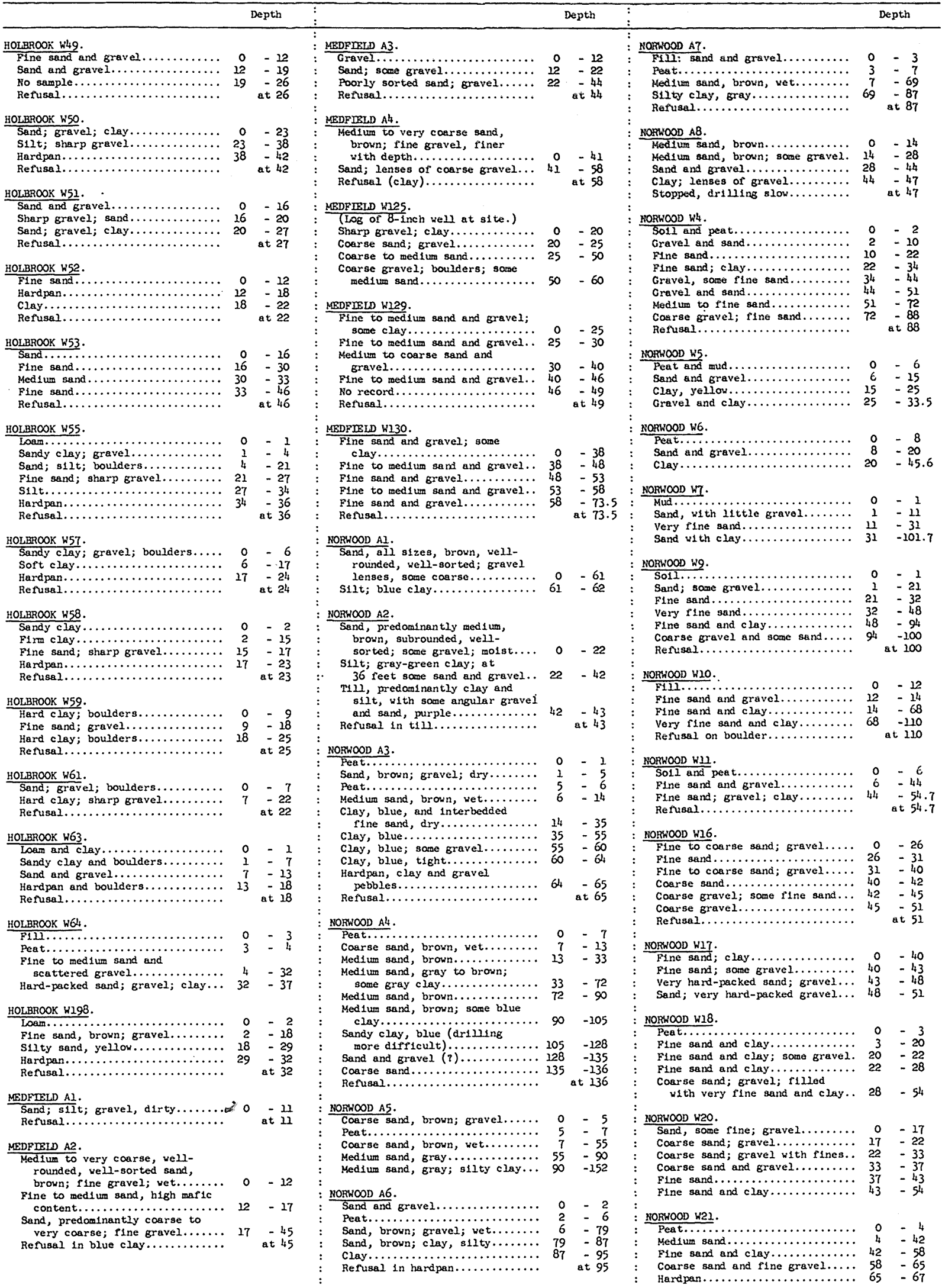


Table 2.--Lags of selected vells and borings (Continued)

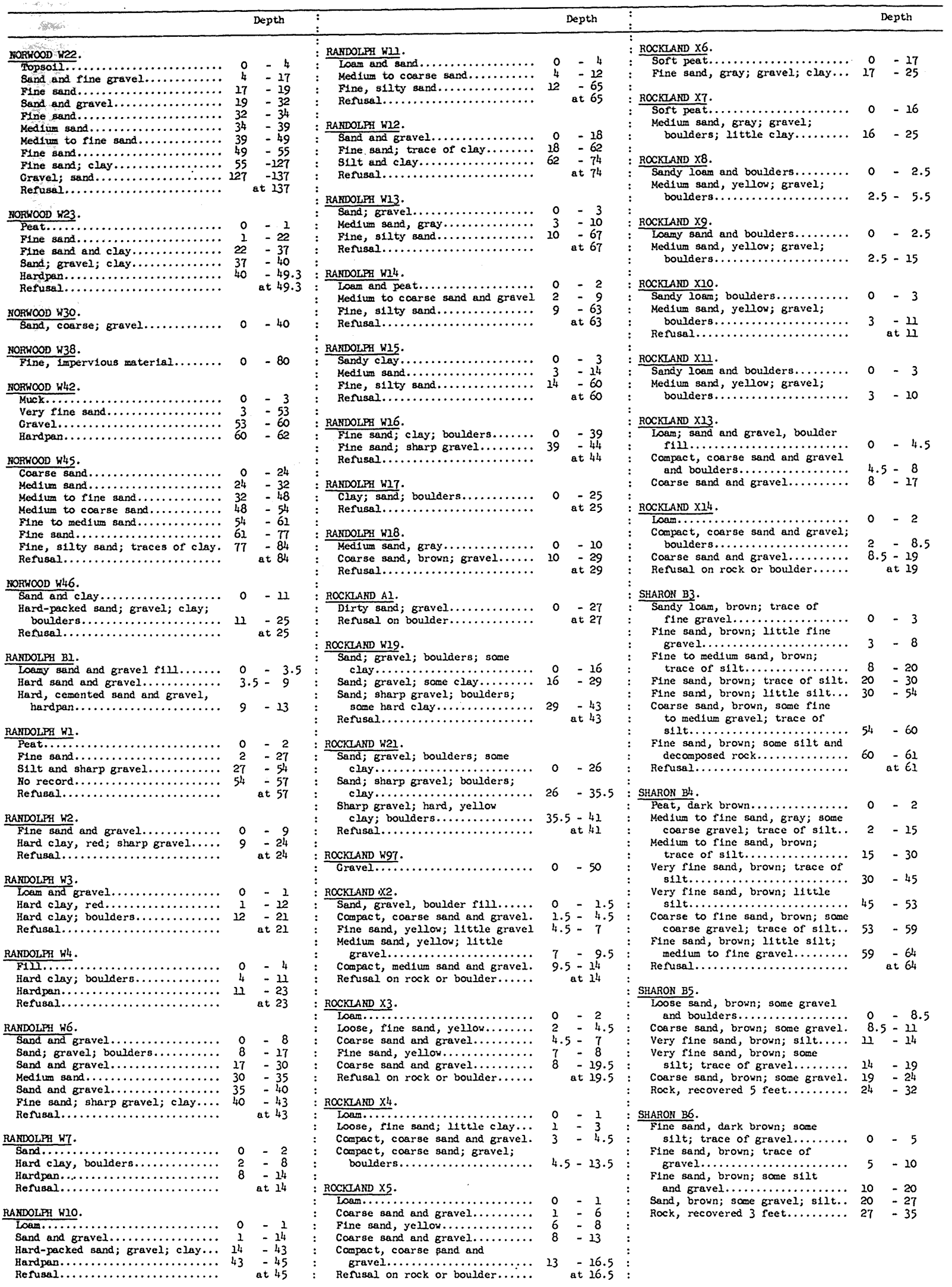


Table 2.--Logs of selected wejls and borings (Continued)

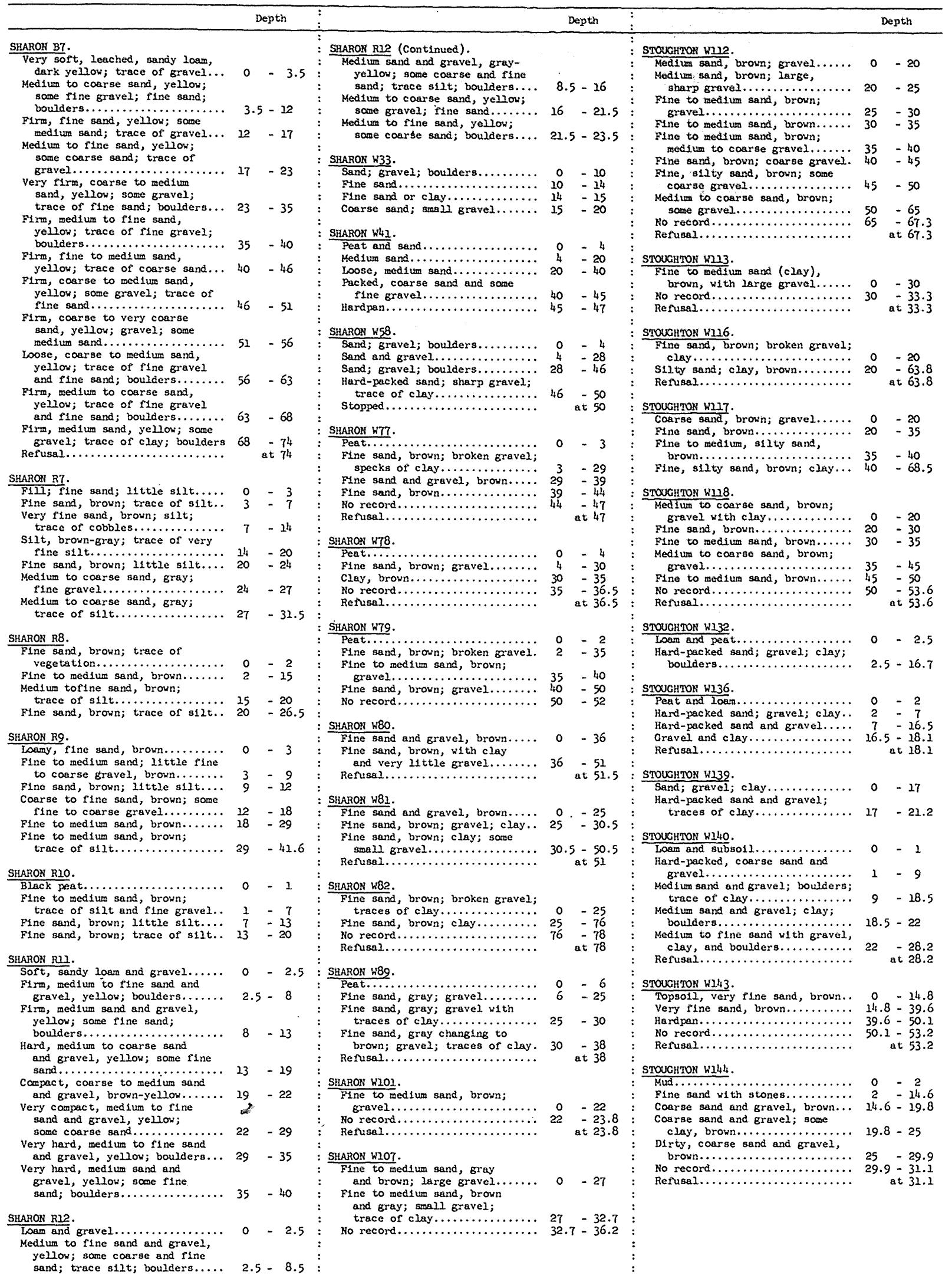


Tuble 2.--Logs or selected vells and borings (Continued)

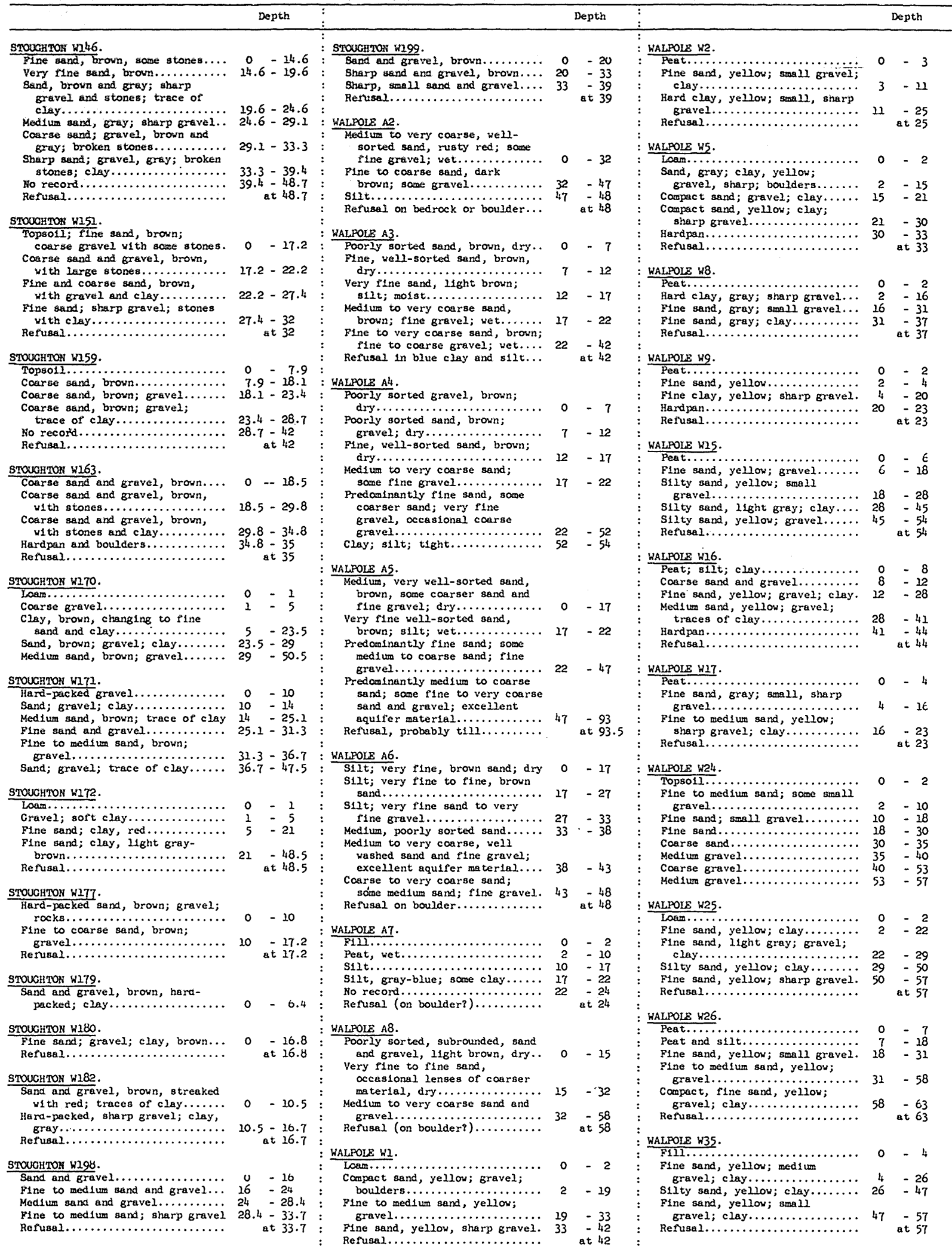


Table 2.--Logs af selected vells and borings (Continued)

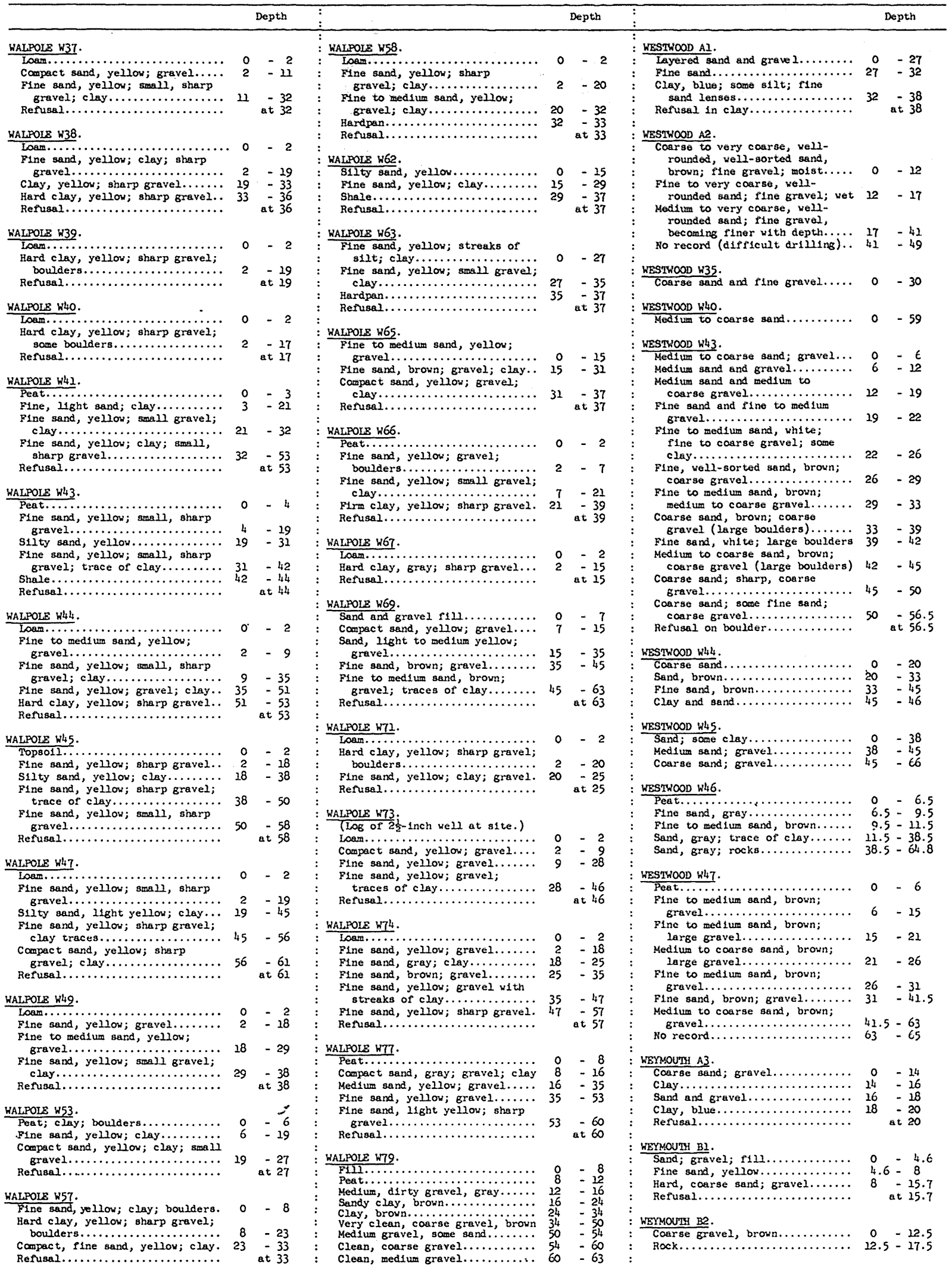


Table 2.--Lags of selected wells and borings (Contlnued)

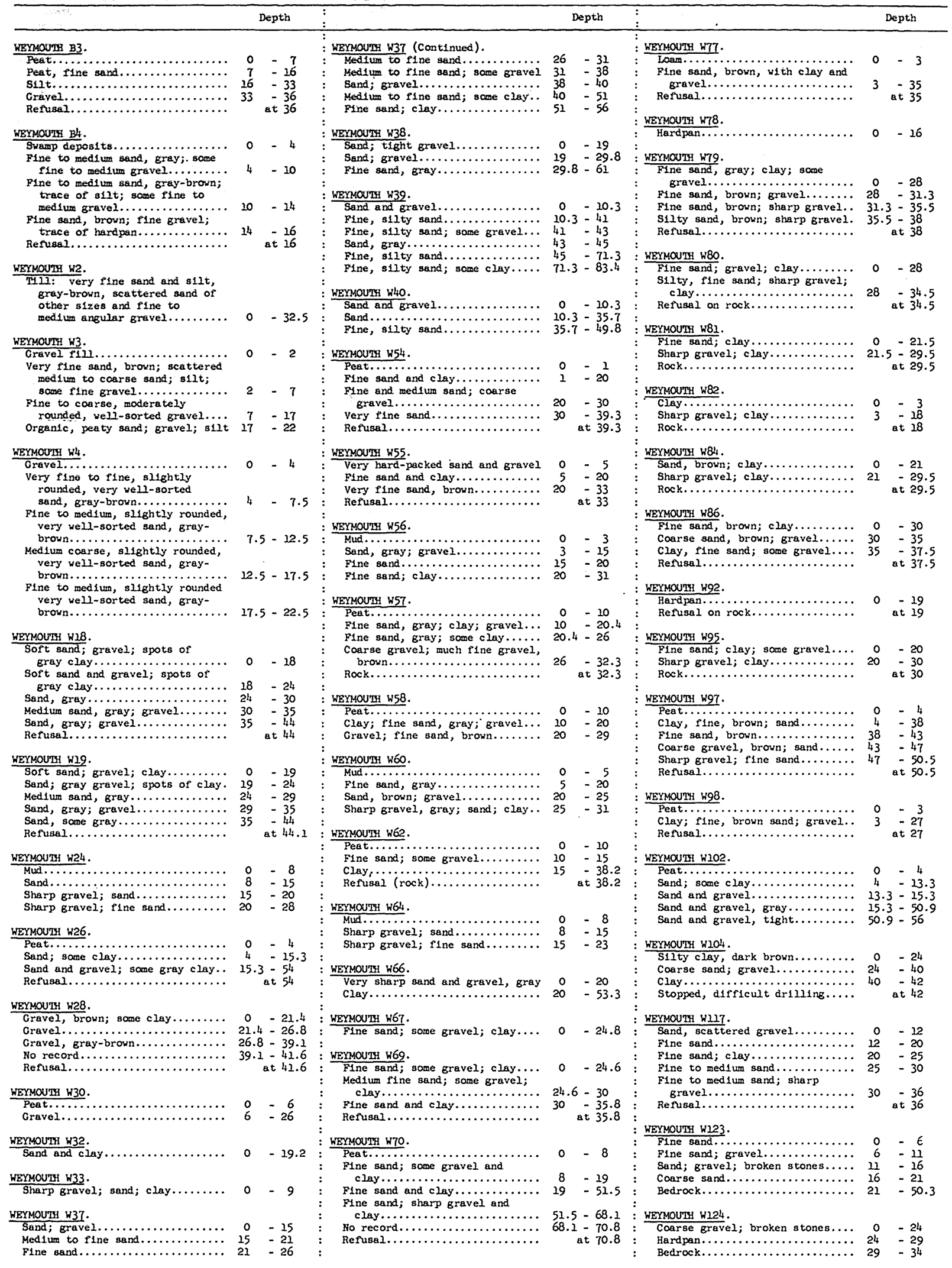


Pable 2.--logs of selected vells and borings (Continued)

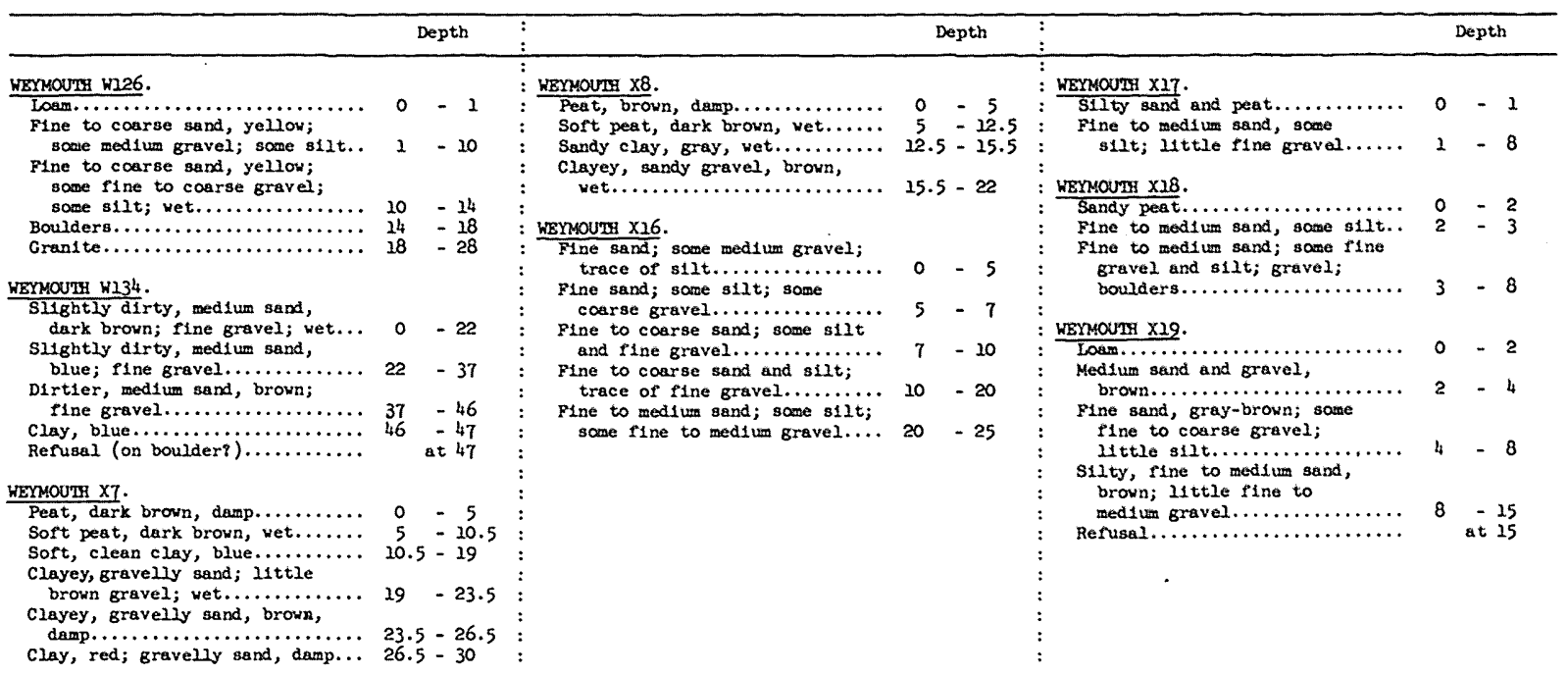


TABIE 3. CHEMICAL, ANALYSES OF GROUND hateR

SOURCE GF DATA: 1, U.S. GE.JLOS,ICAL SURVEY: 2, U.S. PUBLIC HEALTH SERVICE; 3, STATE HEALTH DEPARTMENT:

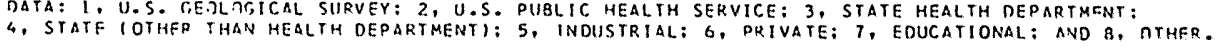

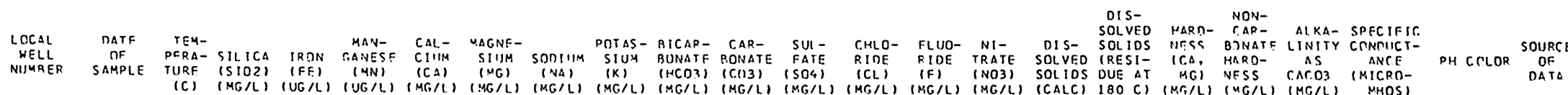

$$
\text { BOAINTREE }
$$

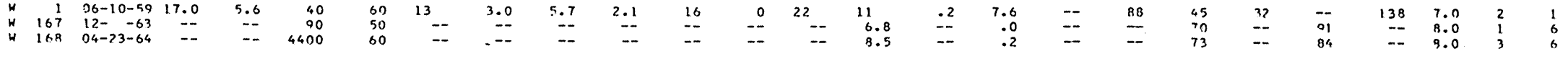

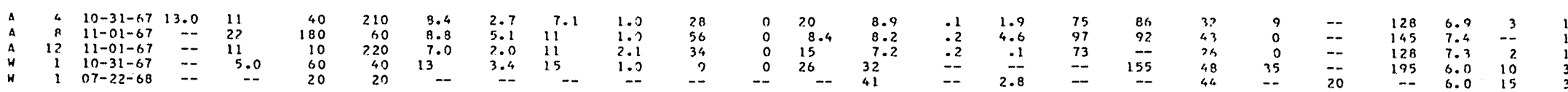

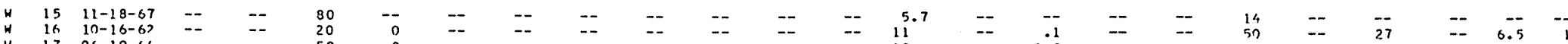

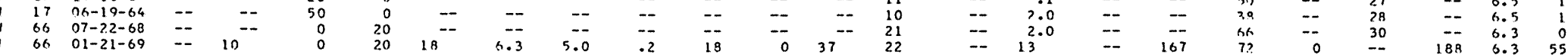

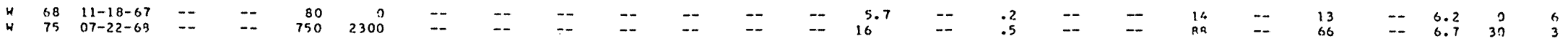

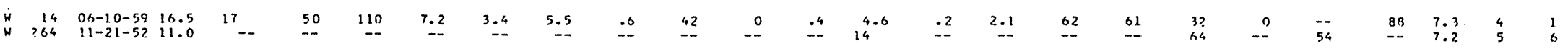

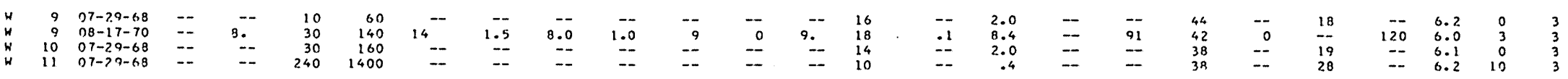

HINGHAM

$\begin{array}{llllllllllllllllllllllllllll}47 & 09-14-67 & -- & 12 & 10 & 160 & 11 & 3.0 & 12 & .9 & 24 & 0 & 14 & 28 & .1 & .2 & 93 & 102 & 40 & 20 & -- & 168 & 6.7 & 0 & 1\end{array}$

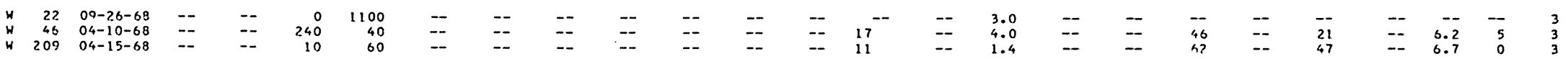

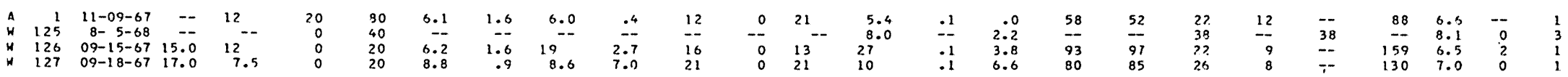


DIS-
SOLVED HARD- TOAR-
T.ALKA- SPECIFIC

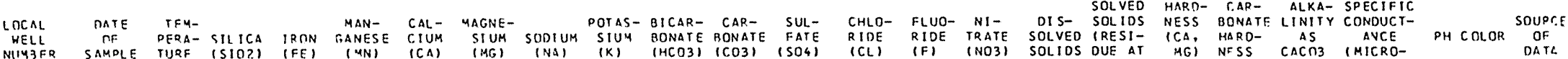

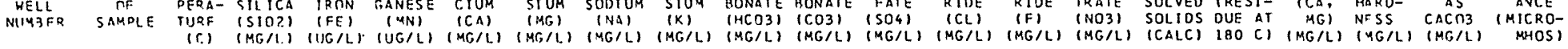

$$
\begin{aligned}
& \text { NORWOOD }
\end{aligned}
$$

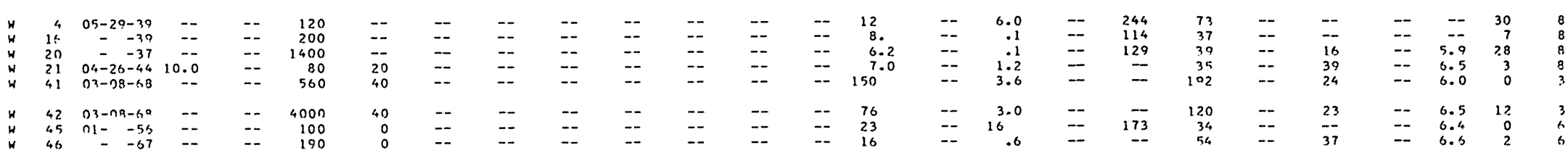

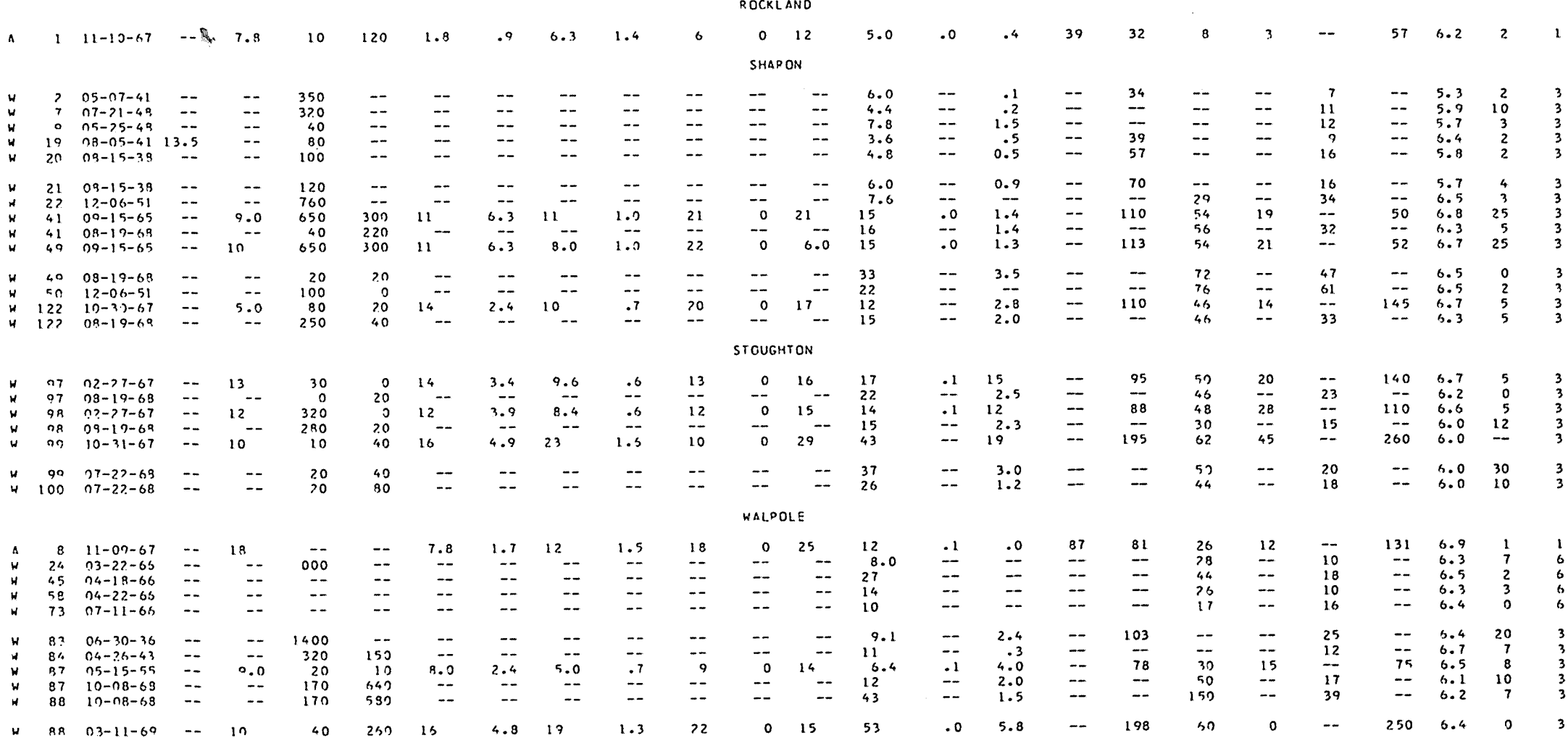


TLBLF 3. ThEMICAI ANAT YSES OF G,POUNI) WATER--CONTINIEE

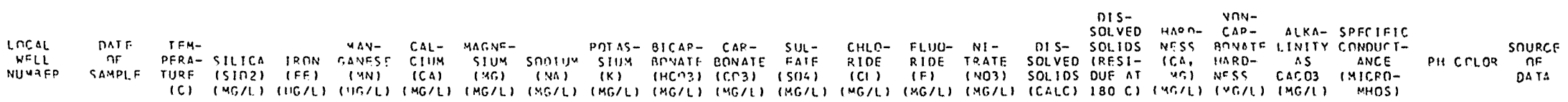
WFSTHOก⿻

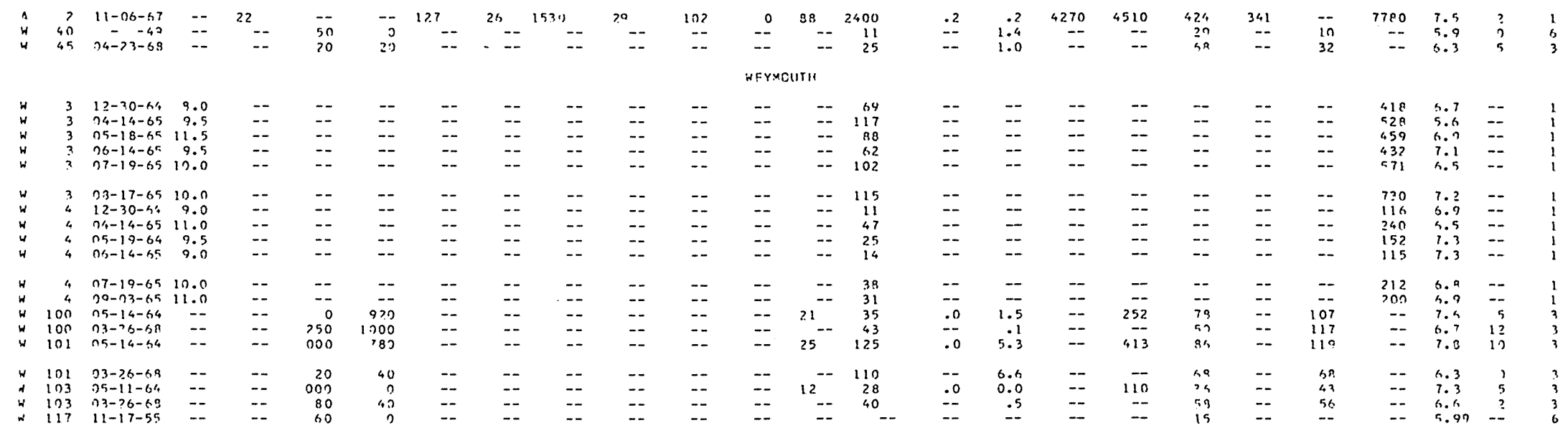


Table 4.--Stream sites and discharge measurements

\begin{tabular}{|c|c|c|c|c|c|}
\hline$\underset{\text { number }}{\text { Map }}$ & $\begin{array}{c}\text { Station name and } \\
\text { U.S. Geological Survey } \\
\text { number }\end{array}$ & Location & $\begin{array}{l}\text { Drainage } \\
\text { area } \\
(\mathrm{sq} \mathrm{m} 1 \text { ) } \\
\end{array}$ & Date. & $\begin{array}{l}\text { Discharge } \\
\text { (cubic feet } \\
\text { per second) }\end{array}$ \\
\hline \multicolumn{6}{|c|}{ NEPONSET RIVER BASIN } \\
\hline 1 & Neponset River & $\begin{array}{l}\text { Lat } 42^{\circ} 05^{\prime} 08^{\prime \prime}, \text { long } 71^{\circ} 15^{\prime} 25^{\prime \prime} \text {, } \\
\text { at North St., } 1.5 \text { miles north } \\
\text { of Foxborough. }\end{array}$ & 1.92 & $\begin{array}{r}10-6-66 \\
5-1-67 \\
8-17-67 \\
8-21-67 \\
9-6-67 \\
9-20-67\end{array}$ & $\begin{array}{l}0.40 \\
1.14 \\
5.37 \\
3.98 \\
7.52 \\
4.67\end{array}$ \\
\hline 2 & Neponset River & $\begin{array}{l}\text { Lat } 42^{\circ} 07^{\prime} 28^{\prime \prime}, \text { long } 71^{\circ} 15^{\prime} 10^{\prime \prime} \text {, } \\
\text { at South St., } 1.5 \text { miles north } \\
\text { of South Walpole. }\end{array}$ & 7.62 & $\begin{array}{r}10-6-66 \\
5-1-67 \\
9-20-67\end{array}$ & $\begin{array}{l}2.40 \\
13.8 \\
5.65\end{array}$ \\
\hline 3 & $\begin{array}{l}\text { School Meadow Brook } \\
(01104830)\end{array}$ & $\begin{array}{l}\text { Lat } 42^{\circ} 07^{\prime} 32^{\prime \prime} \text {, long } 71^{\circ} 14^{\prime} 47^{\prime \prime} \text {, } \\
\text { at Washington St., } 1.5 \text { miles } \\
\text { south of Walpole. }\end{array}$ & 2.80 & $\begin{array}{r}8-16-66 \\
9-12-66 \\
10-6-66 \\
5-1-67 \\
8-17-67 \\
8-21-67 \\
9-6-67 \\
9-20-67\end{array}$ & $\begin{array}{l}.11 \\
.06 \\
.66 \\
5.94 \\
1.57 \\
1.06 \\
.96 \\
.64\end{array}$ \\
\hline 4 & $\begin{array}{l}\text { Neponset River } \\
(01104840)\end{array}$ & $\begin{array}{l}\text { Lat } 42^{\circ} 08^{\prime} 28^{\prime \prime} \text {, long } 71^{\circ} 15^{\prime} 25^{\prime \prime} \text {, } \\
\text { at Main St., Walpole. }\end{array}$ & 11.5 & $\begin{array}{r}10-6-66 \\
12-19-66 \\
1-25-67 \\
3-23-67 \\
5-2-67 \\
6-7-67 \\
7-6-67 \\
8-10-67 \\
8-17-67 \\
8-21-67 \\
9-6-67 \\
9-20-67 \\
10-12-67\end{array}$ & $\begin{array}{c}2.74 \\
8.19 \\
10.6 \\
23.9 \\
21.2 \\
19.7 \\
19.4 \\
15.5 \\
8.82 \\
7.45 \\
31.5 \\
5.96 \\
7.15\end{array}$ \\
\hline 5 & Mine Brook & $\begin{array}{l}\text { Lat } 42^{\circ} 10^{\prime} 58^{\prime \prime}, \text { long } 71^{\circ} 16^{\prime} 58^{\prime \prime} \text {, } \\
\text { at Philip St., } 1.25 \text { miles east } \\
\text { of Medfield. }\end{array}$ & 3.54 & $\begin{array}{r}6-30-67 \\
7-11-67 \\
7-26-67 \\
8-2-67 \\
8-17-67 \\
8-21-67 \\
9-6-67 \\
9-20-67 \\
10-16-67 \\
11-29-67 \\
12-21-67 \\
3-19-68 \\
4-23-68 \\
5-21-68 \\
7-2-68 \\
7-18-68 \\
8-19-68 \\
9-24-68 \\
10-18-68\end{array}$ & $\begin{array}{r}6.00 \\
3.70 \\
2.85 \\
1.99 \\
1.77 \\
1.28 \\
1.48 \\
1.12 \\
1.51 \\
2.79 \\
5.10 \\
133 \\
5.43 \\
7.10 \\
5.35 \\
2.24 \\
.43 \\
.41 \\
.41\end{array}$ \\
\hline 6 & $\begin{array}{l}\text { Mine Brook } \\
(01104850)\end{array}$ & $\begin{array}{l}\text { Lat } 42^{\circ} 09^{\prime} 14^{\prime \prime} \text {, long } 71^{\circ} 15^{\prime} 52^{\prime \prime} \text {, } \\
\text { at 1nlet to Turner Pond, . } 75 \text { mile } \\
\text { northwest of Walpole. Gaging } \\
\text { station data available for period } \\
\text { June } 27 \text {, } 1967 \text {, to July } 31,1968 \\
\text { (see U.S. Geological Survey, 1968). }\end{array}$ & 5.98 & $\begin{array}{r}8-16-66 \\
9-12-66 \\
10-6-66 \\
5-1-67 \\
8-19-68 \\
8-20-68 \\
8-22-68 \\
8-27-68 \\
9-20-68 \\
9-24-68 \\
10-17-68\end{array}$ & $\begin{array}{c}.81 \\
.79 \\
1.28 \\
19.7 \\
.26 \\
.18 \\
.03 \\
0 \\
\text { trace } \\
\text { trace } \\
0\end{array}$ \\
\hline 7 & $\begin{array}{l}\text { Spring Brook } \\
\text { (01104860) }\end{array}$ & $\begin{array}{l}\text { Lat } 42^{\circ} 08^{\prime} 47^{\prime \prime} \text {, long } 71^{\circ} 14^{\prime} 59^{\prime \prime} \text {, } \\
200 \text { feet below outlet of } \\
\text { Memorial Pond, at Walpole. }\end{array}$ & 1.84 & $\begin{array}{l}8-16-66 \\
9-12-66 \\
5-1-67 \\
8-17-67 \\
8-21-67 \\
9-6-67 \\
9-20-67\end{array}$ & $\begin{array}{r}.58 \\
.96 \\
2.77 \\
1.47 \\
.79 \\
1.68 \\
.98\end{array}$ \\
\hline 8 & $\begin{array}{l}\text { Neponset River tributary } \\
(01104880)\end{array}$ & $\begin{array}{l}\text { Lat } 42^{\circ} 09^{\prime} 55^{\prime \prime}, \text { long } 71^{\circ} 14^{\prime} 5^{\prime \prime} \text {, } \\
\text { at Gould st., } 1.33 \text { miles north } \\
\text { of Walpole. }\end{array}$ & 1.51 & $\begin{array}{l}8-16-66 \\
9-12-66 \\
9-14-66 \\
5-2-67 \\
8-17-67 \\
8-21-67 \\
9-7-67 \\
9-8-67 \\
9-20-67\end{array}$ & $\begin{array}{l}0 \\
0 \\
0 \\
2.74 \\
.17 \\
.16 \\
.11 \\
.11 \\
.02\end{array}$ \\
\hline
\end{tabular}


Table 4.--stream sites and discharge measurements (Continued)

\begin{tabular}{|c|c|c|c|c|c|}
\hline $\begin{array}{c}\text { Map } \\
\text { number }\end{array}$ & $\begin{array}{c}\text { Station name and } \\
\text { 0.S. Ceological Survey } \\
\text { number }\end{array}$ & Location & $\begin{array}{c}\text { Dra inage } \\
\text { area } \\
(\mathrm{sg} \mathrm{mi}) \\
\end{array}$ & Date & $\begin{array}{l}\text { Discharge } \\
\text { (cub1c feet } \\
\text { per second) }\end{array}$ \\
\hline \multicolumn{6}{|c|}{ NBFONSET RIVER BÁSIN (Continued) } \\
\hline 9 & Heponset River & $\begin{array}{l}\text { Lat } 42^{\circ} 09^{\prime} 47^{\prime \prime}, \text { long } 71^{\circ} 12^{\prime} 56^{\prime \prime}, \\
\text { at Washington St., East Walpole. }\end{array}$ & 25.2 & -- & (a) \\
\hline 10 & $\begin{array}{l}\text { Mill Brook } \\
(01104905)\end{array}$ & $\begin{array}{l}\text { Lat } 42^{\circ} 11^{\prime} 22^{\prime \prime}, \text { long } 71^{\circ} 14^{\prime} 24^{\prime \prime} \text {, } \\
500 \text { feet above inlet to } \\
\text { Pettee Pond, } 2 \text { miles southwest } \\
\text { of Westwood. }\end{array}$ & 2.27 & $\begin{array}{l}8-16-66 \\
9-12-66 \\
5-2-67 \\
8-17-67 \\
8-21-67 \\
9-6-67 \\
9-8-67 \\
9-20-67 \\
9-23-68\end{array}$ & $\begin{array}{r}0.44 \\
.29 \\
6.10 \\
1.51 \\
1.15 \\
1.30 \\
.86 \\
.81 \\
.48\end{array}$ \\
\hline 11 & $\begin{array}{l}\text { Bubbling Brook } \\
(01104910)\end{array}$ & $\begin{array}{l}\text { Lat } 42^{\circ} 12^{\prime} 04^{\prime \prime}, \text { long } 71^{\circ} 15^{\prime} 01^{\prime \prime} \text {, } \\
\text { at North St., } 1.5 \text { miles southwest } \\
\text { of Westwood. }\end{array}$ & .21 & $\begin{array}{l}8-16-66 \\
9-12-66 \\
5-2-67 \\
8-17-67 \\
8-21-67 \\
9-6-67 \\
9-20-67 \\
9-23-68\end{array}$ & $\begin{array}{l}.03 \\
.40 \\
.07 \\
.08 \\
.06 \\
.05 \\
.02\end{array}$ \\
\hline 12 & $\begin{array}{l}\text { Bubbling Brook tributary } \\
\text { (01104915) }\end{array}$ & $\begin{array}{l}\text { Lat } 42^{\circ} 11^{\prime} 30^{\prime \prime}, \text { long } 71^{\circ} 14^{\prime} 59^{\prime \prime} \text {, } \\
\text { at North St., } 3 \text { miles north of } \\
\text { Walpole. }\end{array}$ & .75 & $\begin{array}{l}8-16-66 \\
9-12-66 \\
5-2-67 \\
8-17-67 \\
8-21-67 \\
9-7-67 \\
9-20-67 \\
9-23-68\end{array}$ & $\begin{array}{l}\text { b.02 } \\
\mathrm{b} .05 \\
1.89 \\
.30 \\
.20 \\
.18 \\
.10 \\
.03\end{array}$ \\
\hline 13 & Bubbling Brook & $\begin{array}{l}\text { Lat } 42^{\circ} 11^{\prime} 19^{\prime \prime}, \text { long } 71^{\circ} 14^{\prime} 32^{\prime \prime} \text {, } \\
100 \text { feet above inlet to Pettee Pond, } \\
2 \text { miles southwest of Westwood. }\end{array}$ & -- & $\begin{array}{l}9-8-67 \\
9-20-67 \\
9-23-68\end{array}$ & $\begin{array}{r}.27 \\
.09 \\
\text { trace }\end{array}$ \\
\hline 14 & $\begin{array}{l}\text { Willett Pond outlet } \\
\text { (head of Hawes Brook.) } \\
\text { (01204940) }\end{array}$ & $\begin{array}{l}\text { Lat } 42^{\circ} 10^{\prime} 50^{\prime \prime} \text {, long } 77^{\circ} 14^{\prime} \infty 0^{\prime \prime} \text {, } \\
\text { at outlet of Willett Pond, } \\
2 \text { miles southwest of Norwood. }\end{array}$ & 4.90 & $9-28-66$ & 6.98 \\
\hline 15 & $\begin{array}{l}\text { Germany Brook } \\
(01104960)\end{array}$ & $\begin{array}{l}\text { Lat } 42^{\circ} 11^{\prime} 04^{\prime \prime}, \text { long } 71^{\circ} 13^{\prime} 29^{\prime \prime} \text {, } \\
100 \text { feet above culvert on } \\
\text { N1chols St., } 1.3 \text { m1les vest of } \\
\text { Norwood. }\end{array}$ & 2.36 & $\begin{array}{r}8-16-66 \\
9-12-66 \\
9-28-66 \\
12-19-66 \\
1-25-67 \\
3-23-67 \\
5-2-67 \\
6-7-67 \\
7-7-67 \\
8-11-67 \\
8-17-67 \\
8-21-67 \\
9-6-67 \\
9-20-67 \\
9-24-68\end{array}$ & $\begin{array}{r}.17 \\
.14 \\
.34 \\
1.26 \\
1.90 \\
3.84 \\
5.17 \\
2.60 \\
1.79 \\
.30 \\
.56 \\
.41 \\
.43 \\
.32 \\
.20\end{array}$ \\
\hline 16 & $\begin{array}{l}\text { Hawes Brook } \\
(01104980)\end{array}$ & $\begin{array}{l}\text { Lat } 42^{\circ} 10^{\prime} 26^{\prime \prime} \text {, long } 71^{\circ} 12^{\prime} 31^{\prime \prime} \text {, } \\
\text { at Wasbington St., Norwood. }\end{array}$ & 8.65 & $\begin{array}{r}10-6-66 \\
12-19-66 \\
1-25-67 \\
3-23-67 \\
6-7-67 \\
7-7-67 \\
8-11-67 \\
10-12-67\end{array}$ & $\begin{array}{c}.74 \\
2.68 \\
3.98 \\
21.8 \\
11.7 \\
11.6 \\
6.41 \\
2.76\end{array}$ \\
\hline 17 & $\begin{array}{l}\text { Neponset River } \\
\text { (01105000) }\end{array}$ & $\begin{array}{l}\text { Lat } 42^{\circ} 10^{\prime} 39^{\prime \prime} \text {, long } 71^{\circ} 12^{\prime} 05^{\prime \prime} \text {, } \\
200 \text { feet above bridge on } \\
\text { Pleasant St., Norwood. Gaging } \\
\text { station data ava1lable since } 1939 . \\
\text { (See U.S. Geolog1cal Survey, } 1954 \text {, } \\
1964,1966-73 \text {, and } 1969 . \text { ) }\end{array}$ & 35.2 & & \\
\hline 18 & $\begin{array}{l}\text { Traphole Brook } \\
(01105100)\end{array}$ & $\begin{array}{l}\text { Lat } 42^{\circ} 09^{\prime} 36^{\prime \prime}, \text { long } 71^{\circ} 11^{\prime} 47^{\prime \prime} \text {, } \\
\text { at Sumner St., } 2.25 \text { miles south } \\
\text { of Norwood. }\end{array}$ & $3 \cdot 39$ & $\begin{array}{r}7-29-59 \\
8-18-59 \\
9-14-59 \\
9-22-59 \\
7-13-60 \\
8-30-60 \\
8-16-66 \\
9-13-66 \\
10-6-66 \\
5-2-67 \\
7-1-67 \\
7-26-67\end{array}$ & $\begin{array}{l}4.18 \\
2.81 \\
1.82 \\
2.09 \\
1.64 \\
1.88 \\
.97 \\
.92 \\
1.56 \\
6.68 \\
3.29 \\
3.01\end{array}$ \\
\hline
\end{tabular}


Table 4.--Stream sites and discharge measurements (Continued)

\begin{tabular}{|c|c|c|c|c|c|}
\hline $\begin{array}{c}\text { Map } \\
\text { number }\end{array}$ & $\begin{array}{c}\text { Station name and } \\
\text { U.S. Geological Survey } \\
\text { number }\end{array}$ & Location & $\begin{array}{l}\text { Drainage } \\
\text { area } \\
(\mathrm{sq} \mathrm{mi})\end{array}$ & Date & $\begin{array}{l}\text { Discharge } \\
\text { (cubic feet } \\
\text { per second) }\end{array}$ \\
\hline \multicolumn{6}{|c|}{ NEPONSET RTVER BASIN (Continued) } \\
\hline 18 & $\begin{array}{l}\text { Traphole Brook } \\
\text { (01105100).--Continued }\end{array}$ & & & $\begin{array}{r}8-17-67 \\
8-22-67 \\
9-7-67 \\
9-21-67 \\
11-29-67 \\
3-19-68 \\
7-2-68 \\
7-30-68 \\
9-17-68 \\
9-24-68\end{array}$ & $\begin{array}{c}2.02 \\
2.39 \\
1.94 \\
1.62 \\
3.08 \\
46.8 \\
3.49 \\
1.60 \\
1.20 \\
1.34\end{array}$ \\
\hline 19 & $\begin{array}{l}\text { Neponset River } \\
\text { tributary no. } 2 \\
(01105150)\end{array}$ & $\begin{array}{l}\text { Lat } 42^{\circ} 08^{\prime} 52^{\prime \prime} \text {, long } 71^{\circ} 10^{\prime 4} 48^{\prime \prime} \text {, } \\
\text { at Edge Hill Rd., } 1.75 \text { miles } \\
\text { north of Sharon. }\end{array}$ & 0.38 & $\begin{array}{l}8-16-66 \\
9-13-66 \\
5-2-67 \\
8-18-67 \\
8-23-67 \\
9-7-67 \\
9-21-67 \\
7-30-68 \\
9-17-68\end{array}$ & $\begin{array}{r}\text { trace } \\
\text { trace } \\
1.18 \\
.14 \\
.12 \\
.12 \\
.06 \\
.05 \\
\mathrm{~b} .01\end{array}$ \\
\hline 20 & Massapoag Brook & $\begin{array}{l}\text { Lat } 42^{\circ} 06^{\prime} 53^{\prime \prime} \text {, long } 71^{\circ} 10^{\prime} 19^{\prime \prime} \text {, } \\
\text { at Ames St., Sharon. }\end{array}$ & 4.25 & $\begin{array}{r}10-6-66 \\
8-18-67 \\
8-23-67 \\
9-8-67 \\
9-21-67\end{array}$ & $\begin{array}{r}1.73 \\
3.62 \\
1.44 \\
2.76 \\
.73\end{array}$ \\
\hline 21 & Messaparg Brook & $\begin{array}{l}\text { Lat } 42^{\circ} 08^{\prime} 12^{\prime \prime} \text {, long } 72^{\circ} 09^{\prime} 59^{\prime \prime} \text {, } \\
\text { at State Highway } 27,1 \text { mile } \\
\text { northeast of Sharon. }\end{array}$ & 6.32 & $\begin{array}{l}8-16-66 \\
9-13-66 \\
8-18-67 \\
8-23-67 \\
9-8-67 \\
9-21-67\end{array}$ & $\begin{array}{l}.76 \\
1.08 \\
3.60 \\
2.77 \\
2.66 \\
1.63\end{array}$ \\
\hline 22 & $\begin{array}{l}\text { Beaver Brook } \\
\text { (01105255) }\end{array}$ & $\begin{array}{l}\text { Lat } 42^{\circ} 07^{\prime} 59^{\prime \prime}, \text { Iong } 71^{\circ} 10^{\prime} 41^{\prime \prime} \text {, } \\
\text { at Maskwonicut St., } 0.75 \mathrm{mile} \\
\text { north of Sharon. }\end{array}$ & 2.45 & $\begin{array}{l}8-16-6 t \\
9-13-66 \\
5-2-67 \\
8-18-67 \\
8-22-67 \\
9-7-67 \\
9-21-67 \\
9-23-68\end{array}$ & $\begin{array}{l}.59 \\
.59 \\
8.67 \\
2.67 \\
2.31 \\
1.88 \\
1.52 \\
1.31\end{array}$ \\
\hline 23 & $\begin{array}{l}\text { Steep Hill Brook } \\
\text { (01105300) }\end{array}$ & $\begin{array}{l}\text { Lat } 42^{\circ} 08^{\prime} 39^{\prime \prime}, \text { long } 71^{\circ} 08^{\prime} 14^{\prime \prime} \text {, } \\
\text { at Bailey St., I mile southeast } \\
\text { of Canton. }\end{array}$ & 6.66 & $\begin{array}{r}9-13-66 \\
10-6-66 \\
7-12-67 \\
8-2-67 \\
8-18-67 \\
8-23-67 \\
9-7-67 \\
9-21-67 \\
9-23-68\end{array}$ & $\begin{array}{l}2.13 \\
4.42 \\
9.16 \\
8.46 \\
7.77 \\
5.99 \\
5.91 \\
5.34 \\
5.54\end{array}$ \\
\hline 24 & $\begin{array}{l}\text { Redwing Brook } \\
(01105350)\end{array}$ & $\begin{array}{l}\text { Lat } 42^{\circ} 08^{\prime} 59^{\prime \prime}, \text { long } 71^{\circ} 07^{\prime} 36^{\prime \prime} \text {, } \\
\text { at Pleasant St., } 1 \text { mile east } \\
\text { of Canton. }\end{array}$ & 2.45 & $\begin{array}{r}9-13-66 \\
10-7-66 \\
5-2-67 \\
8-18-67 \\
8-23-67 \\
9-7-67 \\
9-21-67\end{array}$ & $\begin{array}{r}.91 \\
1.16 \\
6.17 \\
.87 \\
.22 \\
.66 \\
.18\end{array}$ \\
\hline 25 & $\begin{array}{l}\text { Pequid Brook } \\
(01105400)\end{array}$ & $\begin{array}{l}\text { Lat } 42^{\circ} 10^{\prime} 29^{\prime \prime} \text {, long } 71^{\circ} 06^{\prime} 45^{\prime \prime} \text {, } \\
\text { at State Highway } 138,2 \text { miles } \\
\text { northeast of Canton. }\end{array}$ & $4.5^{4}$ & $\begin{array}{l}9-13-66 \\
5-2-67 \\
8-18-67 \\
8-22-67 \\
9-7-67 \\
9-21-67 \\
9-20-68\end{array}$ & $\begin{array}{r}.73 \\
10.9 \\
1.37 \\
1.03 \\
1.60 \\
.20 \\
\text { trace }\end{array}$ \\
\hline 26 & Pequid Brook & $\begin{array}{l}\text { Lat } 42^{\circ} 10^{\prime} 03^{\prime \prime} \text {, long } 71^{\circ} 08^{\prime} 04^{\prime \prime} \text {, } \\
\text { at Pleasant St., I mile northeast } \\
\text { of Canton. }\end{array}$ & 6.24 & $\begin{array}{r}8-15-66 \\
9-14-66 \\
10-7-66 \\
5-2-67\end{array}$ & $\begin{array}{l}1.79 \\
1.48 \\
1.55 \\
12.8\end{array}$ \\
\hline 27 & $\begin{array}{l}\text { East Branch Neponset } \\
\text { R1ver } \\
\text { (01105500) }\end{array}$ & $\begin{array}{l}\text { Lat } 42^{\circ} 09^{\prime} 16^{\prime \prime} \text {, long } 71^{\circ} 08^{\prime} 47^{\prime \prime} \text {, } \\
100 \text { feet below bridge on } \\
\text { Washington St., Canton. Gaglng } \\
\text { station data available since } \\
\text { October } 1952 \text {. (See U.S. } \\
\text { Geological Survey } 1954 \text {, } 1964 \text {, } \\
1966-73 \text {, and } 1969 .)\end{array}$ & 27.2 & & \\
\hline
\end{tabular}


Table 4.--Stream sites and discharge measurements

\begin{tabular}{|c|c|c|c|c|c|}
\hline $\begin{array}{c}\text { Map } \\
\text { number }\end{array}$ & $\begin{array}{c}\text { Station name and } \\
\text { U.S. Geological Survey } \\
\text { number }\end{array}$ & Location & $\begin{array}{l}\text { Drainage } \\
\text { area } \\
\text { (sq mi) }\end{array}$ & Date & $\begin{array}{l}\text { Discharge } \\
\text { (cublc feet } \\
\text { per second }\end{array}$ \\
\hline \multicolumn{6}{|c|}{ NEPOKSET RIVER BASIN (Continued) } \\
\hline 28 & Purgatory Brook & $\begin{array}{l}\text { Lat } 42^{\circ} 12^{\prime} 54^{\prime \prime} \text {, long } 71^{\circ} 11^{\prime} 24^{\prime \prime} \text {, } \\
\text { at U.S. Highway } 1 \mathrm{~A} \text {, at } \\
\text { Islington. }\end{array}$ & 2.24 & $\begin{array}{l}7-29-59 \\
8-18-59 \\
9-14-59 \\
7-13-60 \\
8-30-60\end{array}$ & $\begin{array}{r}2.03 \\
.27 \\
.07 \\
.08 \\
.01\end{array}$ \\
\hline 29 & $\begin{array}{l}\text { Purgatory Brook } \\
(01105530)\end{array}$ & $\begin{array}{l}\text { Lat } 42^{\circ} 12^{\prime} 33^{\prime \prime} \text {, long } 71^{\circ} 11^{\prime} 06^{\prime \prime} \text {, } \\
\text { at U.S. Highway } 1,1.5 \text { miles } \\
\text { northeast of Norwood. }\end{array}$ & 2.91 & $\begin{array}{l}8-16-66 \\
9-12-66 \\
9-28-66 \\
5-2-67 \\
8-18-67 \\
8-22-67 \\
9-7-67 \\
9-21-67 \\
7-30-68 \\
9-17-68\end{array}$ & $\begin{array}{r}.42 \\
.31 \\
.18 \\
6.41 \\
.89 \\
.76 \\
.71 \\
.40 \\
.85 \\
.64\end{array}$ \\
\hline 30 & $\begin{array}{l}\text { Plantingfield Brook } \\
(01105545)\end{array}$ & $\begin{array}{l}\text { Lat } 42^{\circ} 12^{\prime} 19^{\prime \prime} \text {, long } 71^{\circ} 11^{\prime} 48^{\prime \prime} \text {, } \\
\text { at State Highway } 1 \mathrm{~A}, 0.75 \mathrm{mille} \\
\text { north of Norwood. }\end{array}$ & 1.02 & $\begin{array}{l}8-16-66 \\
9-12-66 \\
5-2-67 \\
8-18-67 \\
8-22-67 \\
9-7-67 \\
9-21-67 \\
7-30-68 \\
9-17-68\end{array}$ & $\begin{array}{l}\mathrm{b} .02 \\
\mathrm{~b} .05 \\
1.04 \\
.05 \\
.04 \\
.07 \\
\mathrm{~b} .01 \\
.10 \\
.02\end{array}$ \\
\hline 31 & Neponset River & $\begin{array}{l}\text { Lat } 42^{\circ} 11^{\prime} 49^{\prime \prime}, \text { long } 71^{\circ} 09^{\prime} 19^{\prime \prime} \text {, } \\
\text { at Dedham St., } 3 \text { miles north } \\
\text { of Canton. }\end{array}$ & 82.1 & $\begin{array}{l}7-30-68 \\
9-17-68\end{array}$ & $\begin{array}{l}32.3 \\
32.0\end{array}$ \\
\hline 32 & $\begin{array}{l}\text { Pecun1t Brook } \\
\text { (01105552) }\end{array}$ & $\begin{array}{l}\text { Lat } 42^{\circ} \mathrm{MI}^{\prime} 21^{\prime \prime} \text {, long } 71^{\circ} 08^{\prime} 40^{\prime \prime} \text {, } \\
\text { at Elm St., } 2 \text { miles north of } \\
\text { Canton. }\end{array}$ & .79 & $\begin{array}{l}8-15-66 \\
9-13-66 \\
5-2-67 \\
8-18-67 \\
8-23-67 \\
9-7-67 \\
9-21-67 \\
7-30-68 \\
9-17-68\end{array}$ & $\begin{array}{l}0 \\
o \\
b .03 \\
b .006 \\
b .003 \\
b .013 \\
b .001 \\
b .05 \\
b .03\end{array}$ \\
\hline 33 & $\begin{array}{l}\text { Ponkapog Brook } \\
(01105554)\end{array}$ & $\begin{array}{l}\text { Lat } 42^{\circ} 12^{\prime} 12^{\prime \prime} \text {, long } 71^{\circ} 08^{\prime} 09^{\prime \prime} \text {, } \\
\text { at Elm St., } 3 \text { miles north of } \\
\text { Canton. }\end{array}$ & 3.78 & $\begin{array}{r}8-15-66 \\
9-13-66 \\
10-7-66 \\
5-2-67 \\
8-18-67 \\
8-22-67 \\
9-7-67 \\
9-21-67 \\
7-30-68 \\
9-17-68\end{array}$ & $\begin{array}{r}1.21 \\
.92 \\
2.32 \\
7.67 \\
2.44 \\
2.38 \\
2.16 \\
2.93 \\
4.29 \\
2.25\end{array}$ \\
\hline 34 & $\begin{array}{l}\text { Neponset River } \\
(01105556)\end{array}$ & $\begin{array}{l}\text { Lat } 42^{\circ} 12^{\prime} 33^{\prime \prime}, \text { long } 71^{\circ} 08^{\prime} 47^{\prime \prime} \text {, } \\
\text { at Greenlodge St., } 3 \text { miles } \\
\text { southeast of Dedham. }\end{array}$ & 88.5 & -- & (a) \\
\hline 35 & Neponset River & $\begin{array}{l}\text { Lat } 42^{\circ} 14^{\prime} 04^{\prime \prime}, \text { long } 71^{\circ} 07^{\prime} 23^{\prime \prime} \text {, } \\
\text { at Neponset Valley Parkway, } \\
3 \text { miles southwest of Milton. }\end{array}$ & 93.2 & $\begin{array}{l}7-30-68 \\
9-17-68\end{array}$ & $\begin{array}{l}35.2 \\
31.8\end{array}$ \\
\hline
\end{tabular}

WEYMOUIH FORE RIVER BASIN

\begin{tabular}{|c|c|c|c|c|c|}
\hline 36 & $\begin{array}{l}\text { Norroway Brook } \\
\text { (01105559) }\end{array}$ & $\begin{array}{l}\text { Lat } 42^{\circ} \mathrm{Jl} \mathrm{I}^{\prime} \mathrm{O} 4^{\prime \prime} \text {, long } 7 \mathrm{I}^{\circ} 03^{\prime} 08^{\prime \prime} \text {, } \\
\text { at dak St., I mile south of } \\
\text { North Randolph. }\end{array}$ & 2.57 & $\begin{array}{l}8-16-66 \\
9-12-66 \\
5-1-67 \\
8-17-67 \\
8-21-67 \\
9-6-67 \\
9-20-67 \\
9-20-68\end{array}$ & $\begin{array}{l}0 \\
0 \\
2.22 \\
.35 \\
.28 \\
.35 \\
.15 \\
.08\end{array}$ \\
\hline 37 & Farm Rlver & $\begin{array}{l}\text { Lat } 42^{\circ} 12^{\prime} 30^{\prime \prime} \text {, long } 71^{\circ} 02^{\prime} 27^{\prime \prime} \text {, } \\
\text { at West St., } 0.33 \mathrm{~m} 1 \mathrm{le} \text { below Great } \\
\text { Pond Reservolr outlet, } 2.25 \text { miles } \\
\text { southwest of Braintree. }\end{array}$ & 9.04 & $\begin{array}{l}8-16-66 \\
9-12-66 \\
5-1-67\end{array}$ & $\begin{array}{r}.09 \\
0.03 \\
15.9\end{array}$ \\
\hline 38 & $\begin{array}{l}\text { Farm River } \\
(01105562)\end{array}$ & $\begin{array}{l}\text { Lat } 42^{\circ} 11^{\prime} 55^{\prime \prime} \text {, long } 71^{\circ} 01^{\prime} 29^{\prime \prime} \text {, } \\
\text { at Pond St., i. } 25 \text { miles southwest } \\
\text { of South Braintree. }\end{array}$ & 10.1 & $\begin{array}{l}8-18-67 \\
8-21-67 \\
9-6-67 \\
9-20-67 \\
9-20-68\end{array}$ & $\begin{array}{r}2.30 \\
1.66 \\
2.34 \\
.87 \\
.84\end{array}$ \\
\hline
\end{tabular}


Table 4.--Stream sites and discharge measurements (Continued)

\begin{tabular}{|c|c|c|c|c|c|}
\hline $\begin{array}{l}\text { Map } \\
\text { number }\end{array}$ & $\begin{array}{c}\text { Station name and } \\
\text { U.S. Geological Survey } \\
\text { number }\end{array}$ & Location & $\begin{array}{l}\text { Dralnage } \\
\text { area } \\
\text { (sq mi })\end{array}$ & Date & $\begin{array}{l}\text { Discharge } \\
\text { (cublc feet } \\
\text { per second) }\end{array}$ \\
\hline \multicolumn{6}{|c|}{ WEYMOUTH FORE RIVER BASIN (ContInued) } \\
\hline 39 & Trout Brook & $\begin{array}{l}\text { Lat } 42^{\circ} 08^{\circ} 08^{\prime \prime} \text {, long } 71^{\circ} 00^{\prime} 44^{\prime \prime} \text {, } \\
0.5 \text { mile above inlet to Lake Holbrook, } \\
1.33 \text { miles south of Holbrook. }\end{array}$ & .95 & $\begin{array}{l}9-12-66 \\
5-2-67\end{array}$ & 2.14 \\
\hline 40 & $\begin{array}{l}\text { Trout Brook } \\
\text { (01105565) }\end{array}$ & $\begin{array}{l}\text { Lat } 42^{\circ} 08^{\prime} 25^{\prime \prime} \text {, long } 71^{\circ} 00^{\prime} 50^{\prime \prime} \text {, } \\
0.2 \text { mile above inlet to Lake Holbrook, } \\
\text { I mile south of Holbrook. }\end{array}$ & 1.12 & $\begin{array}{l}8-16-66 \\
8-21-67 \\
9-6-67 \\
9-20-67\end{array}$ & $\begin{array}{l}.03 \\
.23 \\
.28 \\
.04\end{array}$ \\
\hline 41 & $\begin{array}{l}\text { Cochato River tributary } \\
(01105567)\end{array}$ & $\begin{array}{l}\text { Lat } 42^{\circ} 08^{\prime 4} 40^{\prime \prime}, \text { long } 71^{\circ} 01^{\prime} 27^{\prime \prime} \text {, } \\
\text { at South St., I mile southwest of } \\
\text { Holbrook. }\end{array}$ & .89 & $\begin{array}{l}8-16-66 \\
9-12-66 \\
5-2-67 \\
8-17-67 \\
8-21-67 \\
9-6-67 \\
9-20-67\end{array}$ & $\begin{array}{l}0 \\
0 \\
1.95 \\
0 \\
0 \\
0 \\
0\end{array}$ \\
\hline 42 & Mary Lee Brook & $\begin{array}{l}\text { Lat } 42^{\circ} 09^{\prime} 39^{\prime \prime}, \text { long } 71^{\circ} 01^{\prime} 44^{\prime \prime} \\
\text { at Mill St., } 0.66 \mathrm{mile} \text { east of } \\
\text { Randolph. }\end{array}$ & 1.38 & $\begin{array}{l}5-1-67 \\
8-17-67 \\
8-21-67 \\
9-6-67 \\
9-20-67 \\
9-20-68\end{array}$ & $\begin{array}{r}3.51 \\
.62 \\
.46 \\
.34 \\
.12 \\
.18\end{array}$ \\
\hline 43 & $\begin{array}{l}\text { Glovers Brook } \\
\text { (01105571) }\end{array}$ & $\begin{array}{l}\text { Lat } 42^{\circ} 10^{\prime} 09^{\prime \prime}, \text { long } 71^{\circ} 02^{\prime} 12^{\prime \prime} \text {, } \\
\text { at North St., } 0.5 \text { mile northeast } \\
\text { of Randolph. }\end{array}$ & 2.50 & $\begin{array}{l}8-16-66 \\
9-12-66 \\
5-1-67 \\
8-17-67 \\
8-21-67 \\
9-6-67 \\
9-20-67 \\
9-20-68\end{array}$ & $\begin{array}{r}.22 \\
.20 \\
4.66 \\
.81 \\
.50 \\
.82 \\
.26 \\
.10\end{array}$ \\
\hline 44 & $\begin{array}{l}\text { Tumbling Brook } \\
\text { (01205573) }\end{array}$ & $\begin{array}{l}\text { Lat } 42^{\circ} 09^{\prime} 59^{\prime \prime}, \text { long } 71^{\circ} 01^{\prime} 13^{\prime \prime} \text {, } \\
\text { at Center St., I mile northwest } \\
\text { of Holbrook. }\end{array}$ & .93 & $\begin{array}{l}8-16-66 \\
5-2-67 \\
8-21-67 \\
9-6-67 \\
9-20-67\end{array}$ & $\begin{array}{l}0 \\
\text { b. } 04 \\
0 \\
0 \\
0\end{array}$ \\
\hline 45 & $\begin{array}{l}\text { Cochato River } \\
\text { (01105574) }\end{array}$ & $\begin{array}{l}\text { Lat } 42^{\circ} 10^{\prime 4} 49^{\prime \prime} \text {, long } 71^{\circ} 01^{\prime} 14^{\prime \prime} \text {, } \\
\text { at railway culvert, } 1,700 \text { feet } \\
\text { above Cranberry Brook, } 0.5 \text { mile } \\
\text { northwest of Bralntree Highlands. }\end{array}$ & 10.2 & -- & (a) \\
\hline 46 & $\begin{array}{l}\text { Cranberry Brook } \\
\text { (01105575) }\end{array}$ & $\begin{array}{l}\text { Lat } 42^{\circ} 11^{\prime} 02^{\prime \prime}, \text { long } 71^{\circ} 00^{\prime} 42^{\prime \prime} \text {, } \\
\text { at Washington St., } 0.5 \text { mile north } \\
\text { of Braintree Highlands. }\end{array}$ & 1.72 & $\begin{array}{r}8-16-66 \\
9-12-66 \\
10-7-66 \\
12-20-66 \\
1-26-67 \\
3-10-67 \\
5-2-67 \\
5-25-67 \\
7-12-67 \\
8-10-67 \\
8-17-67 \\
8-21-67 \\
9-6-67 \\
9-20-67 \\
10-11-67 \\
9-20-68\end{array}$ & $\begin{array}{r}.06 \\
.08 \\
.33 \\
1.96 \\
2.67 \\
8.68 \\
3.87 \\
23.2 \\
.98 \\
.84 \\
.16 \\
.27 \\
.21 \\
.06 \\
.23 \\
\mathrm{~b} .01\end{array}$ \\
\hline 47 & Sunset Lake Outlet & $\begin{array}{l}\text { Lat } 42^{\circ} 12^{\prime} 00^{\prime \prime} \text {, long } 72^{\circ} 00^{\prime} 56^{\prime \prime} \text {, } \\
\text { at Pond St., } 0.75 \text { mile southwest } \\
\text { of South Braintree. } \\
\end{array}$ & .51 & $\begin{array}{l}8-16-66 \\
9-12-66 \\
5-1-67 \\
8-18-67 \\
8-21-67 \\
9-6-67 \\
9-20-67\end{array}$ & $\begin{array}{l}.02 \\
.05 \\
.85 \\
.04 \\
.03 \\
.02 \\
.02\end{array}$ \\
\hline 48 & $\begin{array}{l}\text { Monatiquot River } \\
(01105580)\end{array}$ & $\begin{array}{l}\text { Lat } 42^{\circ} 11^{\prime} 51^{\prime \prime} \text {, long } 71^{\circ} 00^{\prime} 31^{\prime \prime} \text {, } \\
\text { at Jefferson St., South Braintree. }\end{array}$ & $24 \cdot 7$ & $\begin{array}{r}10-7-66 \\
12-20-66 \\
1-26-67 \\
3-10-67 \\
5-1-67 \\
5-25-67 \\
7-12-67 \\
8-10-67 \\
8-17-67 \\
8-21-67 \\
9-6-67 \\
9-20-67 \\
10-11-67\end{array}$ & $\begin{array}{c}6.08 \\
19.2 \\
37.5 \\
110 \\
53.5 \\
36.7 \\
19.7 \\
12.5 \\
7.94 \\
4.81 \\
7.22 \\
1.35 \\
7.73\end{array}$ \\
\hline
\end{tabular}


Table 4.--Stream sites and discharge measurements (Continued)

\begin{tabular}{|c|c|c|c|c|c|}
\hline $\begin{array}{l}\text { Map } \\
\text { number }\end{array}$ & $\begin{array}{c}\text { Station name and } \\
\text { U.S. Geological Survey } \\
\text { number }\end{array}$ & Location & $\begin{array}{l}\text { Drainage } \\
\text { area } \\
\text { (sq m1) }\end{array}$ & Date & $\begin{array}{l}\text { Discharge } \\
\text { (cubic feet } \\
\text { per second) }\end{array}$ \\
\hline \multicolumn{6}{|c|}{ WEYMOUTH FORE RIVER BASIN (Cont1nued) } \\
\hline \multirow[t]{2}{*}{49} & Honat1quot River & $\begin{array}{l}\text { Lat } 42^{\circ} 13^{\prime} 25^{\prime \prime}, \text { long } 70^{\circ} 59^{\prime} 49^{\prime \prime} \text {, } \\
\text { at Middle St., Braintree. }\end{array}$ & $27 \cdot 5$ & $\begin{array}{l}8-18-67 \\
8-21-67 \\
9-6-67 \\
9-20-67\end{array}$ & $\begin{array}{l}11.3 \\
7.36 \\
8.95 \\
4.26\end{array}$ \\
\hline & & WEYMOUTH BACK RIVER BASIN & & & \\
\hline 50 & Mill River & $\begin{array}{l}\text { Lat } 42^{\circ} 11^{\prime} 35^{\prime \prime}, \text { long } 70^{\circ} 57^{\prime} 35^{\prime \prime} \text {, } \\
\text { at Front St., } 1.33 \text { miles north } \\
\text { of South Weymouth. }\end{array}$ & 5.77 & $\begin{array}{r}8-16-66 \\
9-13-66 \\
10-7-66 \\
5-2-67 \\
8-18-67 \\
8-22-67 \\
9-7-67 \\
9-21-67\end{array}$ & $\begin{array}{r}.06 \\
.27 \\
.67 \\
9.67 \\
.84 \\
.49 \\
.38 \\
.15\end{array}$ \\
\hline 51 & Mill River & $\begin{array}{l}\text { Lat } 42^{\circ} 12^{\prime} 02^{\prime \prime}, \text { long } 70^{\circ} 56^{\prime} 48^{\prime \prime} \text {, } \\
\text { at Middle St., } 1.5 \text { miles south } \\
\text { of Weymouth. }\end{array}$ & 6.30 & $\begin{array}{l}9-13-66 \\
5-2-67 \\
8-18-67 \\
8-22-67 \\
9-7-67 \\
9-8-67 \\
9-21-67\end{array}$ & $\begin{array}{r}0 \\
10.4 \\
.68 \\
.25 \\
.20 \\
.11 \\
0\end{array}$ \\
\hline 52 & $\begin{array}{l}\text { Old Swamp River } \\
\text { (01105594) }\end{array}$ & $\begin{array}{l}\text { Lat } 42^{\circ} 08^{\prime} 59^{\prime \prime}, \text { long } 70^{\circ} 55^{\prime} 10^{\prime \prime} \text {, } \\
\text { at Forest St., Rockland. }\end{array}$ & .22 & $\begin{array}{r}7-12-67 \\
8-2-67 \\
8-18-67 \\
8-22-67 \\
9-7-67 \\
9-21-67 \\
10-16-67 \\
12-22-67 \\
7-31-68 \\
9-16-68 \\
9-24-68\end{array}$ & $\begin{array}{l}.27 \\
.23 \\
.12 \\
.10 \\
.08 \\
.02 \\
.17 \\
.41 \\
.07 \\
.02 \\
.01\end{array}$ \\
\hline 54 & Old Swamp River & $\begin{array}{l}\text { Lat } 42^{\circ} 10^{\prime} 40^{\prime \prime} \text {, long } 70^{\circ} 56^{\prime} 06^{\prime \prime} \text {, } \\
\text { at Ralph Talbot St., South Weymouth. }\end{array}$ & 3.24 & $\begin{array}{r}7-12-67 \\
8-2-67 \\
10-16-67 \\
12-22-67 \\
7-31-68 \\
9-16-68 \\
9-24-68\end{array}$ & $\begin{array}{r}2.28 \\
2.29 \\
1.14 \\
4.14 \\
.26 \\
.38 \\
.14\end{array}$ \\
\hline 55 & Old Swamp River & $\begin{array}{l}\text { Lat } 42^{\circ} 10^{\prime} 57^{\prime \prime} \text {, long } 70^{\circ} 56^{\prime} 08^{\prime \prime} \text {, } \\
\text { at Pine St., I mile northeast of } \\
\text { South Weymouth. }\end{array}$ & $c_{3.57}$ & $\begin{array}{l}7-28-59 \\
8-17-59 \\
9-15-59 \\
9-22-59 \\
7-13-60 \\
8-30-60\end{array}$ & $\begin{array}{l}3.26 \\
.63 \\
.65 \\
.45 \\
.36 \\
.28\end{array}$ \\
\hline 56 & $\begin{array}{l}\text { Old Swamp River } \\
\text { (01105598) }\end{array}$ & $\begin{array}{l}\text { Lat } 42^{\circ} 11^{\prime} 13^{\prime \prime} \text {, long } 70^{\circ} 56^{\prime} 02^{\prime \prime} \text {, } \\
\text { at Pleasant St., } 1.25 \text { miles } \\
\text { northeast of South Weynouth. }\end{array}$ & 3.65 & $\begin{array}{r}9-12-66 \\
10-7-66 \\
8-22-67 \\
9-7-67 \\
9-21-67\end{array}$ & $\begin{array}{r}2.41 \\
2.01 \\
1.01 \\
1.38 \\
.29\end{array}$ \\
\hline 57 & Old Swamp River & $\begin{array}{l}\text { Lat } 42^{\circ} 11^{\prime} 25^{\prime \prime}, \text { long } 70^{\circ} 56^{\prime 4} 43^{\prime \prime} \text {, } \\
\text { between divided lanes of State } \\
\text { Highway } 3 \text { and } 128,1.2 \text { miles } \\
\text { north of South Weymouth. Gaging } \\
\text { station data available since } \\
\text { May } 1966 \text { (see U.S. Geological } \\
\text { Survey, 1966-73). }\end{array}$ & 4.29 & & \\
\hline 58 & $\begin{array}{l}\text { Whitmans Pond Outlet } \\
\text { (head of Weymouth } \\
\text { Back River) }\end{array}$ & $\begin{array}{l}\text { Lat } 42^{\circ} 12^{\prime} / 45^{\prime \prime}, \text { long } 70^{\circ} 55^{\prime} 32^{\prime \prime} \text {, } \\
\text { at Pleasant St., East Weymouth. }\end{array}$ & $12 \cdot 3$ & $\begin{array}{r}9-13-66 \\
10-10-66 \\
5-2-67 \\
8-18-67 \\
8-22-67 \\
9-7-67 \\
9-20-67\end{array}$ & $\begin{array}{r}2.60 \\
1.79 \\
21.1 \\
1.23 \\
1.13 \\
3.75 \\
.49\end{array}$ \\
\hline
\end{tabular}


Table 4.--Stream sites and discharge measurements (Continued)

\begin{tabular}{|c|c|c|c|c|c|}
\hline $\begin{array}{c}\text { Map } \\
\text { number }\end{array}$ & $\begin{array}{c}\text { Station name and } \\
\text { U.S. Geological Survey } \\
\text { number }\end{array}$ & Location & $\begin{array}{l}\text { Drainage } \\
\text { area } \\
(\mathrm{sq} \mathrm{ml})\end{array}$ & Date & $\begin{array}{l}\text { Discharge } \\
\text { (cublc feet } \\
\text { per second) }\end{array}$ \\
\hline 59 & $\begin{array}{l}\text { Whitmans Pond Outlet } \\
\text { tributary }\end{array}$ & $\begin{array}{l}\text { Lat } 42^{\circ} 12^{\prime} 45^{\prime \prime} \text {, long } 70^{\circ} 55^{\prime} 30^{\prime \prime} \text {, } \\
\text { at Water St., East Weymouth. }\end{array}$ & 0.56 & $9-13-66$ & 0 \\
\hline 60 & $\begin{array}{l}\text { Whitmans Pond Outlet } \\
\text { tributary No. } 2 \\
(01105614)\end{array}$ & $\begin{array}{l}\text { Lat } 42^{\circ} 12^{\prime} 52^{\prime \prime} \text {, Iong } 70^{\circ} 55^{\prime} 08^{\prime \prime} \text {, } \\
\text { at Broad St., East Weymouth. }\end{array}$ & .38 & $\begin{array}{l}9-13-66 \\
5-2-67 \\
8-18-67 \\
8-22-67 \\
9-23-68\end{array}$ & $\begin{array}{l}0 \\
.89 \\
.04 \\
.009\end{array}$ \\
\hline 61 & $\begin{array}{l}\text { Fresh River } \\
(01105617)\end{array}$ & $\begin{array}{l}\text { Lat } 42^{\circ} 13^{\prime} 30^{\prime \prime} \text {, long } 70^{\circ} 54^{\prime} 53^{\prime \prime} \text {, } \\
\text { at Comnercial St., } 1.75 \mathrm{miles} \\
\text { southwest of Hingham. }\end{array}$ & .91 & $\begin{array}{r}9-13-66 \\
10-10-66 \\
5-2-67 \\
8-18-67 \\
8-22-67 \\
9-7-67 \\
9-20-67 \\
9-23-68\end{array}$ & $\begin{array}{r}.54 \\
.54 \\
2.09 \\
.56 \\
.67 \\
.60 \\
.40 \\
.17\end{array}$ \\
\hline
\end{tabular}

a Chemical data only available (see table 5).

b Field estimate of discharge.

c Previously published as 3.70 square miles. 
TABLE 5.--CHEMICAL ANALYSES OF SURFACE WATER

\begin{tabular}{|c|c|c|c|c|c|c|c|c|c|c|c|c|}
\hline ITE & $\begin{array}{l}\text { OIS- } \\
\text { CHARGE } \\
\text { (CFS) }\end{array}$ & $\begin{array}{l}\text { OIS- } \\
\text { SOLVED } \\
\text { SILICA } \\
\text { (SIN2) } \\
(\mathrm{MG} / \mathrm{L})\end{array}$ & $\begin{array}{l}\text { OIS- } \\
\text { SOLVEO } \\
\text { IROH } \\
\text { (FE) } \\
(U G / L)\end{array}$ & $\begin{array}{l}\text { OIS- } \\
\text { SOLVED } \\
\text { MAN- } \\
\text { GANESE } \\
\text { (MN) } \\
\text { (UG/L) }\end{array}$ & $\begin{array}{l}\text { OIS- } \\
\text { SOLVED } \\
\text { CAL- } \\
\text { CIUM } \\
\text { (CA) } \\
\text { (MG/L) }\end{array}$ & $\begin{array}{l}\text { DIS- } \\
\text { SOLVED } \\
\text { MAGNE- } \\
\text { SIUY } \\
(\text { YG) } \\
\text { (MG/L) }\end{array}$ & $\begin{array}{l}\text { DIS- } \\
\text { SOLVED } \\
\text { SCOIUM } \\
\text { (NA) } \\
\text { (YG/L) }\end{array}$ & $\begin{array}{l}\text { DIS- } \\
\text { SOLVED } \\
\text { POTAS- } \\
\text { SIUM } \\
\text { (K) } \\
\text { (MC/L) }\end{array}$ & $\begin{array}{l}\text { BICAR- } \\
\text { BONATE } \\
\text { (HCO3) } \\
\text { (MG/L) }\end{array}$ & $\begin{array}{l}\text { DIS- } \\
\text { SOLVED } \\
\text { SULFATE } \\
\text { (SO4) } \\
\text { (MG/L) }\end{array}$ & $\begin{array}{l}\text { DIS- } \\
\text { SOLVED } \\
\text { CHLO- } \\
\text { RIDE } \\
(C L) \\
\text { (MG/L) }\end{array}$ & $\begin{array}{l}\text { DIS- } \\
\text { SOLVED } \\
\text { FLUD- } \\
\text { RIDE } \\
\text { (F) } \\
\text { (HG/L) }\end{array}$ \\
\hline
\end{tabular}

011048 CO - NEPJNSET RIVER NEAR FOXAJRO MASS (LAT 420508 LONG 071 15 25)

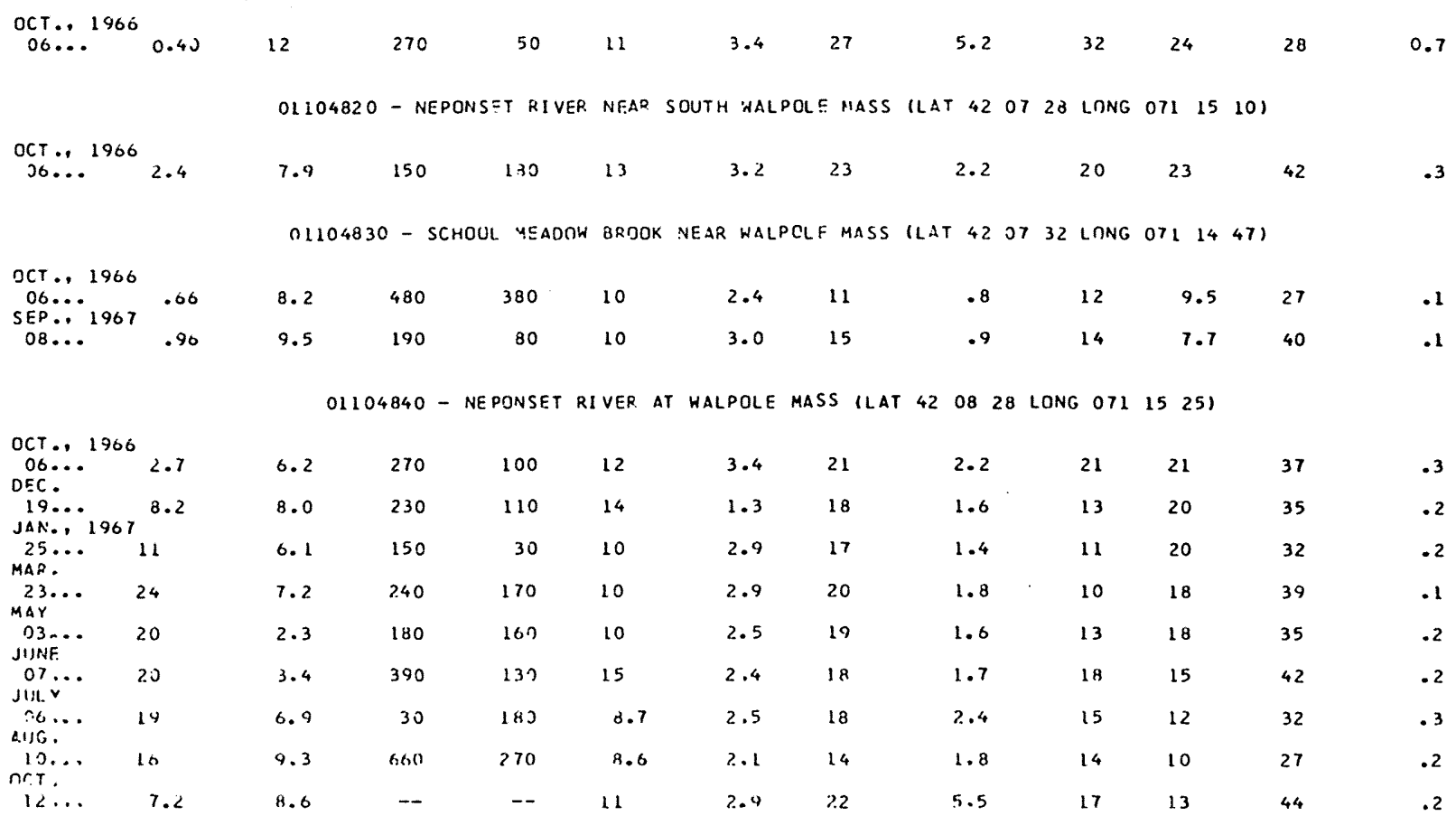

01104850 - MINE BROCK AT WALPOLE MASS (LAT 420914 LONG 0711552 )

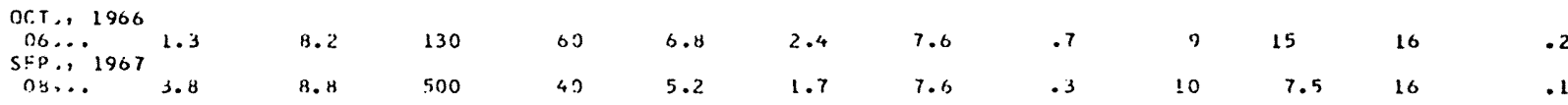

01104860 - SPRING BROOK AT WALPOI.E MASS (1.AT 42 O8 47 LONG O71 14 59)

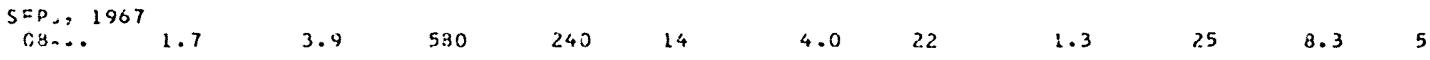

O110489C - NEPONST T RIVER AT EAST WALPOLE MASS (LAT 420947 LNNG $071 \quad 1256$ )

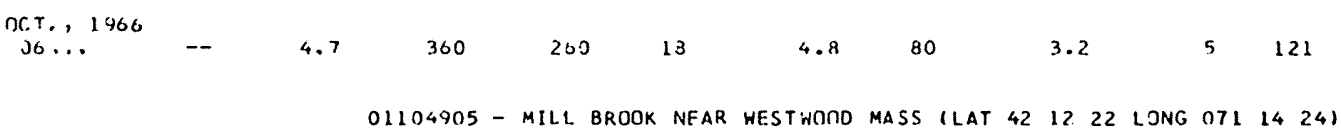


TABLE 5.--CHEMICAL ANALYSES OF SURFACE WATER

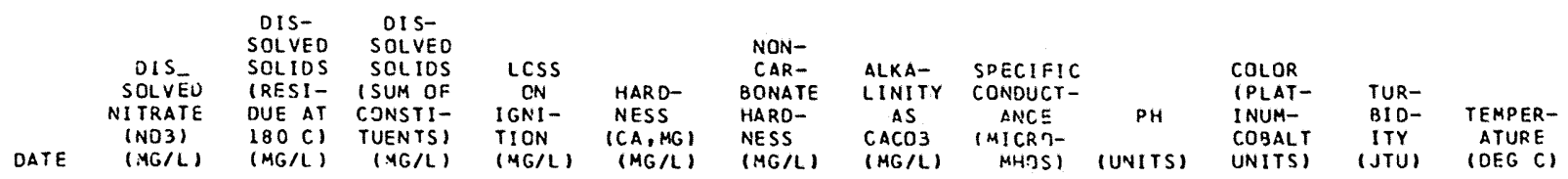

01104300 - NEPONSFT RIVER NEAR FOXAORO MASS (LAT 420508 LONG 071 15 25)

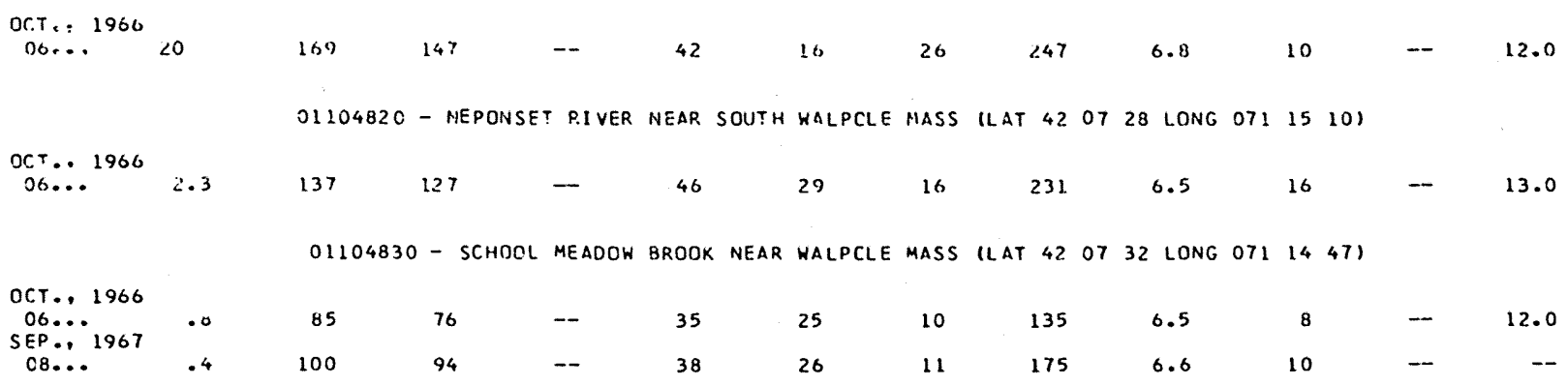

01104840 - NEPONSET RIVER AT WALPOLE MASS (LAT 420828 LONG 0711525 )

\begin{tabular}{|c|c|c|c|c|c|c|c|c|c|c|c|c|}
\hline $\begin{array}{l}\text { OCT.. } 1966 \\
\text { O6... } \\
\text { OEC. }\end{array}$ & .6 & 134 & 114 & -- & 44 & 27 & 17 & 214 & 6.7 & 15 & - & 13.0 \\
\hline $\begin{array}{l}19 \ldots 1967 \\
\text { JAN.. } 196\end{array}$ & 2.2 & 120 & 107 & 10 & 40 & 30 & 11 & 190 & 6.3 & 51 & -- & 3.0 \\
\hline $\begin{array}{l}25 \ldots \\
\text { MAR. }\end{array}$ & 2.4 & 111 & 97 & 19 & 37 & 28 & 9 & 178 & 6.3 & 20 & - & 4.0 \\
\hline $23 \ldots$ & 2.1 & 114 & 106 & 13 & 37 & 29 & 8 & 197 & 64.0 & 25 & 4 & 1.0 \\
\hline JUNF & .9 & 113 & 96 & 17 & 35 & 24 & 11 & 184 & 6.8 & 50 & 2 & 13.0 \\
\hline JuL $\ddot{Y}$ & 1.7 & 111 & 108 & 10 & 48 & 32 & 15 & 175 & 6.5 & 50 & 3 & 19.5 \\
\hline $\begin{array}{l}\text { C6... } \\
\text { AUG. }\end{array}$ & 3.0 & 110 & 94 & 22 & 32 & 20 & 12 & 169 & 6.4 & 150 & 3 & 19.0 \\
\hline $\begin{array}{c}10 \ldots \\
\text { gr.t. }\end{array}$ & 2.3 & 116 & 82 & 33 & 30 & 18 & 11 & 146 & 0.4 & 180 & -- & 23.0 \\
\hline $12 \ldots$ & .6 & 143 & 116 & -- & 40 & 26 & 14 & 213 & 5.3 & 55 & -- & 14.0 \\
\hline
\end{tabular}

01164850 - MINE BROOK AT WALPOLF MASS ILAT 423914 LONG 07115521

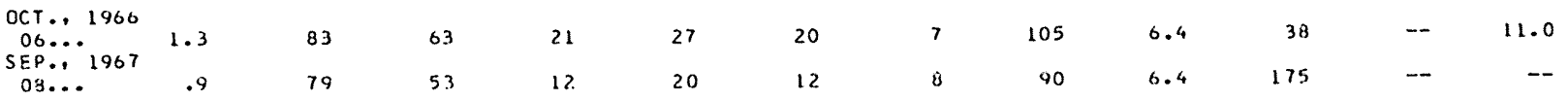

01104360 - SPRING 8ROOK AT WALPCLF MASS ILAT 42 OR 47 LONG 07114591

SFP., 1967

$08 \ldots .129$

117

01104890 - NEPONSFT RIVER AT EAST WALPOLF MASS (LAT 47.0947 LOHG 071 12. 56 )

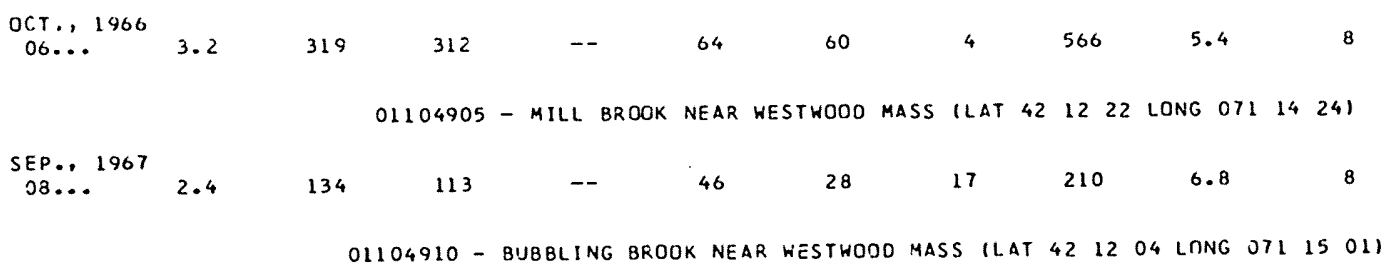

SEP., 1967

$08 \ldots$

$97 \quad 93 \quad--$

01104940 - WILLETT POND DUTLET NEAR NORHCOO MASS (LAT 421050 LNNG $071 \quad 14001$

SEP.. 1966

79

$74 \quad--\quad 30$

18

12

141

6.6

10

$-$

15.5 
TABLE 5.--CHEMICAL ANALYSES OF SURFACE WATER (CONTINUED)

\begin{tabular}{|c|c|c|c|c|c|c|c|c|c|c|c|c|}
\hline & $\begin{array}{l}\text { DIS- } \\
\text { CHARGE } \\
\text { (CFS) }\end{array}$ & $\begin{array}{l}\text { DIS- } \\
\text { SOLVED } \\
\text { SILICA } \\
\text { (SIOL) } \\
\text { (MG/L) }\end{array}$ & $\begin{array}{l}\text { OIS- } \\
\text { SOLVEO } \\
\text { IRCN } \\
\text { (FS) } \\
\text { (UG/L) }\end{array}$ & $\begin{array}{l}\text { DIS- } \\
\text { SOLVED } \\
\text { YAN- } \\
\text { GAAESE } \\
\text { (MN) } \\
\text { (UG/L) }\end{array}$ & $\begin{array}{l}\text { DIS- } \\
\text { SOLVED } \\
\text { CAL- } \\
\text { CIUM } \\
\text { (CA) } \\
\text { (MG/L) }\end{array}$ & $\begin{array}{l}\text { DIS- } \\
\text { SOLVFE } \\
\text { MAGNE- } \\
\text { SIUM } \\
(M G) \\
(M G / L)\end{array}$ & $\begin{array}{l}\text { DIS- } \\
\text { SOLVED } \\
\text { SODIUM } \\
\text { (NA) } \\
\text { (MG/L) }\end{array}$ & $\begin{array}{l}\text { DIS- } \\
\text { SOLVEO } \\
\text { POTAS- } \\
\text { SIUM } \\
\text { (K) } \\
\text { (MG/L) }\end{array}$ & $\begin{array}{l}\text { BICAR- } \\
\text { BONATE } \\
\text { (HCO3) } \\
\text { (HG/I.) }\end{array}$ & $\begin{array}{l}\text { DIS- } \\
\text { SOLVED } \\
\text { SULFATE } \\
\text { (SO4) } \\
\text { (MG/L) }\end{array}$ & $\begin{array}{l}\text { DIS- } \\
\text { SOLVEO } \\
\text { CHLO- } \\
\text { RIDE } \\
\text { (.CL) } \\
\text { (MG/L) }\end{array}$ & $\begin{array}{l}\text { DIS- } \\
\text { SOL VED } \\
\text { FLUO- } \\
\text { RIDE } \\
\text { (F) } \\
\text { (MG/L) }\end{array}$ \\
\hline
\end{tabular}

01104960 - GERMANY BROOK NEAR NORWOOD MASS (LAT 421104 LONG 0711329 )

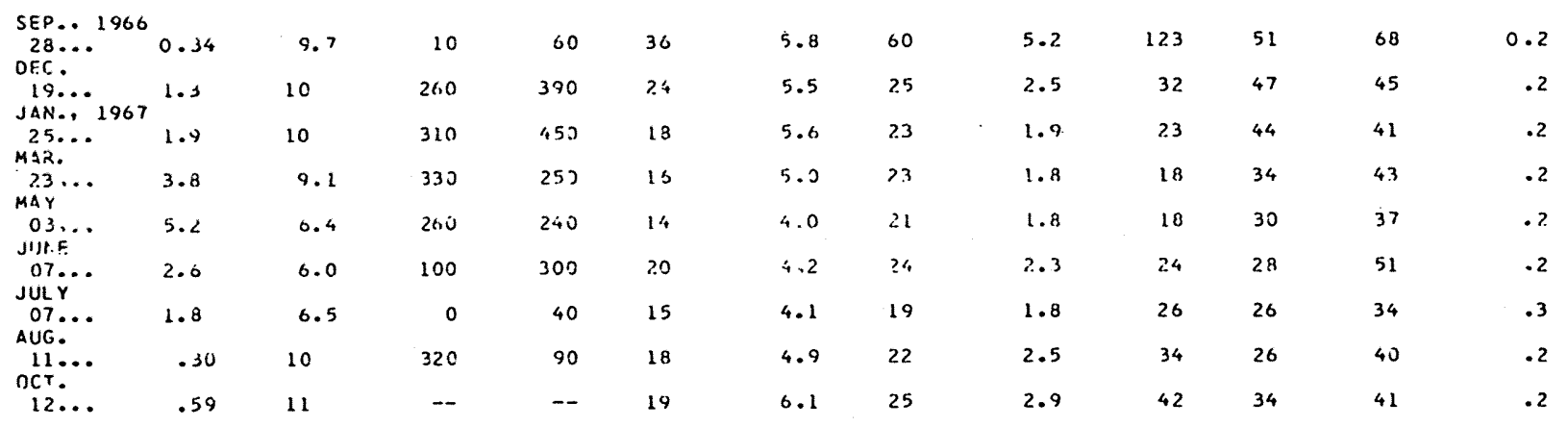

01104980 - HAWES BROOK AT NORWDOD MASS (LAT 421026 LONG 0711231 )

\begin{tabular}{|c|c|c|c|c|c|c|c|c|c|c|c|c|}
\hline $\begin{array}{l}\text { OB.... } \\
\text { DF.C. }\end{array}$ & .74 & 1.9 & 40 & 50 & 12 & 3.1 & 18 & 1.7 & 27 & 12 & 32 & .2 \\
\hline $\begin{array}{l}19 \ldots \ldots 1967 \\
\text { JAN.. } 196\end{array}$ & 2.7 & 4.7 & 140 & 120 & 20 & 3.4 & 25 & 2.0 & 28 & 32 & 47 & .2 \\
\hline $\begin{array}{l}25 \ldots \\
\text { MAR. }\end{array}$ & 4.0 & 5.6 & 140 & 210 & 17 & 4.5 & 26 & 1.9 & 24 & 34 & 46 & .1 \\
\hline$\underset{\text { MAY }}{23} \cdots$ & 22 & 4.9 & 60 & 100 & 12 & 3.3 & 46 & 1.6 & 15 & 23 & 77 & .2 \\
\hline $\begin{array}{l}03 . . . \\
\text { JURF. }\end{array}$ & 10 & 3.4 & 100 & 130 & 12 & 3.0 & 19 & 1.5 & 16 & 24 & 34 & .2 \\
\hline $\begin{array}{l}07 . \cdots \\
\text { JIL }\end{array}$ & 12 & 2.9 & 290 & 370 & 12 & 3.0 & 20 & 2.0 & 38 & 15 & 29 & .3 \\
\hline $\begin{array}{l}.77 \ldots \\
\text { Ails . }\end{array}$ & 12 & 6.2 & 10 & $7 n$ & 10 & 2.9 & 16 & 1.6 & 19 & 24 & 24 & .2 \\
\hline nc. & 0.4 & 2.1 & 310 & 60 & 14 & 2.0 & 16 & 1.8 & 2.4 & 71 & 29 & .1 \\
\hline $12 \ldots$ & 2.8 & 2.5 & $-\infty$ & -- & 12 & 3.3 & 17 & 1.4 & 26. & 14 & 32 & 2 \\
\hline
\end{tabular}

01105000 - NEPONSET RIVEF AT NORWDDO MASS. (LAT 421039 LDNE 071 12 0501 )

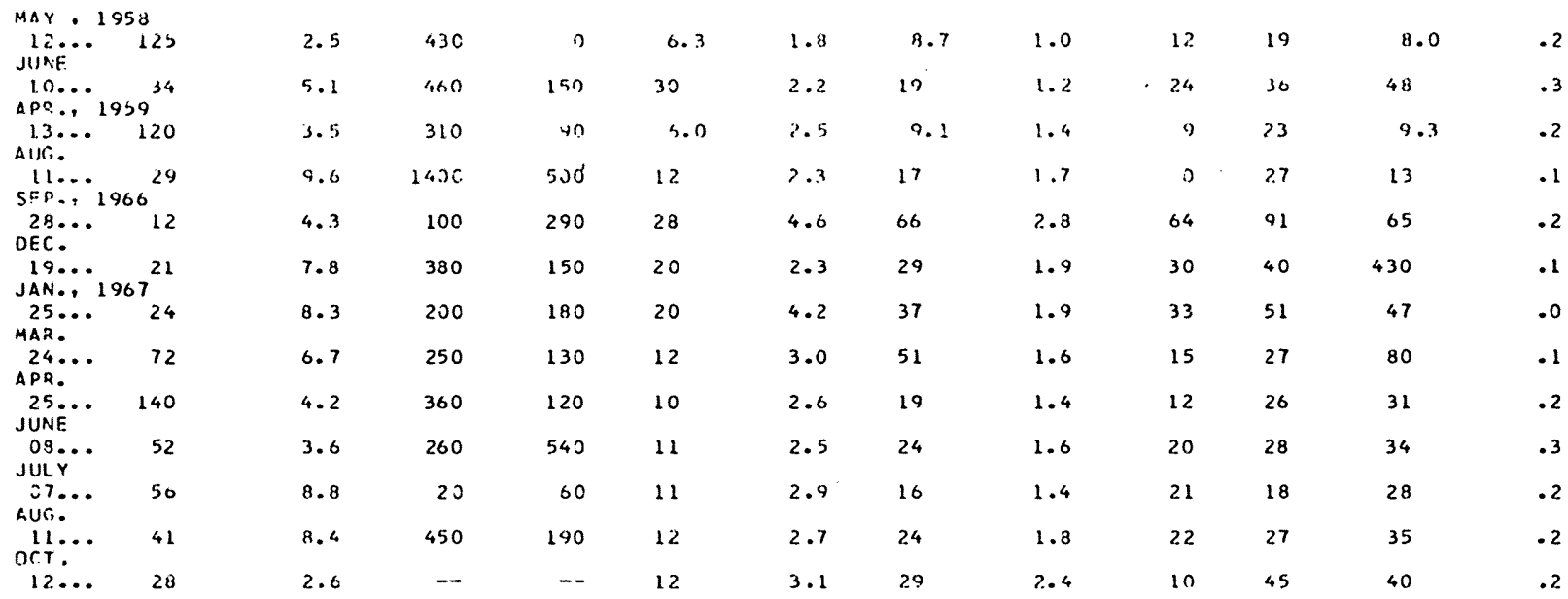

O1105100 - TRAPHCLE BROIK NEAR NOFHUOO MASS (LAT 42 O9 36 LONG $071 \quad 1147$ )

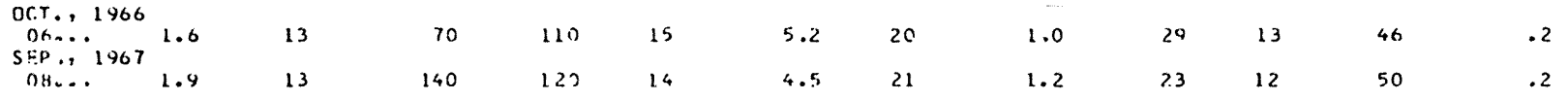


TABLE 5.--CHEMICAL ANALYSES OF SURFACE WATER (CONTINUED)

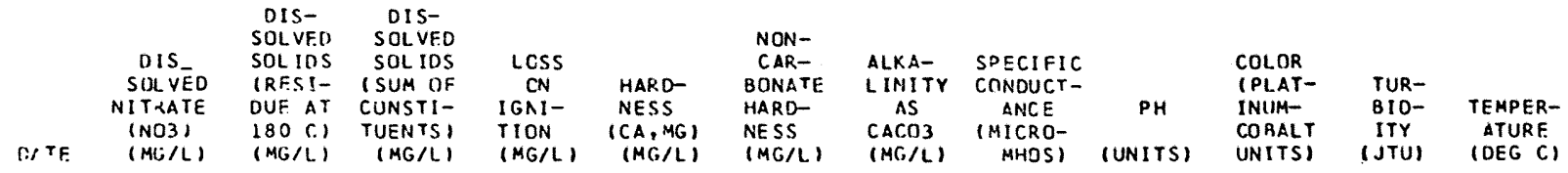

01104960 - GERMANY BROIIK NEAR NORWDOD MASS (LAT 421104 LONG 0711329 )

\begin{tabular}{|c|c|c|c|c|c|c|c|c|c|c|c|c|}
\hline $\begin{array}{l}211 \ldots \\
D \leq C .\end{array}$ & 1.2 & 290 & 297 & -- & 114 & 13 & 101 & 515 & 7.5 & 8 & - & 14.0 \\
\hline $\begin{array}{l}19 \ldots . .1967 \\
\text { JAN., } 1967\end{array}$ & 2.5 & 201 & 178 & 23 & 82 & 56 & 26 & 303 & 6.7 & 59 & -- & 3.5 \\
\hline $\begin{array}{r}25 \ldots \\
\text { MAR... }\end{array}$ & 2.4 & 169 & 157 & 20 & 68 & 49 & 19 & 273 & 6.5 & 55 & - & 6.0 \\
\hline MAY $23 \ldots$ & 3.2 & 169 & 144 & 36 & 60 & 46 & 15 & 260 & 6.6 & 50 & 3 & 3.5 \\
\hline JUNE & 1.9 & 137 & 125 & 16 & 52 & 36 & 15 & 225 & 7.1 & 55 & 6 & 13.0 \\
\hline $\begin{array}{l}07 \ldots \\
\text { AUG... }\end{array}$ & 2.9 & 142 & 123 & 26 & 54 & 33 & 21 & 218 & 7.0 & 148 & 4 & 15.5 \\
\hline OCT... & 2.4 & 167 & 143 & 25 & 65 & 37 & 28 & 247 & 7.1 & 120 & - & 16.5 \\
\hline $12 \ldots$ & 2.1 & 200 & 162 & -- & 72 & 38 & 34 & 292 & 7.3 & 80 & - & 11.0 \\
\hline
\end{tabular}

01104980 - HAWES BROOK AT NORWOOD MASS (LAT 421026 LONG OT1 1231 )

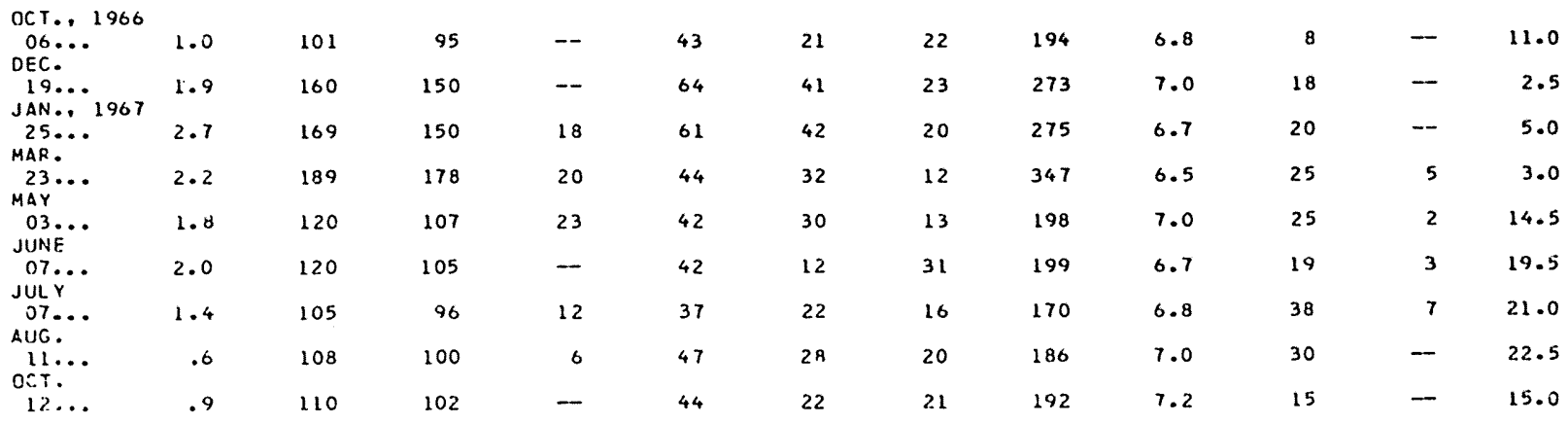

01105000 - INEPONSET RI VER AT NORWOOO NASS. (LAT 421039 LONG $071 \quad 120501$ )

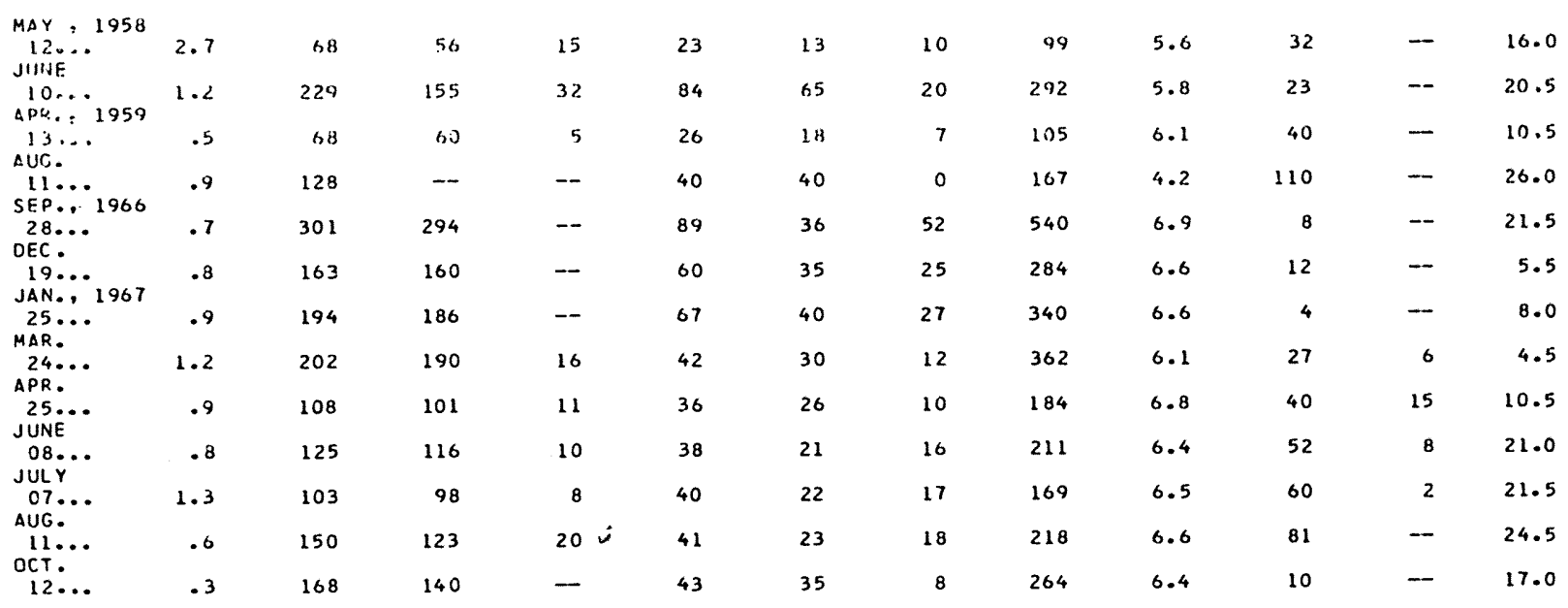

01105100 - TRAPHOLE BROOK NEAR NORWDOD MASS (LAT 420936 LONG 0711147 )

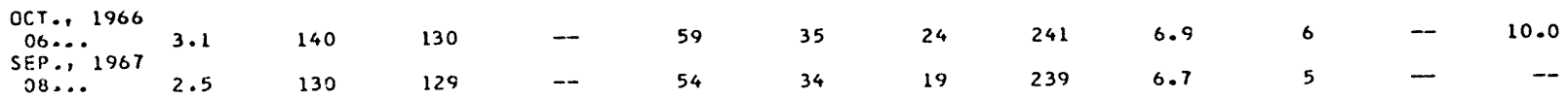


TABLE 5.--CHEMICAL ANALYSES OF SURFACE WATER (CONTINUED)

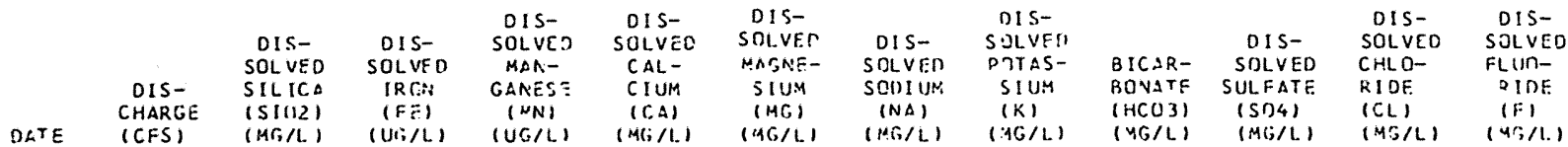

O1105200 - MASSGPOAG BQOOK AT SHAZON MASS (IAT 4L O6 53 LOVG O71 1019 )

\begin{tabular}{|c|c|c|c|c|c|c|c|c|c|c|c|}
\hline $\begin{array}{l}06 \ldots 1 \\
550.1167\end{array}$ & 1.7 & 1.1 & 30 & 150 & 4.3 & 1.5 & 6.3 & 0.9 & 3 & 16 & 10 \\
\hline $33 \ldots$ & 2.0 & 3.6 & 320 & (3) & 4.7 & 1.6 & 7.7 & .7 & 10 & 11 & 14 \\
\hline
\end{tabular}

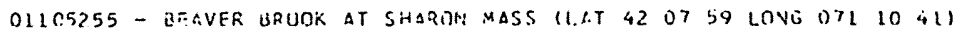

STP.: 1967

(iy... 1967 1.4

3.) $11 \quad 4.14$

.311

25

.2

OL10530C - STEPD HILL RFONK AT CANTCN MASS (LAT 42 O\& 39 LOAE O7L JB 14)

B.T = 1906

S:? 1467

8.3 14\% $\because 50$ in $3.1 \quad 14$

)

$\begin{array}{llllll}6.6 & 2.0 & 11.1 & 3.6 & 3.0 & 14\end{array}$

$1.13 \quad 2.2 \quad 17$

26

$\cdot 2$

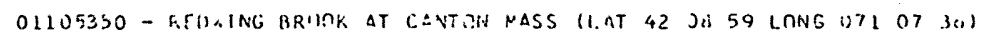

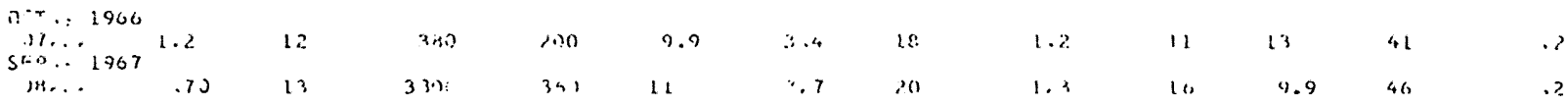

01105450 - DENIJIO BQOSK AT CANTMH MASS (LLT 421003 LONG OT1 J8 04)

OCT 1960

SEP. . $1967^{1.6}$

$\begin{array}{llllll}.4 & 80 & 40 & 10 & 3.8 & 19\end{array}$

$1.4 \quad 8 \quad 22$

38

$08 \ldots 2.2$

$3.4 \quad 1500$

$\begin{array}{llll}170 & 8.2 & 2.8 & 20\end{array}$

2.0

1

15

37

$\cdot 2$

01105500 - EAST BRANCH NEPONSET RIVER AT CANTON MASS (LAT 420916 LONG 0710847 )

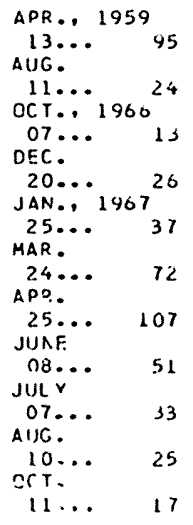

$2.5 \quad 320$

$8.4 \quad 560$

40

5.52 .0

6.7

1.3811

11

$5.5 \quad 120$

8.02 .2

7.6

1.2

$20 \quad 10$

12

190

$9.9 \quad 3.0 \quad 14$

1.2

$17 \quad 17$

$1.5 \quad 11 \quad 20$

$\begin{array}{llllll}6.4 & 140 & 110 & 14 & 1.4 & 16 \\ 6.1 & 120 & 130 & 9.2 & 2.7 & 15\end{array}$

$1.3 \quad 11$

$1.4 \quad 10$

$\begin{array}{llllll}6.4 & 100 & 70 & 9.0 & 2.7 & 22\end{array}$

$\begin{array}{llllll}3.0 & 100 & 80 & 8.5 & 2.3 & 15\end{array}$

$1.2 \quad 12$

$1.5 \quad 18$

1.22

1.223

1,7

20

O1105530 - PURGATORY BRUOK NEAR NIRWOOD MASS (LAT 4? 1233 LONG 071 11 06)

SFP, 1966

SFP. 21967

SFP. 1967

9.1

$100 \quad 210 \quad 46$

$3.8 \quad 147$

3. 6

50

$7.0 \quad 110$

3.5

$36 \quad 33$

272

01105554 - PONKAPOG BRUOK NEAR CANTON MASS (LAT 421.212 LONG 071 OB 09 )

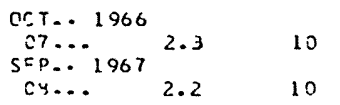

$\begin{array}{rrrrrrrrrr}260 & 60 & 14 & 4.5 & 20 & 1.5 & 23 & 18 & 39 & .2 \\ 600 & 140 & 15 & 4.5 & 22 & 1.6 & 22 & 14 & 49 & .2\end{array}$

01105556 - NEPONSET RIVER NEAR DEDHAM MASS (LAT $4212 \quad 33$ LONG 0710847 )

OCT., 1960

$07 . .$.

5.9

730

$20 \quad 15$

$3.7 \quad 23$

2.0

$40 \quad 24$

34

.2 
TABLE 5.--CHEMICAL ANALYSES OF SURFACE WATER (CONTINUED)

\begin{tabular}{|c|c|c|c|c|c|c|c|c|c|c|c|}
\hline $\begin{array}{l}\text { DIS } \\
\text { SOLVEO } \\
\text { NITRATE } \\
\text { (NO3) } \\
\text { (MG/L) }\end{array}$ & $\begin{array}{l}\text { OIS- } \\
\text { SOLVED } \\
\text { SOLIDS } \\
\text { IRESI- } \\
\text { DUE AT } \\
\text { I80C) } \\
\text { (MG/L) }\end{array}$ & $\begin{array}{l}\text { OIS- } \\
\text { SOL VED } \\
\text { SOLIDS } \\
\text { ISUM OF } \\
\text { CONSTI- } \\
\text { TUENTS) } \\
\text { (MG/L) }\end{array}$ & $\begin{array}{l}\text { LCSS } \\
\text { CN } \\
\text { IGNI- } \\
\text { TION } \\
(\mathrm{MG} / \mathrm{L})\end{array}$ & $\begin{array}{l}\text { HARO- } \\
\text { NESS } \\
\text { (CA,MG) } \\
\text { (MG/L) }\end{array}$ & $\begin{array}{l}\text { NON- } \\
\text { CAR- } \\
\text { BONATE } \\
\text { HARD- } \\
\text { NESS } \\
\text { (MG/L) }\end{array}$ & $\begin{array}{l}\text { ALKA- } \\
\text { LINITY } \\
\text { AS } \\
\text { CACO3 } \\
\text { (MG/L) }\end{array}$ & $\begin{array}{l}\text { SPECIFIC } \\
\text { CONOUCT- } \\
\text { ANCE } \\
\text { (MICRO- } \\
\text { MHOS) }\end{array}$ & $\begin{array}{c}\text { PH } \\
\text { (UNITS) }\end{array}$ & $\begin{array}{l}\text { COLOR } \\
\text { (PLAT- } \\
\text { INUM- } \\
\text { COBALT } \\
\text { (UNITS) }\end{array}$ & $\begin{array}{l}\text { TUR- } \\
\text { BID- } \\
\text { ITY } \\
\text { (JTU) }\end{array}$ & $\begin{array}{l}\text { TEMPER- } \\
\text { ATURE } \\
\text { (DEG C) }\end{array}$ \\
\hline
\end{tabular}

01105200 - MASSAPOAG BROOK AT SHARON MASS (LAT 420653 LONG $071 \quad 1019$ )

\begin{tabular}{|c|c|c|c|c|c|c|c|c|c|c|c|c|}
\hline $\begin{array}{l}\text { O6.... } \\
\text { SEP.., } 1967\end{array}$ & 3.3 & 49 & 46 & - & 16 & 14 & 2 & 86 & 5.5 & 3 & - & 14.5 \\
\hline $08 \ldots$ & .2 & 60 & 49 & -- & 18 & 10 & 8 & 91 & 6.4 & 10 & -- & - \\
\hline
\end{tabular}

01105255 - EEAVER BROOK AT SHARON MASS ILAT 42 OY 59 LONG 0711041 )

SEP., 1967
$08 . .$.

1.5

111

01105300 - STEEP HILL BROOK AT CANTON MASS (LAT 42 O8 39 LONG OT1 08 14)

OCT., 1966

SEP....

$$
98
$$

104

OCT.: 1966

C7...

SEP $1967 \quad 2.0 \quad 131$

173

106

115

31

38

30

30

$\begin{array}{rr}9 & 134 \\ 13 & 206\end{array}$

6.565

$-\quad 10.5$

01105450 - PEQUID BROOK AT CANTON MASS (I.AT 421003 LONG OT1 OB 04 )

\begin{tabular}{|c|c|c|c|c|c|c|c|c|c|c|c|c|}
\hline $\begin{array}{l}07 \ldots . . \\
\text { SโP.: } 1907\end{array}$ & 5.6 & 153 & 104 & 47 & 40 & 34 & 7 & 201 & 6.4 & 24 & -- & 13.5 \\
\hline $04 . .$. & 1.2 & 113 & 47 & 17 & 32 & 20 & 11 & 194 & 6.2 & 100 & -- & $-\infty$ \\
\hline
\end{tabular}

\begin{tabular}{|c|c|c|c|c|}
\hline $\begin{array}{l}\text { APR.. } 1959 \\
13 \ldots \ldots \\
\text { AUG. }\end{array}$ & 1.3 & 58 & 45 & 9 \\
\hline 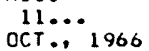 & 1.4 & 76 & 61 & 8 \\
\hline $\begin{array}{l}07 \ldots . . . \\
\text { DEC.. }\end{array}$ & 2.1 & 96 & 89 & -- \\
\hline $\begin{array}{l}20 \ldots . .1967 \\
\text { JAN., } 1967\end{array}$ & 1.6 & 110 & 100 & 7 \\
\hline $\begin{array}{l}25 \ldots . . \\
\text { MAR. }\end{array}$ & 2.0 & 96 & 89 & -- \\
\hline $\begin{array}{l}24 \ldots \\
A P R\end{array}$ & 1.9 & 113 & 106 & 9 \\
\hline $\begin{array}{l}25 \ldots . . \\
\text { JUNF. }\end{array}$ & $1 \cdot 3$ & 94 & 82 & 13 \\
\hline JUL 08. & 2.3 & 110 & 88 & 20 \\
\hline $\begin{array}{l}\text { C7... } \\
\text { AUG. }\end{array}$ & 1.2 & 92 & 83 & 10 \\
\hline $\begin{array}{l}10 . \\
O C T .\end{array}$ & .9 & 95 & 82 & 12 \\
\hline $11 \ldots$ & .9 & 111 & 88 & -- \\
\hline
\end{tabular}

01105530 - PURGATIRY BROOK NEAR NORWOOC MASS (LAT 421233 LING 071 11 06 )

SFP.: 1966
$28 \ldots$
SF. . 1967
OB. 196 68

521

$\begin{array}{lll}558 & -- & 151 \\ 434 & -- & 122\end{array}$

110

41

1050

7.1

5.922

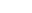

10.5

$\begin{array}{llll}9 & 13 & 16 & 98\end{array}$

$5.7 \quad 80$

$6.7 \quad 14$

$6.5 \quad 29$

6.4

6.5

6.9

6.3

6.7

7.0

40

$--\quad 24.5$

- 11.5

- 2.5

- 4.0

- 2.5

39.5

319.0

222.0

$-\quad 23.5$

0110555\% - PCNKAPCG BROOK NEAR CANTON MASS (LAT 421212 LONG 0710809 )

NC.T. 1960

27... 1960

$165 \quad 120 \quad 32$

151

13.0

32
$-\quad 54$
$-\quad 56$

34

$19 \quad 2.27$

6.8

20

$-$

4.5

01105556 - NEPONSET RI VER NEAR DENHAM MASS (LAT 421233 LONG 0710847 )

OC.T. 1966

151

128

52

20

33

238

7.0

15 
TABLE 5.--CHEMICAL ANALYSES OF SURFACE WATER (CONTINUED)

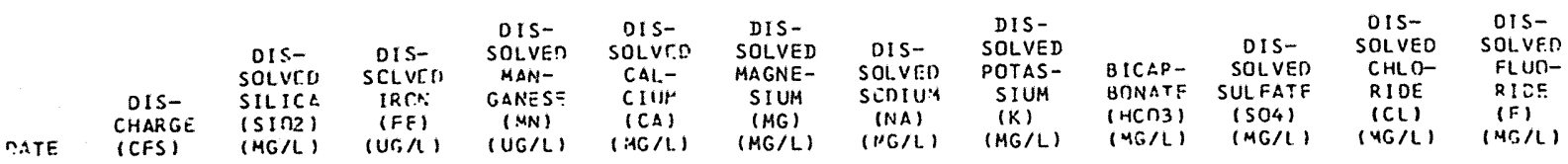

01105559 - NORROLAY RROOK AT NORTH RANDOLPH MASS (LAT 421104 LONG 0710308 )

$\begin{array}{cccccccccccc}\text { SEP. } & 1967 \\ 08 . . . & 0.35 & 9.8 & 430 & 110 & 15 & 4.2 & 29 & 3.2 & 36 & 25 & 46\end{array}$

01105561 - FARH RIVER NEAR BRAINTREE MASS (LAT 421230 LONG 0710227 )

$\begin{array}{ccccccccccccc}0.1 & 1966 \\ 07 . . . & 10 & 16 & 730 & 730 & 18 & 4.0 & 41 & 1.6 & 20 & 16 & 84 & .2\end{array}$
01105562 - FARM RIVER AT PONO STREET BRAINTREE MASS ILAT 421155 LONG 07101291

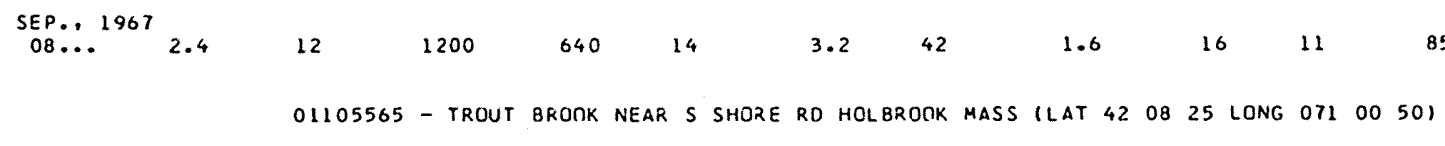

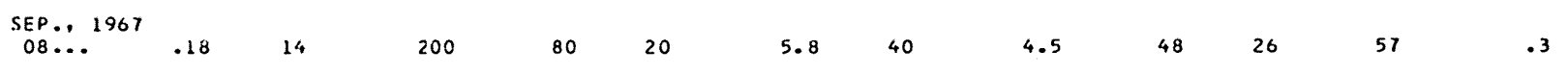

01105569 - MARY LEE BROOK AT RANDOLPH MASS (LAT 420939 LONG 0710144 )

SEP., 1967

$\begin{array}{ccccccccccc}08 . . . & .34 & 9.9 & 930 & 260 & 17 & 5.0 & 25 & 3.3 & 36 & 19\end{array}$

01105571 - GLOVE KS BROOK AT RANOOLPH MASS ILAT 421009 LONG 07102121

SEP., $1967 \quad .80$

$\begin{array}{llllllllll}14 & 1300 & 260 & 22 & 6.0 & 45 & 4.1 & 31 & 16 & 93\end{array}$

)

OCT. 1966

01105574 - COCHATO RIVER AT BRAINTRLE HIGHLANDS MASS (LAT 421049 LONG 0710104 )

$\begin{array}{llllllllll}9.0 & 730 & 250 & 13 & 4.9 & 21 & 3.2 & 24 & 27 & 39\end{array}$

01105575 - CRANBERRY HPOOK AT BRAINTREE HIGHLANDS MASS ILAT $4211 \quad$ U2 LOH 07100421

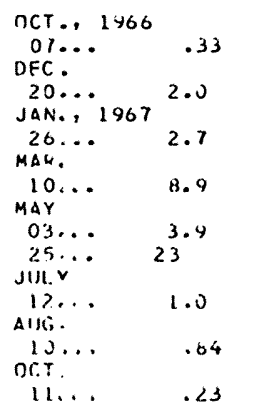

\begin{tabular}{|c|c|c|c|c|c|c|c|c|c|c|}
\hline 14 & 240 & 250 & 9.0 & 2.9 & 11 & .9 & 15 & 23 & 16 & .4 \\
\hline 10 & 230 & 250 & 11 & .3 & 9.4 & .8 & 7 & 20 & 15 & .4 \\
\hline H. 2 & 140 & 200 & 5.5 & 2.0 & 4.0 & .9 & 6 & 18 & 14 & .3 \\
\hline A. 0 & 140 & 200 & 5.2 & 1.9 & 9.0 & 1.0 & 4 & 10 & 14 & .3 \\
\hline $\begin{array}{l}3.4 \\
3.4\end{array}$ & $\begin{array}{r}420 \\
90\end{array}$ & $\begin{array}{l}2.80 \\
340\end{array}$ & $\begin{array}{l}8.0 \\
4.0\end{array}$ & $\begin{array}{l}1.7 \\
1.0\end{array}$ & $\begin{array}{l}4.7 \\
6.1\end{array}$ & $\begin{array}{l}1.1 \\
1.2\end{array}$ & $\begin{array}{l}7 \\
5\end{array}$ & $\begin{array}{l}20 \\
12\end{array}$ & 1.2 & $\begin{array}{r}.3 \\
.3\end{array}$ \\
\hline 4.6 & 100 & 350 & 7.1 & 2.6 & 11 & 1.6 & 14 & in & 10 & .4 \\
\hline 12 & 960 & $3 / C$ & 6.4 & 1.9 & 9.1 & 1.5 & 16 & 11 & 14 & .3 \\
\hline 14 & 1300 & 309 & 7.5 & $\therefore, t$ & 9.6 & $\therefore .4$ & 3 & 14 & 18 & 4 \\
\hline
\end{tabular}

O1105580 - MONATIQLOT RIVER AT SOUTH BRAINTREF MASS ILAT 421151 LONG O71 00311

\begin{tabular}{|c|c|c|c|c|c|c|c|c|c|c|c|c|}
\hline $\begin{array}{c}07 . . \\
\text { DEC. }\end{array}$ & 6.1 & 10 & 170 & 110 & 10 & 3.6 & 20 & 2.2 & 17 & 11 & 37 & .2 \\
\hline $\begin{array}{l}20 \ldots \\
\text { JAM.. }\end{array}$ & $67^{19}$ & 7.9 & 100 & 50 & 18 & 2.4 & 21 & 2.0 & 13 & 32 & 42 & .2 \\
\hline $\begin{array}{l}2 A . \\
\text { MAQ. }\end{array}$ & 38 & 5.5 & 70 & 70 & 10 & 3.2 & 24 & 1.6 & 3 & 25 & 44 & .1 \\
\hline $\begin{array}{l}05 \ldots \\
10 \ldots \\
\text { JUNE }\end{array}$ & $\begin{array}{r}53 \\
110\end{array}$ & $\begin{array}{r}.1 \\
6.4\end{array}$ & $\begin{array}{l}60 \\
90\end{array}$ & $\begin{array}{l}70 \\
60\end{array}$ & $\begin{array}{l}12 \\
11\end{array}$ & $\begin{array}{l}3.2 \\
3.4\end{array}$ & $\begin{array}{l}31 \\
32\end{array}$ & $\begin{array}{l}1.9 \\
2.0\end{array}$ & $\begin{array}{l}15 \\
11\end{array}$ & $\begin{array}{l}24 \\
26\end{array}$ & $\begin{array}{l}53 \\
57\end{array}$ & $\begin{array}{l}. ? \\
. ?\end{array}$ \\
\hline JULY & 37 & 2.0 & 520 & 1600 & 13 & 3.5 & 31 & $2 \cdot 8$ & 34 & 11 & 52 & .3 \\
\hline $\begin{array}{l}12 \ldots \\
\text { AUG. }\end{array}$ & 20 & 12 & 0 & 2900 & 15 & 4.0 & 28 & 2.0 & 46 & 2.0 & 52 & .3 \\
\hline $\begin{array}{l}10 . . \\
O C T\end{array}$ & 12 & 19 & 1700 & 1400 & 12 & 3.3 & 26 & 1.8 & 36 & 4.6 & 48 & .2 \\
\hline $11 \ldots$ & 7.7 & 14 & $1 \mathrm{CCO}$ & 510 & 13 & 3.5 & 27 & 2.9 & 30 & 16 & 50 & .2 \\
\hline
\end{tabular}


TABLE 5.--CHEMICAL ANALYSES OF SURFACE HATER (CONTINUED)

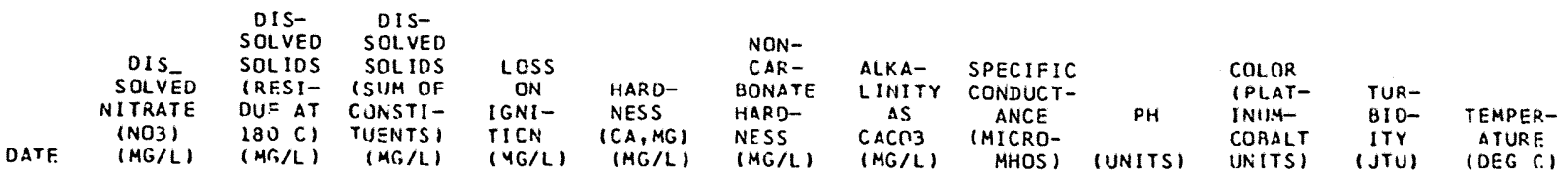

01105559 - NORROHAY BROOK AT NORTH RANDOLPH MASS ILAT 421104 LONG, 0710308 )

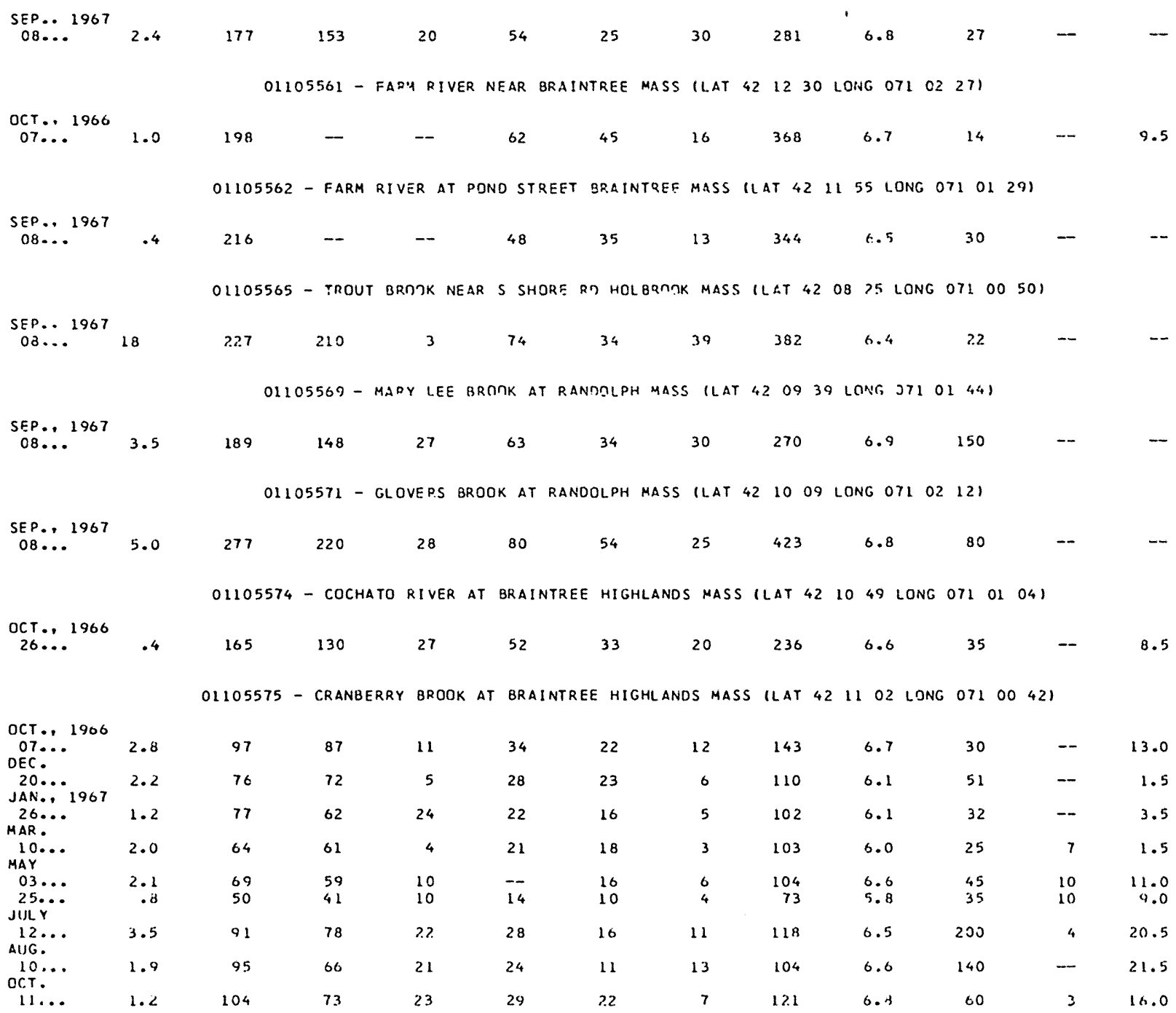

O1105580 - MINATIQLOT RIVER AT SUUTH BRAINTREF MASS ILAT 421151 LONG 07100311

\begin{tabular}{|c|c|c|c|c|c|c|c|c|c|c|c|c|}
\hline $\begin{array}{l}\text { OI... } \\
\text { DEC. }\end{array}$ & 13 & 132 & 115 & 20 & 40 & 26 & 14 & 203 & 6.6 & 35 & - & 10.5 \\
\hline $\begin{array}{l}20 \ldots .1967 \\
\text { JAN., } 1967\end{array}$ & .6 & 141 & 132 & 10 & 55 & 44 & 11 & .28 & 6.6 & 25 & -- & 1.0 \\
\hline $\begin{array}{l}26 \ldots \\
M \perp R\end{array}$ & .5 & 122 & 118 & -- & 38 & 32 & 7 & 224 & 6.3 & 14 & - & 1.0 \\
\hline $\begin{array}{l}05 \ldots \\
10 \ldots \\
\text { JUNF. }\end{array}$ & $\begin{array}{r}.3 \\
2.0\end{array}$ & $\begin{array}{l}148 \\
157\end{array}$ & $\begin{array}{l}133 \\
145\end{array}$ & 15 & $\begin{array}{l}43 \\
42\end{array}$ & $\begin{array}{l}31 \\
32 .\end{array}$ & $\begin{array}{r}12 \\
9\end{array}$ & $\begin{array}{l}261 \\
>75\end{array}$ & $\begin{array}{l}7.0 \\
6.5\end{array}$ & $\begin{array}{l}32 \\
15\end{array}$ & $\begin{array}{l}1 \\
2\end{array}$ & $\begin{array}{r}12.5 \\
1.0\end{array}$ \\
\hline $\begin{array}{l}08 \ldots . . \\
\text { JuLY }\end{array}$ & .9 & $15 \mathrm{C}$ & 134 & 12 & 47 & 19 & 28 & 257 & 6.5 & 79 & 3 & 18.5 \\
\hline $\begin{array}{l}12 \ldots \\
\text { AUS. }\end{array}$ & 2.4 & 159 & 141 & 17 & 54 & 16 & 38 & 257 & 7.0 & 100 & 3 & 21.0 \\
\hline OCT. & 1.0 & 157 & 134 & 21 & 44 & 14 & 30 & 230 & 6.9 & 80 & -- & 22.0 \\
\hline $11 \ldots$ & .6 & 162 & 142 & 21 & 47 & 22 & 25 & 243 & 7.1 & 37 & 3 & 15.5 \\
\hline
\end{tabular}


TABLE 5.--CHEMICAL ANALYSES OF SURFACE WATER (CONTINUED)

\begin{tabular}{|c|c|c|c|c|c|c|c|c|c|c|c|c|}
\hline$A^{T} E$ & $\begin{array}{l}\text { DIS- } \\
\text { CHARGE } \\
\text { (CFS) }\end{array}$ & $\begin{array}{l}\text { DIS- } \\
\text { SOLVED } \\
\text { SILICA } \\
\text { (SIOL) } \\
\text { (MG/L) }\end{array}$ & $\begin{array}{l}\text { OIS- } \\
\text { SOLVEn } \\
\text { IRCN } \\
\text { (FE) } \\
\text { (US/L) }\end{array}$ & $\begin{array}{l}\text { DIS- } \\
\text { SOLVED } \\
\text { MAN- } \\
\text { GANESF } \\
\text { (MN) } \\
\text { (UG/L) }\end{array}$ & $\begin{array}{l}\text { DIS- } \\
\text { SOLVED } \\
\text { CAL- } \\
\text { CIUM } \\
\text { (CA) } \\
\text { (MEIL) }\end{array}$ & $\begin{array}{l}\text { DIS- } \\
\text { SOLVED } \\
\text { MAGNE- } \\
\text { SIUM } \\
\text { (MG) } \\
\text { (MG/L) }\end{array}$ & $\begin{array}{l}\text { DIS- } \\
\text { SCLVER } \\
\text { SCDIUM } \\
\text { (NA) } \\
\text { (MG/L) }\end{array}$ & $\begin{array}{l}\text { DIS- } \\
\text { SOLVED } \\
\text { POTAS- } \\
\text { SIUM } \\
\text { (K) } \\
(M G / L)\end{array}$ & $\begin{array}{l}\text { BICIR- } \\
\text { BUN } \& \text { T } \\
(H C \cap 3) \\
(Y G / 1)\end{array}$ & $\begin{array}{l}\text { DIS- } \\
\text { SOLVEO } \\
\text { SUL FATE } \\
(S 1) 4) \\
(4 G / L)\end{array}$ & $\begin{array}{l}\text { OIS- } \\
\text { SOLVEO } \\
\text { CHLO- } \\
\text { RIDE } \\
\text { ICL) } \\
\text { (UE/LI }\end{array}$ & $\begin{array}{l}\text { DIS- } \\
\text { SOLVFO } \\
\text { FLUS- } \\
\text { RIIF } \\
\text { (F) }\end{array}$ \\
\hline
\end{tabular}

01105590 - MILL PIVER NEAR SOUTH WEYMOUTH MASS (LAT 421135 LONG 0705735 )

\begin{tabular}{|c|c|c|c|c|c|c|c|c|c|c|c|c|}
\hline $\begin{array}{l}\text { OCT.1 } 1966 \\
07 \ldots . .1967 \\
\text { SEP.. } 1967\end{array}$ & 0.70 & 13 & 1200 & 80 & 11 & 4.6 & 20 & 1.7 & 29 & 22 & 29 & 0. \\
\hline $08 \ldots$ & .40 & 12 & 520 & 80 & 11 & 4.7 & 18 & 2.1 & 38 & 17 & 26 & .3 \\
\hline
\end{tabular}

01105594 - CLD SWAMP RIVER AT FORREST ST ROCKLAND MASS (LAT 420859 LONG 0705510$)$

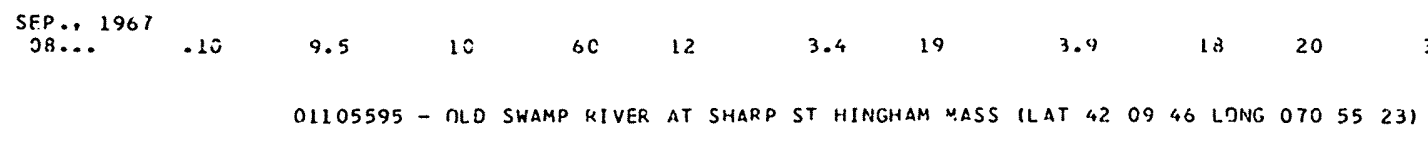

$\begin{array}{llllllllllllll}\text { SEP.. } 1967 & 1967 . . . & .30 & 14 & 360 & 44 & 7.0 & 2.2 & 9.8 & 1.4 & 73 & 3.4 & 16 & .2\end{array}$

01105598 - CLO SWAMO 2 AT PLHASANT ST S HEYMUUTH MASS ILAT 4211 i3 LONG 2705602.011

\begin{tabular}{|c|c|c|c|c|c|c|c|c|c|c|c|c|}
\hline $\begin{array}{l}28 \ldots \\
\text { AUS... }\end{array}$ & -- & -- & -- & -- & -- & -- & -- & -- & 15 & 5.6 & 3.0 & - \\
\hline SFP... & -- & -- & - & -- & $-\infty$ & - & -- & -- & 17 & 11 & 14 & $\cdots$ \\
\hline 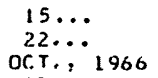 & $\begin{array}{l}.05 \\
.44\end{array}$ & -- & -- & $=$ & - & - & -- & $\cdots$ & $\begin{array}{l}40 \\
25\end{array}$ & $\begin{array}{l}13 \\
15\end{array}$ & $\begin{array}{l}11 \\
11\end{array}$ & $\overline{-}$ \\
\hline SFP.: 1967 & 2.0 & 11 & 240 & 30 & 8.5 & 3.3 & 22 & 1.3 & 16 & 20 & 37 & .3 \\
\hline $6: 8, \ldots$ & 1.4 & 12. & 150 & 30 & 8.3 & 3.3 & $\angle 2$ & 1.6 & $\therefore 1$ & 13 & 38 & .2 \\
\hline
\end{tabular}

01105600 - OLO SWGMP RIVER NEAR SOUTH WIYMCUTH MASS ILAT 421125 I.ONG 07056431

\begin{tabular}{|c|c|c|c|c|c|c|c|c|c|c|c|c|}
\hline $\begin{array}{r}\text { DFC.: } 1966 \\
20 \ldots \ldots \\
\text { JA: } 1967\end{array}$ & 6.2 & 10 & 200 & 320 & 14 & 1.1 & 21 & 1.3 & 3 & 24 & $3 t$ & $\cdot 2$ \\
\hline $\begin{array}{l}26 \ldots \ldots \\
N \Delta E .\end{array}$ & 5.5 & 9.3 & 110 & 110 & 3.0 & 2.8 & 22 & i. 3 & 10 & 21 & 35 & .1 \\
\hline $\operatorname{m.1Y}$ & 24 & 7.6 & 120 & 120 & 7.1 & 2.6 & $? 2$ & 1.3 & $\mathrm{~B}$ & 20 & 37 & .2 \\
\hline $\begin{array}{l}21 \ldots \\
22 \ldots \\
\text { Jui } . .\end{array}$ & $\begin{array}{l}7 \cdot 3 \\
6.2\end{array}$ & $\begin{array}{l}5.3 \\
5.5\end{array}$ & $\begin{array}{r}123 \\
90\end{array}$ & $\begin{array}{l}129 \\
100\end{array}$ & $\begin{array}{l}3 . ? \\
7.6\end{array}$ & $\begin{array}{l}2.9 \\
2.5\end{array}$ & $\begin{array}{l}23 \\
22\end{array}$ & $\begin{array}{l}1.5 \\
1.6\end{array}$ & $\begin{array}{l}12 \\
14\end{array}$ & $\begin{array}{l}21 \\
16\end{array}$ & $\begin{array}{l}37 \\
13\end{array}$ & $\begin{array}{l}.2 \\
.2\end{array}$ \\
\hline $\begin{array}{l}12 \ldots \\
\text { Duj: }\end{array}$ & $2 . B$ & 10 & 3) & $: 31$ & 9.2 & 3.3 & 23 & 1.7 & $2 \%$ & 13 & $? 8$ & .2 \\
\hline $\begin{array}{l}10 \ldots \\
\text { ors. }\end{array}$ & 2.3 & 9.3 & 260 & 120 & 8.4 & 2.6 & 18 & 1.6 & 23 & 10 & 30 & $\cdot 2$ \\
\hline $11 \cdots$ & 1.1 & 14 & 330 & 50 & 13 & $3 \cdot 3$ & 24 & 1.9 & 18 & 25 & 40 & .2 \\
\hline
\end{tabular}

01105610 - WHITMANS POND OUTLET AT EAST WEYMOUTH MASS (LAT 421245 LONG O70 5532 )

OCT. 1966
$10 \ldots . .1967$
SEP. 1907
$08 \ldots$

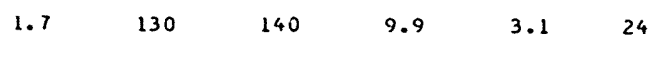

$\begin{array}{llll}1.9 & 22 & 19 & 38\end{array}$

$\begin{array}{lllllllllll}1.4 & 580 & 120 & 7.7 & 2.6 & 18 & 1.9 & 20 & 13 & 28\end{array}$

.2

01105617 - FRESH RIVER NEAR HINGHAM MASS (LAT 421330 LONG 0705453 )

\begin{tabular}{|c|c|c|c|c|c|c|c|c|c|c|c|c|}
\hline $\begin{array}{l}10 \ldots \ldots 1967 \\
\text { SEP.. } 196\end{array}$ & .54 & 11 & 580 & 100 & 12 & 3.8 & 15 & 1.8 & 32 & 19 & 24 & .2 \\
\hline $08 \ldots$ & .61 & 11 & 150 & 250 & 13 & 3.6 & 17 & 1.4 & 37 & 13 & 34 & .2 \\
\hline
\end{tabular}


TABLE 5.--CHEMICAL ANALYSES OF SURfACE WATER (CONTINUED)

\begin{tabular}{|c|c|c|c|c|c|c|c|c|c|c|c|c|}
\hline DATE & $\begin{array}{l}\text { DIS- } \\
\text { SOLVED } \\
\text { NITRATE } \\
\text { (NO3) } \\
\text { (MG/L) }\end{array}$ & $\begin{array}{l}\text { DIS- } \\
\text { SOLVEC } \\
\text { SOLIDS } \\
\text { (RESI- } \\
\text { DUF AT } \\
180 . C \text { C) } \\
\text { (MG/I) }\end{array}$ & $\begin{array}{l}\text { DIS- } \\
\text { SOLVED } \\
\text { SOLIDS } \\
\text { (SUM OF } \\
\text { CONSTI- } \\
\text { TUENTS) } \\
\text { (MGIL) }\end{array}$ & $\begin{array}{l}\text { LOSS } \\
\text { CN } \\
\text { IGNI- } \\
\text { TION } \\
\text { (MGIL) }\end{array}$ & $\begin{array}{l}\text { HARD- } \\
\text { NESS } \\
\text { (CA,MG) } \\
\text { (MG/L) }\end{array}$ & $\begin{array}{l}\text { NON- } \\
\text { CAR- } \\
\text { BONATE } \\
\text { HARD- } \\
\text { NESS } \\
\text { (MG/L) }\end{array}$ & $\begin{array}{l}\text { ALKA- } \\
\text { LINITY } \\
\text { AS } \\
\text { CACO3 } \\
(M G / L)\end{array}$ & $\begin{array}{c}\text { SPECIFIC } \\
\text { CONDUCT- } \\
\text { ANCE } \\
\text { (MICRO- } \\
\text { MHOS) }\end{array}$ & $\begin{array}{c}P H \\
\text { (UNITS) }\end{array}$ & $\begin{array}{l}\text { COLOR } \\
\text { (PLAT- } \\
\text { INUM- } \\
\text { COBALT } \\
\text { UN ITS) }\end{array}$ & $\begin{array}{l}\text { TUR- } \\
\text { BID- } \\
\text { ITY } \\
\text { ITUI }\end{array}$ & $\begin{array}{l}\text { TEMPER- } \\
\text { ATURE } \\
\text { (DEG C) }\end{array}$ \\
\hline
\end{tabular}

01105590 - MILL RIVER NEAR SOUTH WEYMOUTH MASS (LAT 421135 LONG 0705735 )

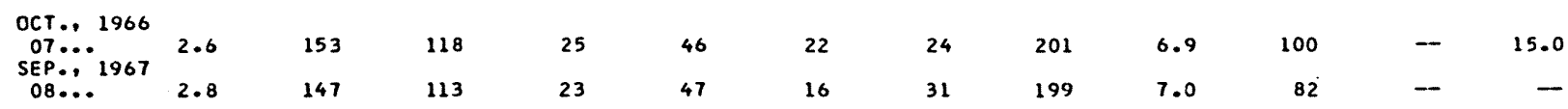

01105594 - CLD SWAMP RIVER AT FORREST ST ROCKLAND MASS (LAT 420859 LONG OTO 5510$)$

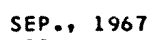

44

29

15

221

01105595 - OLD SWAMP RIVER AT SHARP ST HINGHAM MASS (LAT 420946 LONG 07055231

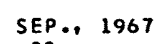

01105598 - CLD SHAMP R AT PLEASANT ST S WEYMDUTH MASS (LAT 421113 LONF 0705602.01$)$

\begin{tabular}{|c|c|c|c|c|c|c|c|c|c|c|c|c|}
\hline $\begin{array}{l}\text { JULY, } 1959 \\
28 \ldots . . \\
\text { AUG. }\end{array}$ & 3.8 & -- & -- & -- & 28 & 16 & 12 & 77 & 6.0 & 300 & -- & 19.5 \\
\hline $\begin{array}{l}17 \ldots \\
\text { SEP. }\end{array}$ & 5.8 & -- & -- & -- & 34 & 20 & 14 & 100 & 6.5 & 180 & - & 23.5 \\
\hline $\begin{array}{l}15 \ldots \\
22 \ldots \ldots \\
\text { OCT. } 1966\end{array}$ & $\begin{array}{l}4.1 \\
5.1\end{array}$ & -- & - & - & $\begin{array}{l}42 \\
38\end{array}$ & $\begin{array}{r}9 \\
15\end{array}$ & $\begin{array}{l}33 \\
21\end{array}$ & $\begin{array}{l}138 \\
125\end{array}$ & $\begin{array}{l}0.7 \\
6.5\end{array}$ & $\begin{array}{l}100 \\
100\end{array}$ & - & $\begin{array}{l}13.5 \\
19.0\end{array}$ \\
\hline $\begin{array}{l}07 \ldots . . \\
\text { SEP., } 1967\end{array}$ & 1.8 & 155 & 108 & 26 & 34 & 22 & 13 & 189 & 5.4 & 95 & -- & 11.5 \\
\hline $08 \ldots$ & 2.6 & 147 & 111 & 12 & 34 & 17 & 17 & 2.01 & 6.8 & 130 & -- & - \\
\hline
\end{tabular}

01105600 - OLD SHAMP RIVER NEAR SOUTH WFYMOUTH MASS (LAT 421125 LONG 070 S6 43 )

\begin{tabular}{|c|c|c|c|c|c|c|c|c|c|c|c|c|}
\hline $20 \ldots 1967$ & $2 \cdot 1$ & 127 & 114 & 11 & 40 & 32 & 7 & 144 & 3.2 & 60 & -- & 2.0 \\
\hline $\begin{array}{c}26 \cdots \\
\text { MAE. }\end{array}$ & 2.2 & 122 & 107 & 19 & 32 & 24. & 8 & 190 & 5.3 & 35 & - & 5.0 \\
\hline$\underset{\text { MOY }}{04 . .}$ & 2.5 & 122 & 104 & 26 & 28 & 2.2 & 7 & 192 & 0.3 & 45 & 30 & 3.0 \\
\hline $\begin{array}{l}01 \ldots \\
22 \ldots \ldots \\
\text { JII.Y }\end{array}$ & $\begin{array}{l}2.0 \\
1.4\end{array}$ & $\begin{array}{l}111 \\
11\end{array}$ & $\begin{array}{r}107 \\
47\end{array}$ & $\begin{array}{l}13 \\
2.1\end{array}$ & $\begin{array}{l}32 \\
29\end{array}$ & $\begin{array}{l}22 \\
18\end{array}$ & $\begin{array}{l}10 \\
11\end{array}$ & $\begin{array}{l}194 \\
182\end{array}$ & $\begin{array}{l}7.1 \\
6.9\end{array}$ & $\begin{array}{l}55 \\
92\end{array}$ & - & $\begin{array}{l}10.5 \\
14.0\end{array}$ \\
\hline $\begin{array}{l}12 \ldots \\
\text { AUG. }\end{array}$ & 2.9 & 126 & 113 & 19 & 36 & 17 & 20 & 199 & 6.9 & 110 & 2 & 18.5 \\
\hline OCT. & 1.6 & 110 & 93 & 20 & 32 & 12 & 19 & 163 & 6.8 & 120 & - & 20.5 \\
\hline $11 \ldots$ & 1.6 & 152 & 132 & 24 & 46 & 31 & 15 & 213 & 6.3 & 120 & 1 & 14.0 \\
\hline
\end{tabular}

01105610 - WHITMANS POND OUTLET AT EAST HEYMOUTH MASS (LAT 421245 LONG 0705532 )

\begin{tabular}{|c|c|c|c|c|c|c|c|c|c|c|c|c|}
\hline SEP. 1967 & 1.9 & 151 & 111 & 39 & 38 & 20 & 18 & 212 & & 24 & - & \\
\hline $08 \ldots$ & 1.1 & 104 & 84 & 15 & 30 & 13 & 16 & 166 & 6.6 & 60 & -- & -- \\
\hline
\end{tabular}
01105617 - FRESH RIVER NEAR HINGHAM MASS ILAT $4213 \quad 30$ LONG 0705453 )

\begin{tabular}{|c|c|c|c|c|c|c|c|c|c|c|c|c|}
\hline $\begin{array}{l}\text { OCT.. } 1966 \\
10 \ldots \ldots 1967 \\
\text { SEP.. } 1967\end{array}$ & .2 & 124 & 103 & 14 & 46 & 20 & 26 & 181 & 6.9 & 20 & -- & 14.0 \\
\hline $08 \ldots$ & .1 & 131 & 111 & -- & 48 & 17 & 30 & 209 & 7.2 & 15 & - & -- \\
\hline
\end{tabular}


Table 6.--List of basic-data reports for

Massachusetts and New Hampshire ${ }^{1}$

\section{MASSACHUSETTS}

*I Wilmington-Reading Area, by John A. Baker and Edward A. Sammel, 1961, 2 figs. Covers an area of about 43 square miles in the upper part of the Ipswich River basin in northeastern Massachusetts.

*2 Lower Ipswich River basin, by Edward A. Sammel and John A. Baker, 1962, 47 p., 2 figs. Covers an area of about 110 square miles in northeastern Massachusetts.

3 Lowell Area, by John A. Baker and Richard G. Petersen, 1962, 28 p., 2 figs. Covers an area of about 115 square miles and includes most of the metropolitan area of the city of Lowell.

*4 Parker and Rowley River basins, by Edward A. Sammel, 1962, 33 p., 2 figs. The rivers drain an area of about 77 square miles in northeastern Massachusetts.

*5 Brockton-Pembroke Area, by Richard G. Petersen, 1962, 46 p., 2 figs. Covers an area of about 112 square miles in the northern part of Plymouth County.

*6 Western Massachusetts, by Richard G. Petersen and Anthony Maevsky, 1962, 31 p., I fig. Covers an area of about 2,865 square miles and includes all of Berkshire, Franklin, Hampshire, and Hampden Counties.

*7 Southeastern Massachusetts, by Anthony Maevsky and Janet A. Drake, 1963, 55 p., 2 figs. Covers an area of about 1,930 square miles and includes all of Barnstable, Bristol, Dukes, Nantucket, and Plymouth Counties (exclusive of the Brockton-Pembroke Area).

8 Assabet River basin, by Samuel J. Pollock and William B. Fleck, 1964, $45 \mathrm{p} ., 1 \mathrm{pl}$. Covers an area of approximately 177 square miles and includes parts of Middlesex and Worcester Counties.

*9 Housatonic River basin, by Ralph F. Norvitch and Mary E.S. Lamb, 1966, 50 p., I pl. Covers an area of about 530 square miles in the upper part of the basin, which is north of the Connecticut-Massachusetts state line.

10 Northern part, Ten Mile and Taunton River basins, by John R. Williams and Richard E. Willey, 1967, 56 p., I pl., I fig. Covers an area of about 195 square miles within Bristol, Norfolk, and Plymouth Counties.

11 Millers River basin, by Donald R. Wiesnet and William B. Fleck, 1967, 29 p., 1 pl., 1 fig. Covers an area of about 392 square miles within Franklin and Worcester Counties, Massachusetts, and Hillsborough and Cheshire Counties, New Hampshire. 
Table 6.--List of basic-data reports for

Massachusetts and New Hampshire (Continued)

MASSACHUSETTS (Continued)

12 Taunton River basin, by John R. Williams and Richard E. Willey, 1970, 102 p., 1 pl., 1 fig. Covers an area of about 528 square miles in Bristol, Norfolk, and Plymouth Counties, Massachusetts.

13 Deerfield River basin, by Bruce P. Hansen, Frederick B. Gay, and L.G. Toler, 1973, 59 p., I fig., I pl. Covers an area of 348 square miles in northwestern Massachusetts.

\section{NEW HAMPSHIRE}

*I Southeastern Area, by Edward Bradley and Richard G. Petersen, 1962, 53 p., 5 figs. Covers an area of about 390 square miles in parts of Rockingham and Strafford Counties.

2 Lower Merrimack River valley, by James M. Weigle and Richard Kranes, 1966, 44 p., 1 pl. Covers an area of about 396 square miles in centralsouthern New Hampshire.

3 Ashuelot River basin, by Harold A. Whitcomb, 1973, 25 p., 1 pl. Covers an area of about 420 square miles in southwestern New Hampshire.

IThese reports are available, free of charge, at the U.S. Geological Survey, 150 Causeway Street, Boston, MA 02114. An asterisk indicates that the report is out of print but may be consulted at the above office and at many public and educational institution libraries. 